\title{
How efficient deep-learning object detectors are?
}

\author{
Luis Miguel Soria ${ }^{\text {a, }}$ *, Francisco J Ortega ${ }^{\text {b }}$, Juan A. Álvarez-García a, Francisco Velasco b ${ }^{\text {, Damián }}$ \\ Fernández-Cerero ${ }^{a}$
}

a Departamento de Lenguajes y Sistemas Informãticos, Universidad de Sevilla, Spain

${ }^{\mathrm{b}}$ Departamento de EconomÃa Aplicada I, Universidad de Sevilla, Spain

Keywords:

Neural networks

Deep learning

Object detection Efficiency

analysis

Data envelopment

analysis

\begin{abstract}
A B S T R A C T
Deep-learning object-detection architectures are gaining attraction, as they are used for critical tasks in relevant environments such as health, self-driving, industry, security, and robots. Notwithstanding, the available architectures provide variable performance results depending on the scenario under consideration. Challenges are usually used to evaluate such performance only in terms of accuracy. In this work, instead of proposing a new architecture, we overcome the limitations of those challenges by proposing a computationally undemanding comparative model based on several Data Envelopment Analysis (DEA) strategies, not only for the comparison of deep-learning architectures, but also to detect which parameters are the most relevant features for achieving efficiency. In addition, the proposed model provides with a set of recommendations to improve object-detection frameworks. Those measures may be applied in future high-performance meta-architectures, since this model requires lower computational and temporal requirements compared to the traditional strategy based on training neural networks - based on the trial-error method - for each configurable parameter. To this aim, the presented model evaluates 16

parameters of 139 configurations of well-known detectors present in the Google data set [1].
\end{abstract}

\section{Introduction}

Object detection is a research area that is attracting interest in recent years. Deep-learning architectures are being used in diverse object-detection contexts [2], such as: (a) computer vision $[3,4]$; (b) robots [5]; and (c) health [6]. The performance of the deep-learning architectures on each context is sometimes hard to evaluate, being such analysis crucial to determine which architecture is the best suited for each context.

Moreover, new architectures, feature extractors, and combinations of both of them are continuously presented. A fair comparison between different object detectors is also difficult to carry out due to the high number of characteristics under analysis. Such architectures and detectors are commonly evaluated by challenges, which consider common objects, such as pedestrians, and vehicles. These challenges include: Imagenet [7], PASCAL VOC [8], and Microsoft COCO [9]. In these challenges, only speed and accuracy are considered. Therefore, several other relevant metrics may be left out of the evaluation process.

\footnotetext{
* Corresponding author.

E-mail addresses: Isoria@us.es (L.M. Soria), fjortega@us.es (F.J. Ortega), jaalvarez@us.es (J.A. Álvarez-García), velasco@us.es (F. Velasco), damiancerero@us.es (D. Fernández-Cerero).
}

In this paper, we overcome this limitation by employing Data Envelopment Analysis (DEA) for the comparison of several wellknown detectors based on SSD, R-CNN, and R-FCN. Since DEA is claimed to lack homogeneity between different models, we employ three different DEA models to improve the robustness and flexibility of the analysis performed: (a) the original DEA model (managerial); (b) a DEA model based on probabilistic techniques; and (c) a DEA model based on Bayesian techniques. The main aims of the experimentation performed are: (a) to provide a formal framework to determine which combinations of detectors, as well as their related input and output parameters, are efficient (related to performance in this work); (b) to empirically detect which parameters affect the most to the performance of object-detection frameworks; (c) to provide a model able to formally propose a set of in-detail corrections to improve each parameter of the analysed object detection models.

The main contributions of this work include:

1. A model for the efficiency analysis of different neural-network configurations.

2. A formal framework for the determination of the most crucial parameters for object detection performance; and

3. A simple and low-computing-demanding methodology to improve the efficiency for deep-learning architectures. 
Table 1

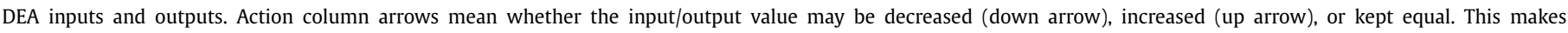
reference to positive and negative inputs/outputs.

\begin{tabular}{|c|c|c|}
\hline Parameter & Description & Action \\
\hline \multicolumn{3}{|l|}{ Inputs } \\
\hline Image resolution & Size of the input images of the training set & $\uparrow \leftrightarrow$ \\
\hline Proposals sent to box classifier & Number of regions of the original image sent to the detection classifier & $\uparrow \leftrightarrow$ \\
\hline \multicolumn{3}{|l|}{ Outputs } \\
\hline $\mathrm{mAP}$ & Global mAP (accuracy) for the whole set of images in the validation set & $\uparrow \leftrightarrow$ \\
\hline mAP (small) & mAP for small-sized region of interest & $\uparrow \leftrightarrow$ \\
\hline mAP (medium) & mAP for medium-sized region of interest & $\uparrow \leftrightarrow$ \\
\hline mAP (large) & mAP for large-sized region of interest & $\uparrow \leftrightarrow$ \\
\hline mAP@. 75IOU & mAP with an $75 \%$ overlapping threshold between ground truth and bounding box & $\uparrow \leftrightarrow$ \\
\hline mAP @. 50IOU & mAP with an $50 \%$ overlapping threshold between ground truth and bounding box & $\uparrow \leftrightarrow$ \\
\hline Memory & RAM memory consumed during learning process $(\mathrm{MiB})$ & $\downarrow \leftrightarrow$ \\
\hline Memory std & Average deviation of memory consumption (MiB) & $\downarrow \leftrightarrow$ \\
\hline CPU & CPU time consumed by each batch (milliseconds) & $\downarrow \leftrightarrow$ \\
\hline CPU std & Time deviation of CPU consumption (milliseconds) & $\downarrow \leftrightarrow$ \\
\hline GPU std & Time and deviation of GPU utilisation & $\downarrow \leftrightarrow$ \\
\hline FLOPS & Floating operations during training & $\downarrow \leftrightarrow$ \\
\hline
\end{tabular}

Table 2

Sample of the data set used for DEA analysis.

\begin{tabular}{|c|c|c|c|c|c|c|c|c|c|}
\hline \# & Architecture, extractor & Resolution & mAP & mAP@. 75IOU & \# params & Memory & CPU & GPU & FLOPS \\
\hline 1 & $\begin{array}{l}\text { Faster RCNN, } \\
\text { VGG }\end{array}$ & 300 & 22.90 & 22.90 & 138.51 & 1699.00 & 27.00 & 16.00 & 64.32 \\
\hline 2 & $\begin{array}{l}\text { Faster RCNN, } \\
\text { VGG }\end{array}$ & 300 & 19.60 & 20.40 & 138.51 & 1703.00 & 41.00 & 16.00 & 29.12 \\
\hline 11 & $\begin{array}{l}\text { Faster RCNN, } \\
\text { Resnet } 101\end{array}$ & 300 & 26.50 & 27.20 & 63.15 & 3236.00 & 28.00 & 14.00 & 239.42 \\
\hline 18 & $\begin{array}{l}\text { Faster RCNN, } \\
\text { Resnet } 101\end{array}$ & 600 & 29.40 & 31.80 & 63.15 & 1985.00 & 93.00 & 16.00 & 91.35 \\
\hline 31 & $\begin{array}{l}\text { Faster RCNN, } \\
\text { Inception V2 }\end{array}$ & 300 & 15.40 & 14.80 & 13.31 & 2370.00 & 5.00 & 15.00 & 118.22 \\
\hline 32 & $\begin{array}{l}\text { Faster RCNN, } \\
\text { Inception V2 }\end{array}$ & 300 & 13.30 & 13.40 & 13.31 & 211.00 & 7.00 & 13.00 & 7.63 \\
\hline 49 & $\begin{array}{l}\text { Faster RCNN, } \\
\text { Inception V3 }\end{array}$ & 600 & 28.60 & 29.90 & 26.27 & 1522.00 & 60.00 & 16.00 & 74.37 \\
\hline 50 & $\begin{array}{l}\text { Faster RCNN, } \\
\text { Inception V3 }\end{array}$ & 600 & 29.20 & 30.20 & 26.27 & 2293.00 & 5.00 & 13.00 & 124.51 \\
\hline 51 & $\begin{array}{l}\text { Faster RCNN, } \\
\text { Inception Resnet V2 }\end{array}$ & 300 & 28.40 & 29.30 & 60.02 & 14769.00 & 104.00 & 15.00 & 639.98 \\
\hline 70 & $\begin{array}{l}\text { Faster RCNN, } \\
\text { Inception Resnet V2 }\end{array}$ & 600 & 35.30 & 37.60 & 60.02 & 10341.00 & 38.00 & 14.00 & 370.69 \\
\hline 71 & $\begin{array}{l}\text { Faster RCNN, } \\
\text { MobileNet }\end{array}$ & 300 & 16.40 & 15.50 & 6.06 & 1147.00 & 22.00 & 17.00 & 25.23 \\
\hline 72 & $\begin{array}{l}\text { Faster RCNN, } \\
\text { MobileNet }\end{array}$ & 300 & 14.40 & 14.20 & 6.06 & 143.00 & 5.00 & 7.00 & 2.55 \\
\hline 81 & $\begin{array}{l}\text { R-FCN, } \\
\text { Resnet } 101\end{array}$ & 300 & 25.20 & 25.90 & 68.48 & 992.00 & 15.00 & 6.00 & 27.14 \\
\hline 82 & $\begin{array}{l}\text { R-FCN, } \\
\text { Resnet } 101\end{array}$ & 300 & 25.20 & 26.00 & 68.48 & 861.00 & 25.00 & 5.00 & 27.14 \\
\hline 101 & $\begin{array}{l}\text { R-FCN, } \\
\text { Inception V2 }\end{array}$ & 300 & 15.40 & 14.50 & 18.06 & 286.00 & 7.00 & 5.00 & 5.09 \\
\hline 102 & $\begin{array}{l}\text { R-FCN, } \\
\text { Inception V2 }\end{array}$ & 300 & 15.50 & 14.60 & 18.06 & 208.00 & 6.00 & 6.00 & 5.09 \\
\hline 111 & $\begin{array}{l}\text { R-FCN, } \\
\text { Inception Resnet V2 }\end{array}$ & 300 & 22.50 & 22.70 & 65.06 & 696.00 & 10.00 & 6.00 & 14.99 \\
\hline 112 & $\begin{array}{l}\text { R-FCN, } \\
\text { Inception Resnet V2 }\end{array}$ & 300 & 22.80 & 23.00 & 65.06 & 642.00 & 13.00 & 7.00 & 14.99 \\
\hline 131 & $\begin{array}{l}\text { R-FCN, } \\
\text { MobileNet }\end{array}$ & 300 & 15.00 & 13.70 & 10.80 & 246.00 & 4.00 & 5.00 & 2.44 \\
\hline 132 & $\begin{array}{l}\text { R-FCN, } \\
\text { MobileNet }\end{array}$ & 300 & 15.20 & 13.80 & 10.80 & 170.00 & 8.00 & 7.00 & 2.44 \\
\hline
\end{tabular}

The rest of this paper is organised as follows. In Section 2, we briefly introduce DEA and object detectors. A formal definition of the employed DEA models is provided in Section 3. The analysis performed and obtained results are outlined in Section 4. Finally, in Section 5, we provide the conclusions and discuss the future work.

\section{State of the art}

In this section, we discuss the literature regarding DEA and object detection.

\subsection{Data envelopment analysis}

DEA was suggested in [10] to quantify productive efficiency of decision-making units (DMU). This non-parametric method assesses efficiency through the analysis of a set of inputs and outputs. DEA has been proved useful to empirically compute the efficiency of DMUs and to determine whether such DMUs belong or not to the "frontier" of production. In this context, the frontier of production, that is, the efficiency frontier, is composed of the most efficient DMUs, and is utilised to compute the relative 
efficiency of the rest of DMUs. DEA is a practical tool when diverse inputs and outputs with unknown relationships between them are under consideration. Although DEA was initially employed only in operations research and economic environments, several applications of DEA have emerged for the measurement of the efficiency in diverse areas, such as:

(a) energies [11,12]; (b) decision-support systems [13,14]; (c) research evaluation [15-17]; (d) public sector analysis [18-20]; (e) entrepreneurship [21]; (f) environment [22,23]; and (g) agriculture [24-26].

One of the main advantages of DEA is that it sets up a bestpractice production frontier that may be used to decide the level of inefficiency of DMUs. There are several possible DEA modes:

- The original approach proposed in [10] is input-oriented. Therefore, the desired output level is obtained by minimising the input production, and assumes constant returns to scale (CRS): the increment of inputs lead to an increase of outputs.

- Another extended model, proposed in [27], is output-oriented. In this case, DEA tries to maximise outputs with a set of fixed available inputs.

- Finally, the model proposed in [28] employs variable returns to scale (VRS). In this model, an increment of inputs may induce a change in the amount of outputs generated [29].

\subsection{Object detectors}

Detection architectures are constantly evolving, improving metrics for the detection of various kind of images, such as: (a) faces [30]; (b) handguns [31]; and (c) traffic signs [32]. State-of-the-art object-detection algorithms, such as R-CNN, R-FCN, SSD, and YOLO [33-36], use deep-neural-network architectures. There are several metrics (outputs) to take into account to correctly measure the efficiency of each algorithm in various situations, including: (a) mean average precision (mAP); (b) inference times; and (c) memory consumption. Such measurement may help to decide which detector suits best a particular application. For instance, mobile devices require lightweight-model architectures with low memory usage, whilst autonomous vehicles require good detection accuracy and real-time performance.

These detectors have been evaluated in several object-detection competitions, such as: (a) Imagenet [7]; (b) PASCAL VOC [8]; and (c) Microsoft COCO [9]. There exist comparisons of these detectors in specific contexts [37]. Notwithstanding, the long computation time needed to train them with several configurations (inputs parameters) may prevent the vast majority of users from evaluating them, leaving large companies, such as Google, as the only actor to analyse them [1]. As a result of such analysis, meta-architectures of Faster R-CNN, R-FCN, and SSD have been proposed, and have been combined with various feature extractors, such as: (a) VGG [38]; (b) Resnet 101 [39]; (c) Inception V2 [40]; (d) Inception V3 [41]; (e) Inception Resnet V2 [42]; and (f) Mobile Net [43], in order to compare a large number of detection systems in a unified manner.

In this work, we extend the state of the art by proposing a formal model for efficiency evaluation of deep-learning architectures, as well as providing a systematic framework for the improvement of efficiency of deep-learning architectures based on several relevant parameters.

\section{DEA models}

DEA assumes data to be free of measurement errors and is sensitive to outliers. Numerous works that introduce stochastic variation in production relationships and provide statistical inference in DEA contexts have been proposed recently [44]. In this work, we employ first the chance-constrained programming technique $[45,46]$. Assuming that the inputs and the outputs observed are drawn from a joint probability distribution which parameters are unknown, this approach allows exceptional cases with constraint violation (concretely, with a probability lesser than a fixed value $\alpha$ ). In a second stage, we take prior distributions for the unknown parameters, and we apply Bayesian Inference to obtain statistical measurements of DMUs efficiency.

Appendix A shows a detailed explanation of the mathematical development for each of the three DEA models used in this paper: managerial, probabilistic and Bayesian.

\section{DEA analysis}

In this Section, we present the structure of the data set used to carry out the comparison of the different architectures of neural networks, as well as the result of the process of applying the DEA models on the data set described. The analysis has been carried out by means of three different DEA models in order to test whether the conclusions provided by them are homogeneous or not, as well as the different nuances of each DEA model.

\subsection{Data set}

In this work, we employ the data set generated in [1], which includes the data and parameters for each detector, summing up 139 different configurations. It should be borne in mind that we do not aim to deeply describe the characteristics of each detector under comparison ([1] does), but rather to perform a statistical multi-criteria DEA analysis for the determination of the relative efficiency of a deep learning system in comparison with other existing systems. This data set is composed by the

Table 3

Summary of the result of the managerial DEA analysis (efficiency coefficients) grouped by image resolution for each of the analysed architectures.

\begin{tabular}{|c|c|c|c|}
\hline $\begin{array}{l}\text { Input image } \\
\text { resolution }\end{array}$ & $\begin{array}{l}\text { Average DEA } \\
\text { coefficient }\end{array}$ & $\begin{array}{l}\text { Min DEA } \\
\text { coefficient }\end{array}$ & $\begin{array}{l}\text { Max DEA } \\
\text { coefficient }\end{array}$ \\
\hline \multicolumn{4}{|c|}{$\begin{array}{l}\text { Faster RCNN } \\
\text { Inception Resnet V2 }\end{array}$} \\
\hline 300 & 0.7229 & 0.5841 & 1 \\
\hline 600 & 0.8203 & 0.6640 & 0.9926 \\
\hline \multicolumn{4}{|l|}{ Inception V2 } \\
\hline 300 & 0.8261 & 0.7094 & 0.9525 \\
\hline 600 & 0.8692 & 0.6974 & 1 \\
\hline \multicolumn{4}{|l|}{ Inception V3 } \\
\hline 300 & 0.7991 & 0.6229 & 1 \\
\hline 600 & 0.8852 & 0.6280 & 1 \\
\hline \multicolumn{4}{|l|}{ MobileNet } \\
\hline 300 & 0.9507 & 0.8768 & 1 \\
\hline 600 & 1 & 1 & 1 \\
\hline \multicolumn{4}{|l|}{ Resnet 101} \\
\hline 300 & 0.9317 & 0.6910 & 1 \\
\hline 600 & 0.9191 & 0.7925 & 1 \\
\hline \multicolumn{4}{|l|}{ VGG } \\
\hline 300 & 0.9975 & 0.9929 & 1 \\
\hline 600 & 0.9873 & 0.9367 & 1 \\
\hline \multicolumn{4}{|l|}{ R-FCN } \\
\hline \multicolumn{4}{|c|}{ Inception Resnet V2 } \\
\hline 300 & 0.9899 & 0.9315 & 1 \\
\hline 600 & 0.9831 & 0.8972 & 1 \\
\hline \multicolumn{4}{|l|}{ Inception V2 } \\
\hline \multicolumn{4}{|l|}{300} \\
\hline 600 & 0.9905 & 0.9749 & 1 \\
\hline \multicolumn{4}{|l|}{ MobileNet } \\
\hline 300 & 1 & 1 & 1 \\
\hline 600 & 1 & 1 & 1 \\
\hline \multicolumn{4}{|l|}{ Resnet 101} \\
\hline 300 & 0.9731 & 0.8850 & 1 \\
\hline 600 & 0.8845 & 0.5912 & 1 \\
\hline
\end{tabular}






Fig. 1. DEA Managerial method maximum, minimum and average efficiency over different detection techniques based on the resolution of the input images.

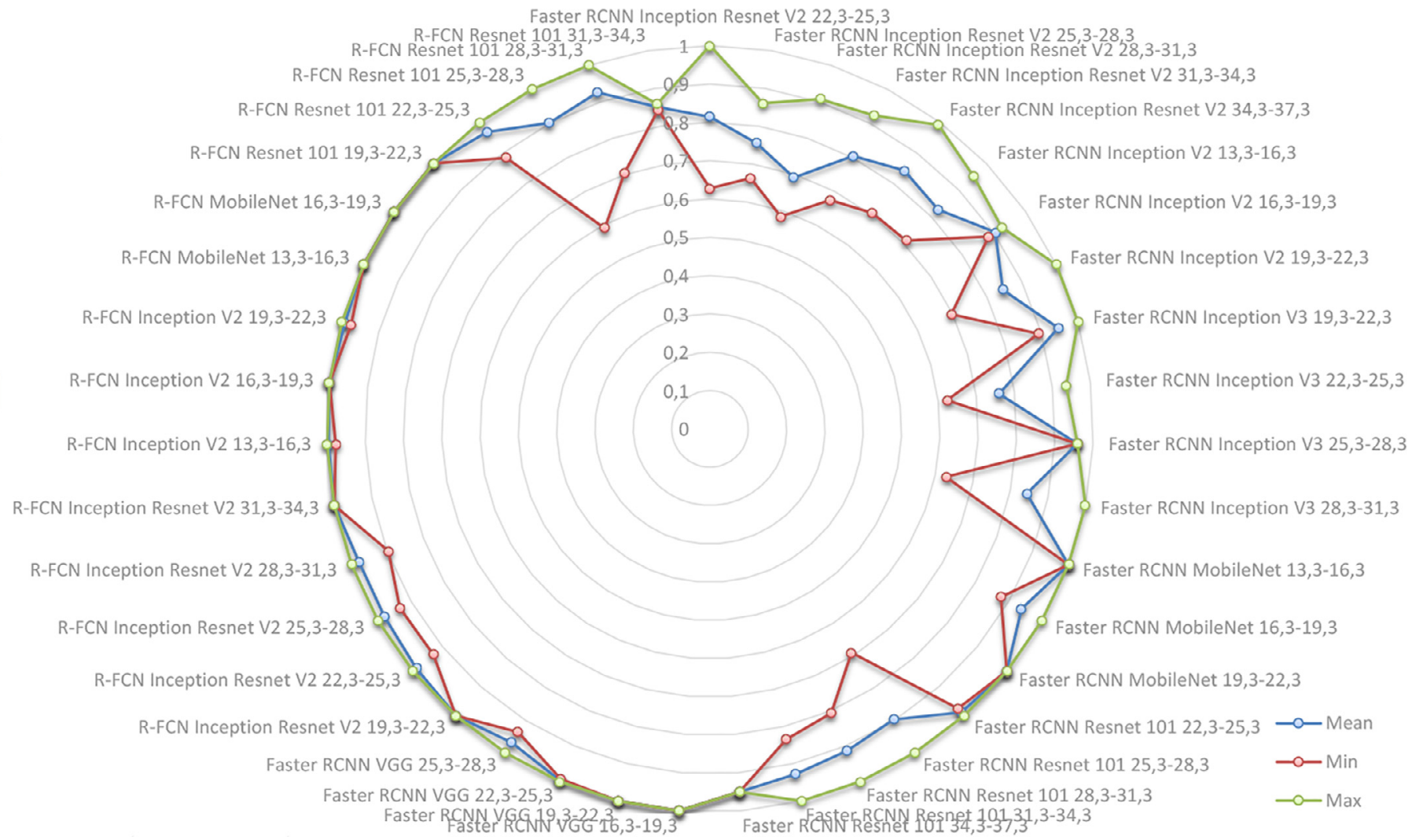

Fig. 2. DEA Managerial method maximum, minimum and average efficiency over different detection techniques based on the box detection (mAP) accuracy. 


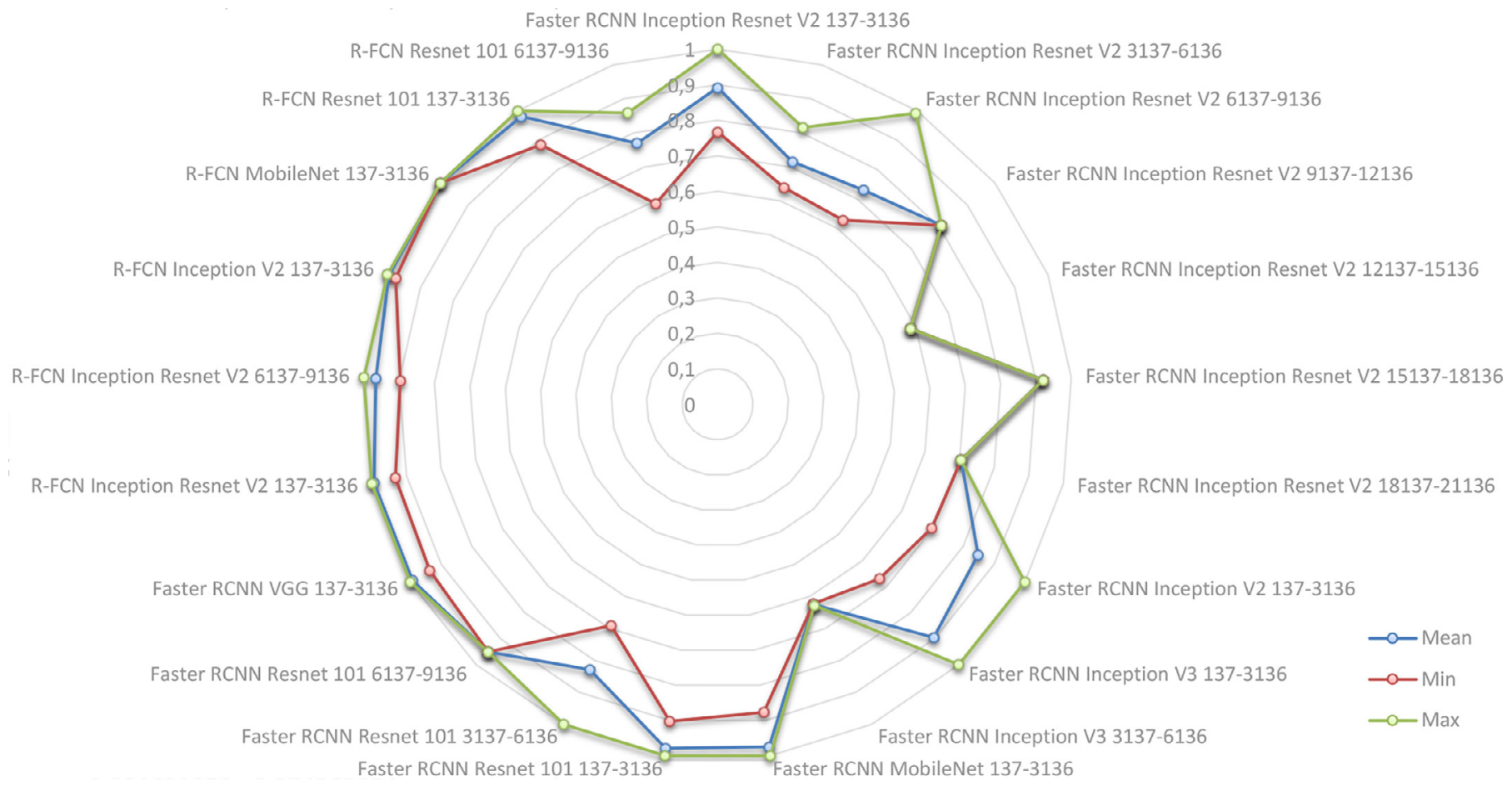

Fig. 3. DEA Managerial method maximum, minimum and average efficiency over different detection techniques based on the consumed memory.

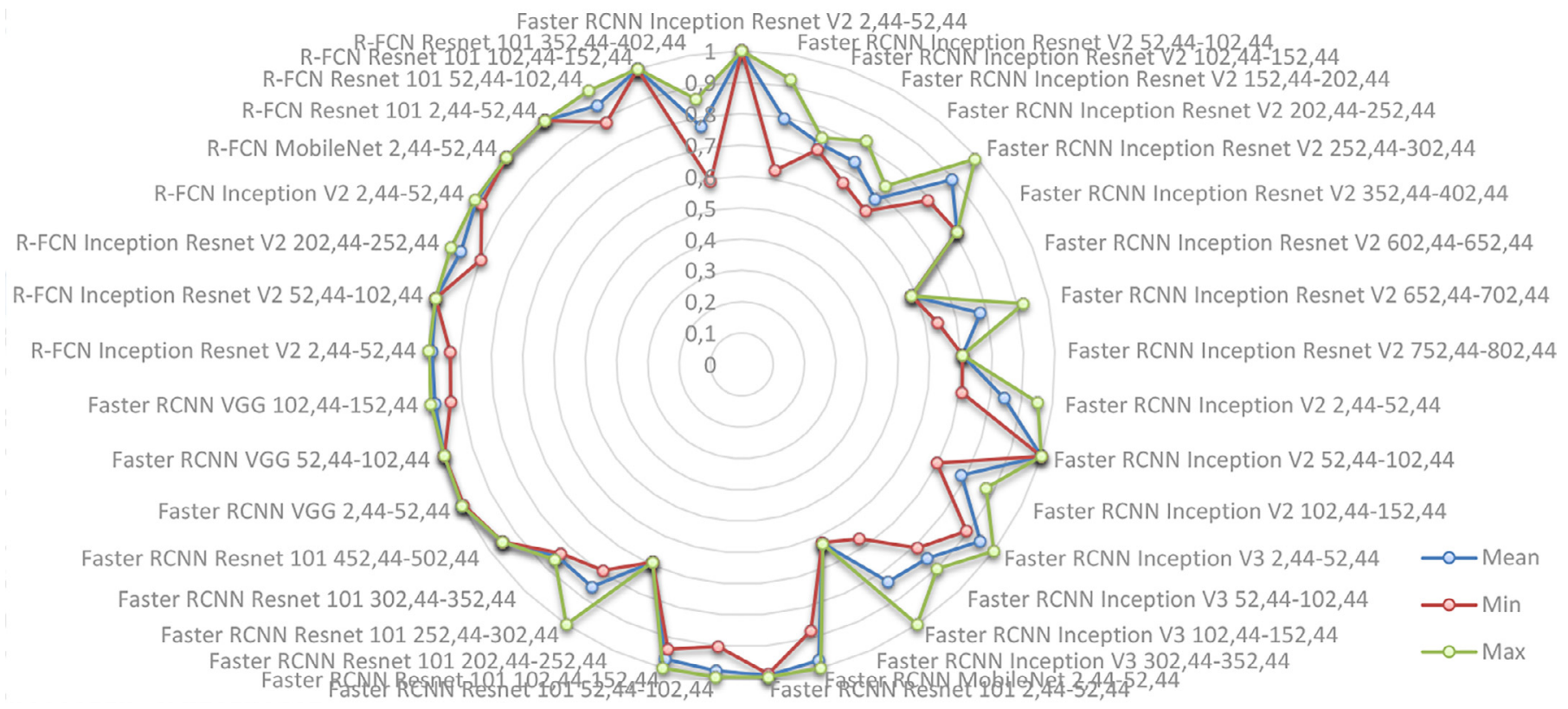

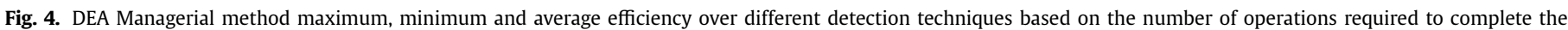
whole process.

parameters shown in Table 1, corresponding to the settings of each architecture analysed.

A representative sample of the data set is shown in Table 2. Note that some parameters of the networks have been omitted from Table 2 to increase clarity. Yet, these parameters are described in the next section in order to subsequently compare the efficiency criteria.

The classification as inputs and outputs of these parameters to perform the DEA analysis is presented in the following section.

\subsection{DEA inputs and outputs}

Once data set has been presented, we discuss how the parameters described in Section 4.1 are taken as inputs or outputs for DEA analysis. Both the number of outputs and inputs may be scaled in order to obtain more information about the efficiency frontier. Nonetheless, due to the temporary cost of the training, these data were reused from previous studies in order to apply the DEA analysis. Moreover, a great advantage of this analysis system is 


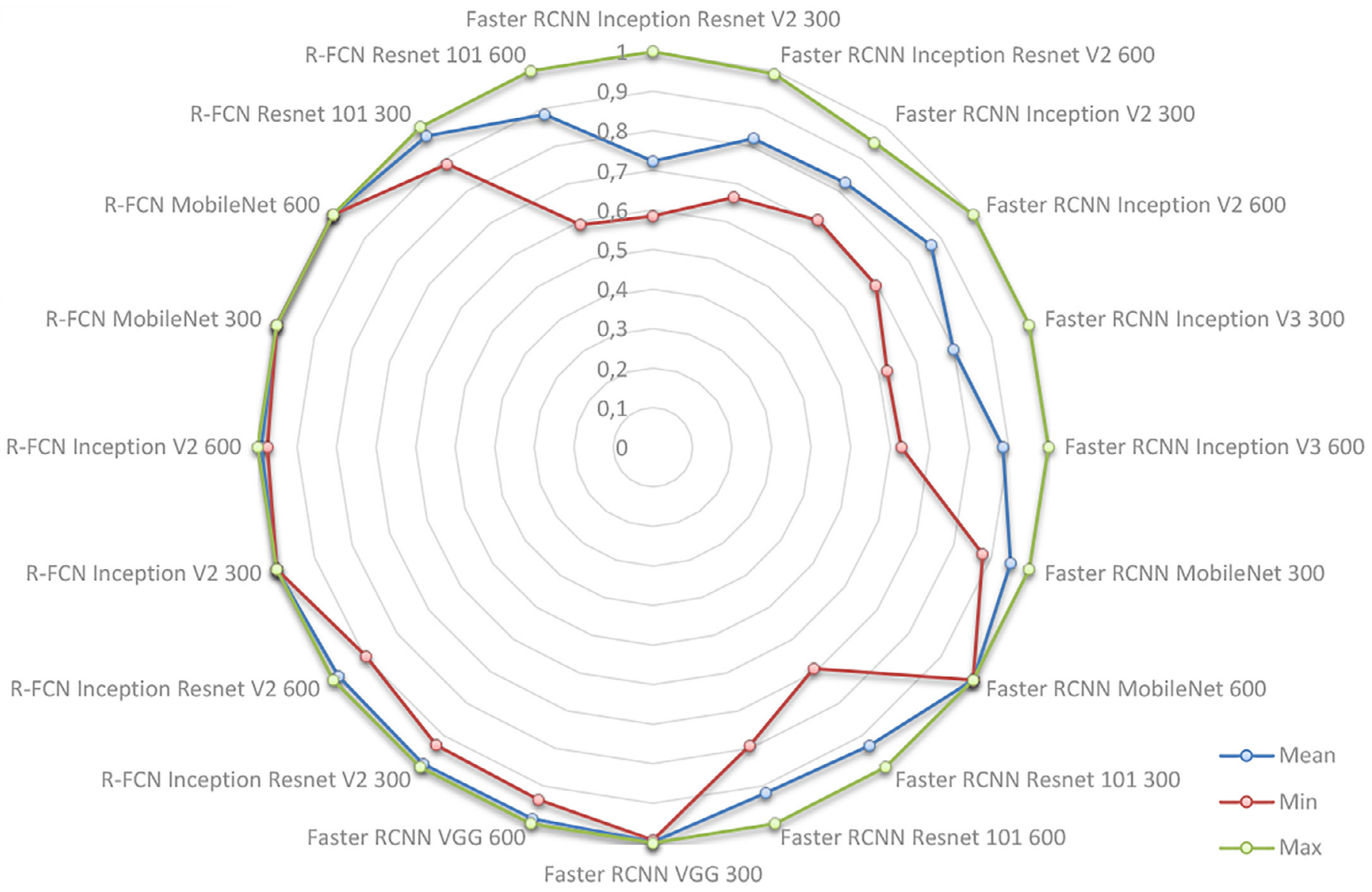

Fig. 5. DEA probabilistic method maximum, minimum and average efficiency over different detection techniques based on the resolution of the input images.

that, due to its relatively low computational cost, it is completely feasible to incorporate new architectures into the system with different configuration values. Therefore, we are able to determine the effect of these new architectures on the efficiency frontier.

The inputs considered in this study are: (a) resolution of the images with which the model works, in pixels; (b) number of regions of interest sent to the classifier for each image (proposals sent to box classifier); and (c) millions of model parameters where (a) and (c) are considered as deterministic inputs. Other parameters such as the number of convolutional layers (RELU, dropout or pooling layers), and the number of features extracted directly from the image have not been analysed.

DEA analysis considers two groups of outputs, as shown in Table 1: positive outputs (good) and negative (bad). The first group refers to the set of outputs that, the higher their value, the better impact they have on the performance of the selected model. On the other hand, the negative outputs represent the set of outputs that, the lower their value, the better impact they have on the performance of the model.

Regarding the positive outputs of the considered models, they are related to the average precision (mAP) with the following different considerations: (i) general $\mathrm{mAP}$ for the whole set of images in the validation set; (ii) $\mathrm{mAP}$ depending on the size of the region of interest detected (large, medium or small); and (iii) mAP @. 75IOU and mAP @. 50IOU, which refer to the average precision with an overlapping threshold between the ground truth and the bounding box generated of $70 \%$ and $50 \%$, respectively.

As for the negative outputs, 7 characteristics of the considered models were taken into account: (i) memory consumption during learning process $(\mathrm{MiB})$; (ii) floating point operations required for training; (iii) CPU time consumed by each batch (milliseconds); (iv) memory consumption during learning process (MiB); (v) average deviation of memory consumption (MiB); (vi) time deviation of CPU consumption (milliseconds); and (vii) time and deviation of GPU utilisation.

Once the data set and the inputs and outputs that make up the input parameters of the DEA analysis have been presented, this analysis has been applied to the previous data. Since this paper addresses 3 different methodologies for the efficiency analysis, the results for each of these methodologies are presented below.

\subsection{Managerial DEA analysis}

The original managerial DEA analysis was the first model applied to the data described above. This analysis resulted in 69 of the 139 models as belonging to the decision boundary. This means that these 69 models with a maximum efficiency (belong to the efficiency frontier), will be taken as a reference for the projection of inputs and outputs from other non-efficient models.

An example of this process can be seen in the case of the Faster RCNN architecture with Inception V2 with an image resolution of 600 pixels and 300 proposals sent to box classifier (efficiency value of $69,7 \%$ ), respecting to R-FCN architecture with Resnet 101 with an image resolution of 300 pixels and 20 proposals sent to box classifier ( $100 \%$ efficiency value). It should be borne in mind that this process is a multivariate analysis and is quite difficult to figure out with the naked eye. If outputs of these both units are explored, the average precision of the first falls to $21.9 \%$, while the 


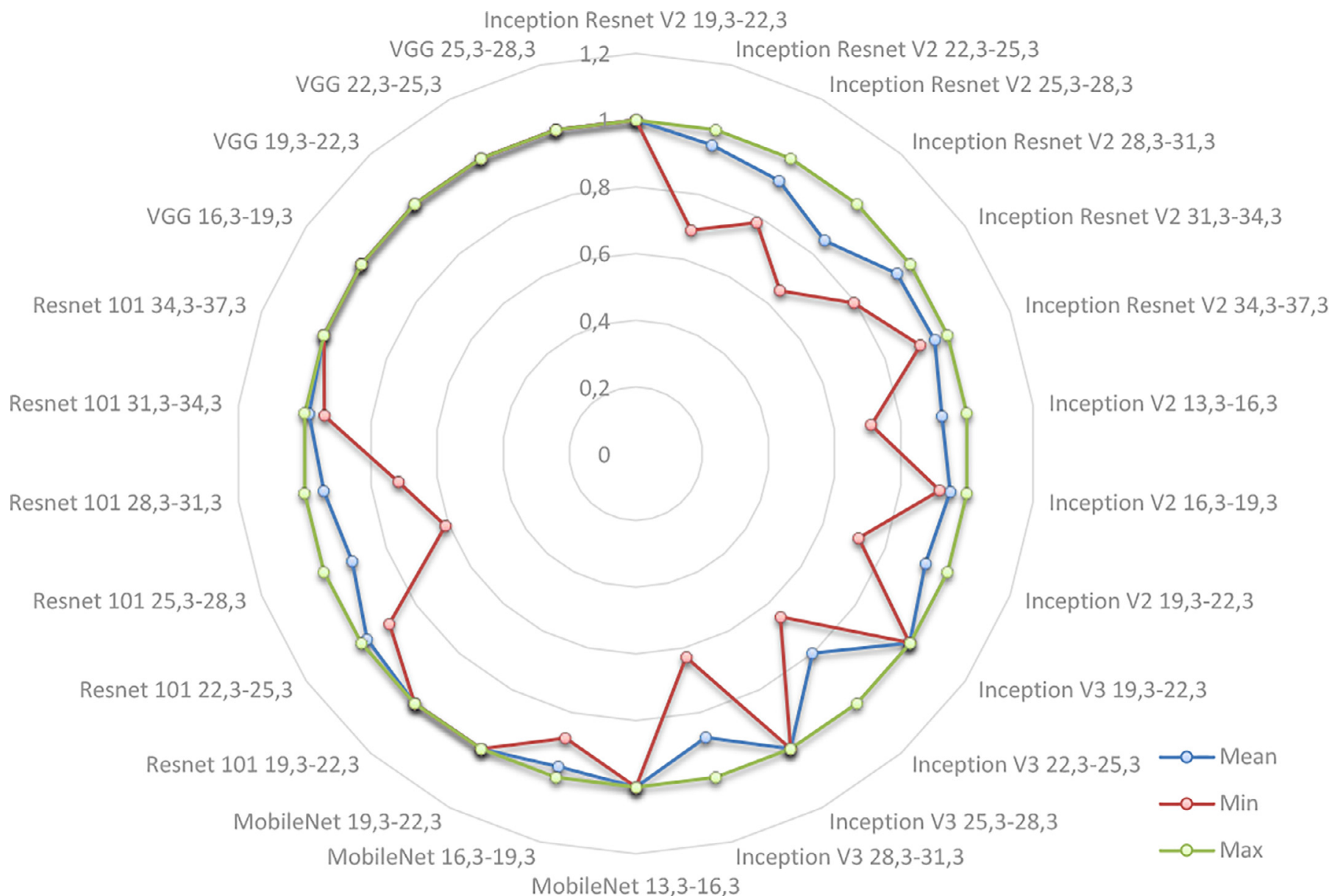

Fig. 6. DEA probabilistic method maximum, minimum and average efficiency over different detection techniques based on the box detection (mAP) accuracy.

second rises to $30.1 \%$. This is also reflected in the average accuracy measured on different image sizes. In contrast, the memory consumption of the Faster RCNN based model is much higher than in the R-FCN. As DEA analysis considers both the outputs and the inputs to produce the efficiency frontier, in this case the increase in detection accuracy counteracts the increase in computational capabilities needed to execute the process of training the model. To better illustrate the comparison of the efficiency values provided by the DEA analysis on the two selected models, an analysis on the accuracy percentiles for both models on the overall result is performed. In R-FCN Resnet 101, the accuracy is over the 90th percentile, while in the case of Faster RCNN with Inception V2, it falls below the 30th percentile. When the same method is employed to calculate the percentiles in terms of memory consumption, both Faster RCNN and R-FCN provide results within the 80th percentile. Although this justification is merely illustrative and it does not reference the complexity of DEA analysis, it shows the utility of this methodology to define efficiency frontiers in a multivariate data scenario of a high degree of complexity.

To conclude the analysis of the results for the managerial DEA model applied on the data obtained from the detection through the configurations of neural networks under study, we will compare the obtained results. The resolution of the original image, the total accuracy of the detection (mAP), the amount of memory used during the training and, finally, the number of needed operations to carry out the complete training process will be taken as reference. Based on the image resolution, the results presented in Table 3, and illustrated in Fig. 1, denote that methods based on R-FCN are much more stable than those based on Faster
RCNN according to this input. This conclusion is obtained from the analysis of the average values of efficiency. Faster RCNN values for this output are relatively low (around 0.8 ) and unstable, unlike those of R-FCN, whose values are close to 1 . These results lead to the conclusion that, regardless of the original image resolution, algorithms based on R-FCN obtain results that make them belong to the efficiency frontier. However, there are some configurations for Faster RCNN where efficiency coefficient is high. For example, Faster RCNN + MobileNet for an image resolution of $600 \times 600$, where the efficiency value is 1 .

Same happens for the efficiency analysis in terms of accuracy, as shown in Fig. 2, and whose numeric values grouped by mAP intervals and network architecture are shown in Table 4. Nonetheless, in this case it can be observed that those methods with greater precision present an efficiency value close to 1, independently of whether it is R-FCN or Faster RCNN. For the next example, values where grouped by accuracy intervals for each architecture-extractor pair. Example of this are Faster RCNN MobileNet [19.3-22.3] and Faster RCNN Resnet 101 [22.3-25.3].

Concluding with the DEA managerial algorithm, Figs. 3 and 4, which show the existing correlation between the efficiency value and the consumed memory and the necessary operations, respectively, are presented. On one hand, In the first figure, the smaller the amount of memory consumed, the higher the efficiency values tend to be. On the other hand, in the second case, a greater number of operations leads to a decrease of these values. We should not forget that this is an analysis of grouped values, so the possibility that there are specific configurations that do not follow this identified patte $\mathrm{rn}$ is present. 
Table 4

Summary of the efficiency coefficients of the DEA analysis (managerial/probabilistic/Bayesian) grouped by $\mathrm{mAP}$ intervals for each of the analysed architectures.

\begin{tabular}{|c|c|c|c|}
\hline $\begin{array}{l}\text { mAP accuracy } \\
\text { interval }\end{array}$ & $\begin{array}{l}\text { Average DEA } \\
\text { coefficient } \\
\text { man/prob/bay }\end{array}$ & $\begin{array}{l}\text { Min DEA } \\
\text { coefficient } \\
\text { man/prob/bay }\end{array}$ & $\begin{array}{l}\text { Max DEA } \\
\text { coefficient } \\
\text { man/prob/bay }\end{array}$ \\
\hline \multicolumn{3}{|l|}{ Faster RCNN } & Inception Resnet V2 \\
\hline [22.3-25.3] & $0.814 / 0.845 / 0.780$ & $0.628 / 0.691 / 0.577$ & $1.000 / 1.000 / 0.983$ \\
\hline [25.3-28.3] & $0.756 / 0.844 / 0.719$ & $0.663 / 0.781 / 0.595$ & $0.861 / 0.932 / 0.910$ \\
\hline [28.3-31.3] & $0.692 / 0.748 / 0.637$ & $0.584 / 0.652 / 0.354$ & $0.907 / 0.947 / 0.934$ \\
\hline [31.3-34.3] & $0.805 / 0.901 / 0.822$ & $0.675 / 0.794 / 0.641$ & $0.923 / 0.972 / 0.965$ \\
\hline [34.3-37.3] & $0.843 / 0.958 / 0.713$ & $0.705 / 0.913 / 0.404$ & $0.993 / 1.000 / 0.978$ \\
\hline \multicolumn{4}{|l|}{ Inception V2 } \\
\hline [13.3-16.3] & $0.826 / 0.836 / 0.741$ & $0.709 / 0.711 / 0.588$ & $0.952 / 0.969 / 0.936$ \\
\hline [16.3-19.3] & $0.904 / 0.924 / 0.904$ & $0.883 / 0.918 / 0.897$ & $0.925 / 0.931 / 0.910$ \\
\hline [19.3-22.3] & $0.846 / 0.857 / 0.821$ & $0.697 / 0.714 / 0.682$ & $1.000 / 1.000 / 0.902$ \\
\hline \multicolumn{4}{|l|}{ Inception V3 } \\
\hline [19.3-22.3] & $0.946 / 0.999 / 0.978$ & $0.892 / 0.997 / 0.960$ & $1.000 / 1.000 / 0.997$ \\
\hline [22.3-25.3] & $0.760 / 0.799 / 0.715$ & $0.623 / 0.654 / 0.450$ & $0.936 / 1.000 / 0.960$ \\
\hline [25.3-28.3] & $0.959 / 1.000 / 0.994$ & $0.959 / 1.000 / 0.994$ & $0.959 / 1.000 / 0.994$ \\
\hline [28.3-31.3] & $0.843 / 0.876 / 0.800$ & $0.628 / 0.629 / 0.528$ & $1.000 / 1.000 / 0.967$ \\
\hline \multicolumn{4}{|l|}{ MobileNet } \\
\hline [13.3-16.3] & $1.000 / 1.000 / 0.993$ & $1.000 / 1.000 / 0.983$ & $1.000 / 1.000 / 1.000$ \\
\hline & $0.938 / 0.942 / 0.955$ & $0.877 / 0.879 / 0.893$ & $1.000 / 1.000 / 1.000$ \\
\hline [19.3-22.3] & $1.000 / 1.000 / 0.995$ & $1.000 / 1.000 / 0.992$ & $1.000 / 1.000 / 1.000$ \\
\hline \multicolumn{4}{|l|}{ Resnet 101} \\
\hline [22.3-25.3] & $0.988 / 0.993 / 0.981$ & $0.975 / 0.980 / 0.945$ & $1.000 / 1.000 / 1.000$ \\
\hline [25.3-28.3] & $0.898 / 0.910 / 0.896$ & $0.691 / 0.722 / 0.713$ & $1.000 / 1.000 / 0.991$ \\
\hline [28.3-31.3] & $0.913 / 0.934 / 0.900$ & $0.806 / 0.837 / 0.801$ & $1.000 / 1.000 / 0.987$ \\
\hline [31.3-34.3] & $0.928 / 0.982 / 0.941$ & $0.834 / 0.940 / 0.887$ & $1.000 / 1.000 / 0.990$ \\
\hline [34.3-37.3] & $0.950 / 1.000 / 0.940$ & $0.950 / 1.000 / 0.940$ & $0.950 / 1.000 / 0.940$ \\
\hline \multicolumn{4}{|l|}{ VGG } \\
\hline [16.3-19.3] & $1.000 / 1.000 / 0.970$ & $1.000 / 1.000 / 0.970$ & $1.000 / 1.000 / 0.970$ \\
\hline [19.3-22.3] & $1.000 / 1.000 / 0.997$ & $1.000 / 1.000 / 0.994$ & $1.000 / 1.000 / 1.000$ \\
\hline [22.3-25.3] & $0.997 / 1.000 / 0.991$ & $0.993 / 1.000 / 0.973$ & $1.000 / 1.000 / 1.000$ \\
\hline [25.3-28.3] & $0.968 / 1.000 / 0.983$ & $0.937 / 1.000 / 0.972$ & $1.000 / 1.000 / 0.994$ \\
\hline \multirow{2}{*}{\multicolumn{4}{|c|}{$\begin{array}{l}\text { R-FCN } \\
\text { Inception Resnet V2 }\end{array}$}} \\
\hline & & & \\
\hline [19.3-22.3] & $1.000 / 1.000 / 0.997$ & $1.000 / 1.000 / 0.997$ & $1.000 / 1.000 / 0.997$ \\
\hline [22.3-25.3] & $0.988 / 0.989 / 0.950$ & $0.931 / 0.937 / 0.806$ & $1.000 / 1.000 / 1.000$ \\
\hline [25.3-28.3] & $0.980 / 0.988 / 0.912$ & $0.934 / 0.941 / 0.728$ & $1.000 / 1.000 / 0.974$ \\
\hline [28.3-31.3] & $0.979 / 0.980 / 0.924$ & $0.897 / 0.898 / 0.643$ & $1.000 / 1.000 / 0.998$ \\
\hline [31.3-34.3] & $1.000 / 1.000 / 0.8666$ & $1.000 / 1.000 / 0.805$ & $1.000 / 1.000 / 0.943$ \\
\hline \multicolumn{4}{|l|}{ Inception V2 } \\
\hline [13.3-16.3] & 0.996/0.999/0.981 & $0.977 / 0.995 / 0.890$ & $1.000 / 1.000 / 1.000$ \\
\hline [16.3-19.3] & $1.000 / 1.000 / 0.957$ & $1.000 / 1.000 / 0.957$ & $1.000 / 1.000 / 0.957$ \\
\hline [19.3-22.3] & $0.992 / 1.000 / 0.979$ & $0.975 / 1.000 / 0.961$ & $1.000 / 1.000 / 0.998$ \\
\hline \multicolumn{4}{|l|}{ MobileNet } \\
\hline [13.3-16.3] & $1.000 / 1.000 / 0.984$ & $1.000 / 1.000 / 0.920$ & $1.000 / 1.000 / 1.000$ \\
\hline [16.3-19.3] & $1.000 / 1.000 / 0.994$ & $1.000 / 1.000 / 0.992$ & $1.000 / 1.000 / 0.996$ \\
\hline \multicolumn{4}{|l|}{ Resnet 101} \\
\hline [19.3-22.3] & $0.929 / 1.000 / 0.995$ & $0.591 / 1.000 / 0.995$ & $1.000 / 1.000 / 0.995$ \\
\hline [22.3-25.3] & $0.929 / 0.972 / 0.945$ & $0.591 / 0.897 / 0.801$ & $1.000 / 1.000 / 1.000$ \\
\hline [25.3-28.3] & $0.929 / 0.908 / 0.853$ & $0.591 / 0.610 / 0.520$ & $1.000 / 1.000 / 0.993$ \\
\hline [28.3-31.3] & $0.929 / 0.948 / 0.893$ & $0.591 / 0.716 / 0.617$ & $1.000 / 1.000 / 1.000$ \\
\hline [31.3-34.3] & $0.929 / 1.000 / 0.810$ & $0.591 / 1.000 / 0.773$ & $1.000 / 1.000 / 0.847$ \\
\hline
\end{tabular}

\subsection{Probabilistic DEA analysis}

Similar results to the previous ones are shown when applying the probabilistic DEA technique to the input data. In this case, the R-FCN instance belongs again to the efficiency frontier (DEA value equal to 1), while Faster RCNN maintains a similar value, although lightly higher, in the result of the analysis methodology. Another difference between the results of the probabilistic DEA and the Managerial applied previously is the number of DMUs that belong to the efficiency frontier. This number of DMUs has increased from 69 to $85(18 \%)$. This is due to the inclusion of a tolerance margin for the probabilistic DEA model, since this model assumes that data follow a probabilistic distribution.
Figs. 5-8 illustrate the probabilistic DEA analysis results. As these figures show, correlation between both algorithms (probabilistic and managerial) exists. However, there is a clear difference between both models. According to the probabilistic DEA model, efficiency values are subtly higher than those obtained with the managerial method. Except this, the previous conclusions and the existing correlation between the inputs and outputs of the model are also maintained in the case of probabilistic DEA.

These results can also be found in Table 4, where, as in the previous section, an analysis of the efficiency values obtained by performing the probabilistic DEA analysis grouped by average accuracy of the architectures.

\subsection{Bayesian DEA analysis}

Finally, the Bayesian DEA model is applied to the same data set. This time, the same two previous DMUs (Faster RCNN architecture with Inception V2 with an image resolution of 600 pixels and 300 proposals sent to box classifier and R-FCN architecture with Resnet 101 with an image resolution of 300 pixels and 20 proposals sent to box classifier) have been compared one more time. This execution shows that both efficiency values have decreased and none of the two units belongs to the efficiency frontier. However, the R-FCN unit shows a very efficient value (0.9994). Comparing the whole set of efficient DMUs, the results present the same trend as in the direct comparison between the two selected DMUs: the number of efficient values decreased from 85 to only 6. As discussed above during the formal description of the Bayesian DEA analysis method, this is the result of an iterative execution of the DEA with a lightly variation in the data set (both inputs and outputs) that follow the probabilistic distribution also described in this section. This iterative execution increases the probability that (1) the results of DEA analysis in a specific DMU could obtain values different than 1 in, at least one iteration; and (2) when modifying the inputs and outputs, the resulting value of the analysis will vary slightly, enough for the exclusion of certain DMUs from the efficiency frontier.

Finally, Figs. 9-12 represent the results of the previous process using the Bayesian DEA model. Unlike the probabilistic model, it can be observed how the number of efficient units has been significantly reduced this time. In any case, the figures show similar patterns to those obtained previously, denoting that architectures based on R-FCN have higher values for efficiency, many of them belonging to the efficiency frontier. In contrast, by reducing the number of DMUs in the efficiency frontier, the comparison capacity between the architectures is improved, enabling the obtention of those configurations that maximise efficiency in a more robust and stable way than with the previous techniques based on probabilistic and managerial DEA.

These results can also be found in Table 4, where the analysis of the efficiency values obtained by the Bayesian DEA according to the average accuracy of the architectures has been performed.

\subsection{Efficiency projections}

DEA not only determines the level of efficiency of the current DMU based on the rest of the DMUs analysed, but also makes it possible to identify which parameters of the proposed architecture should be modified to make the system more efficient: first by modifying input values, and then the outputs if necessary.

Table 5 shows the correction values for DMU \#35, which had an initial efficiency of $61 \%$. In this table can be seen how DEA provides with a set of corrections on the original values to convert this DMU into an efficient unit. The modified variable appears in the "Variable" column. The columns "original value" and "radial movement" 




Fig. 7. DEA probabilistic method maximum, minimum and average efficiency over different detection techniques based on the consumed memory.

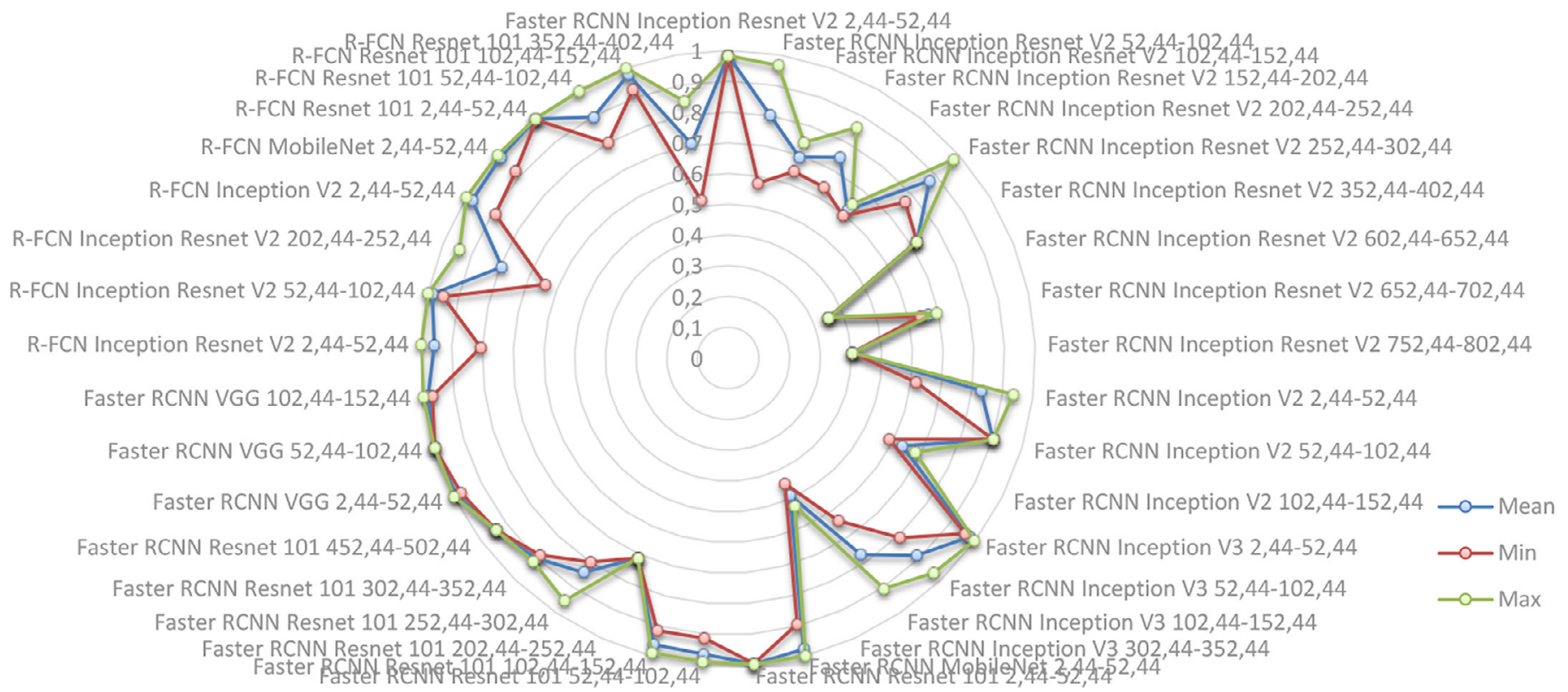

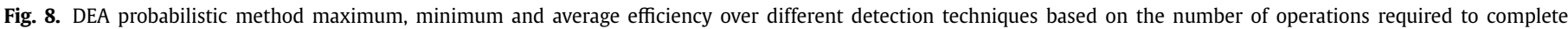
the whole process.

contain the initial value of the variable and the percentage of correction on such value, respectively. The "slack movement" column reflects the absolute value of the resulting correction and, finally, "projected value" column presents the value that this variable should have in that unit to become efficient. Some of these values may not be consistent, as for example a negative value for used memory, as is the case shown in Table 5. This is because the DEA model employed does not offer restrictions on the input and output values, which could result in inconsistent values. This fact must be interpreted at a high level, getting to reduce that value as much as possible whenever possible. If these values are tuned and they approach the optimal value of the projection, the efficiency will increase.

To continue, the correlation of the previously selected DMU with the rest of the DMUs will be compared. By means of this process, the behaviour of inputs and outputs of other DMUs can be "mocked" in order to get the initial DMU to turn efficient, performing a mix between the different behaviours of efficient DMUs. Table 6 shows the results of the equivalence analysis. In this analysis, the DMUs related to a given unit are considered, 
Faster RCNN Inception Resnet V2 300

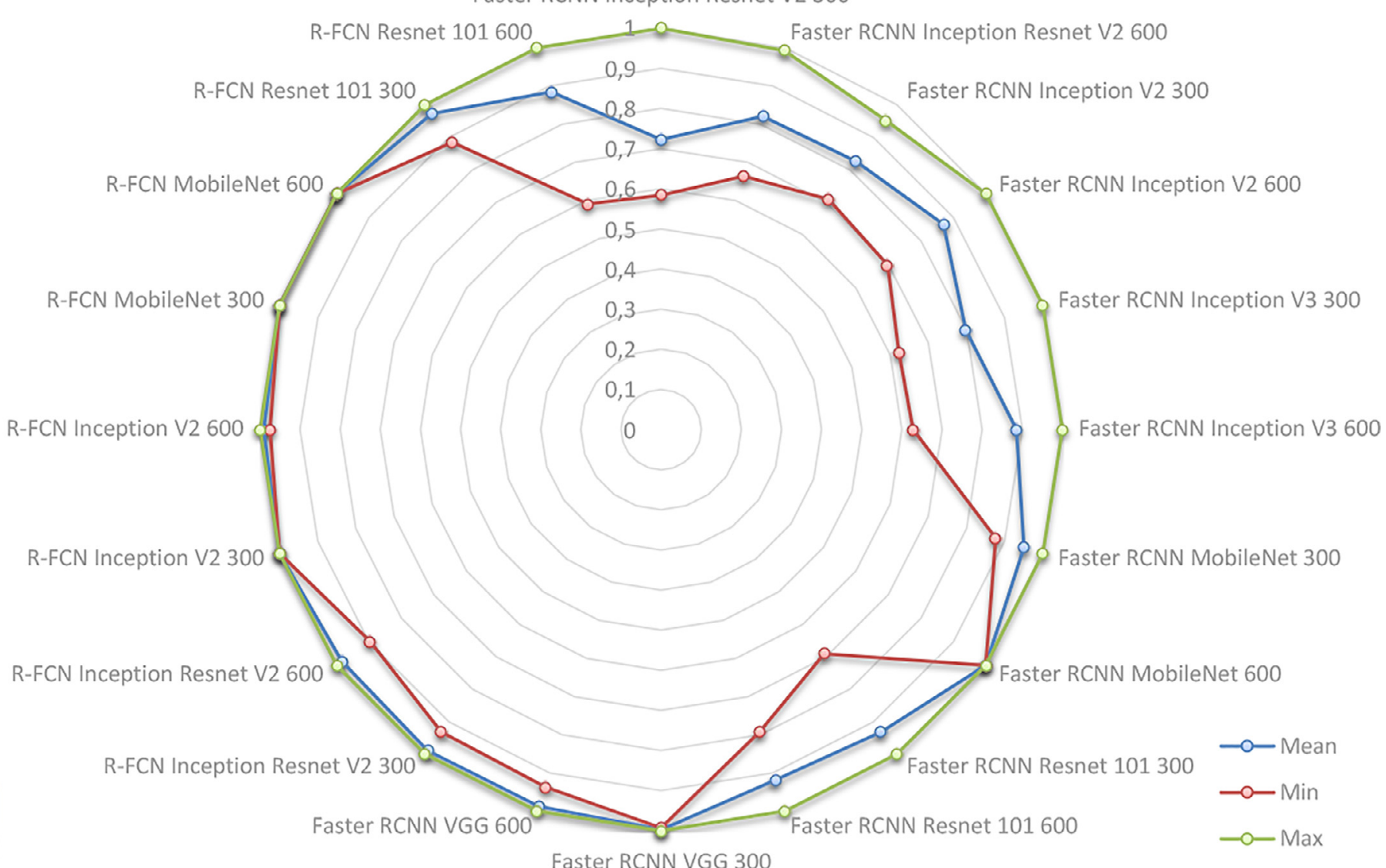

Fig. 9. DEA Bayesian method maximum, minimum and average efficiency over different detection techniques based on the resolution of the input images.

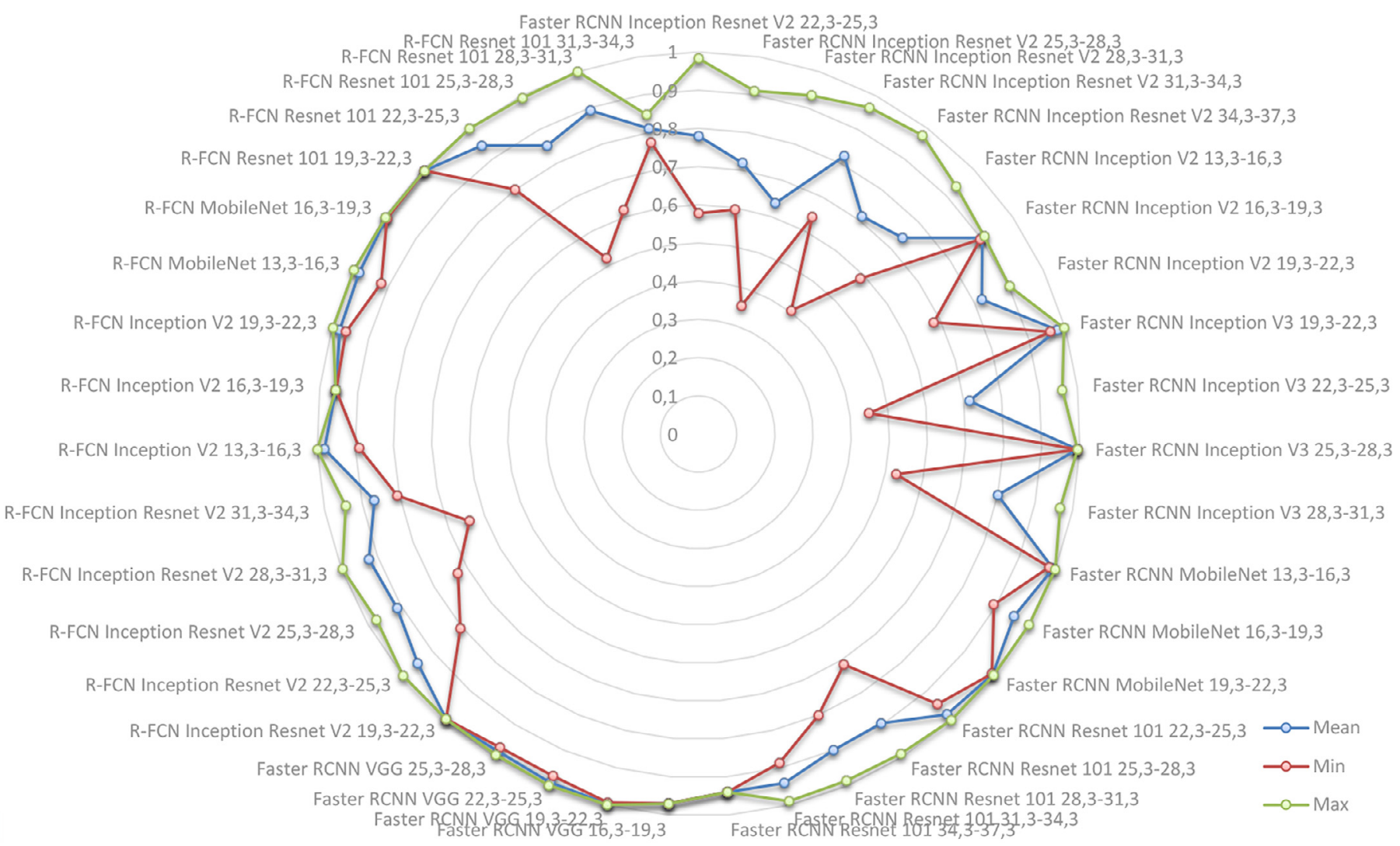

Fig. 10. DEA Bayesian method maximum, minimum and average efficiency over different detection techniques based on the box detection (mAP) accuracy. 


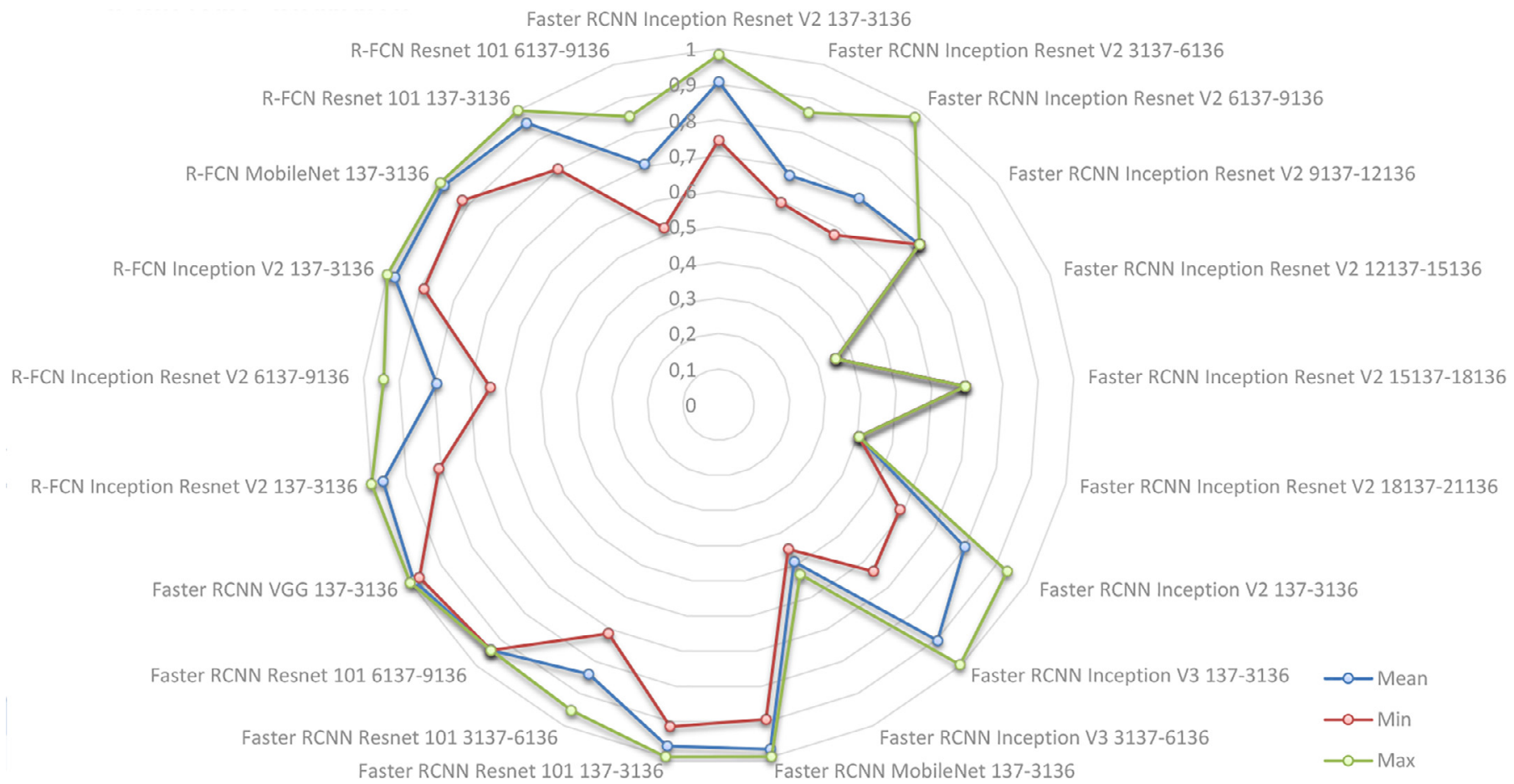

Fig. 11. DEA Bayesian method maximum, minimum and average efficiency over different detection techniques based on the consumed memory.

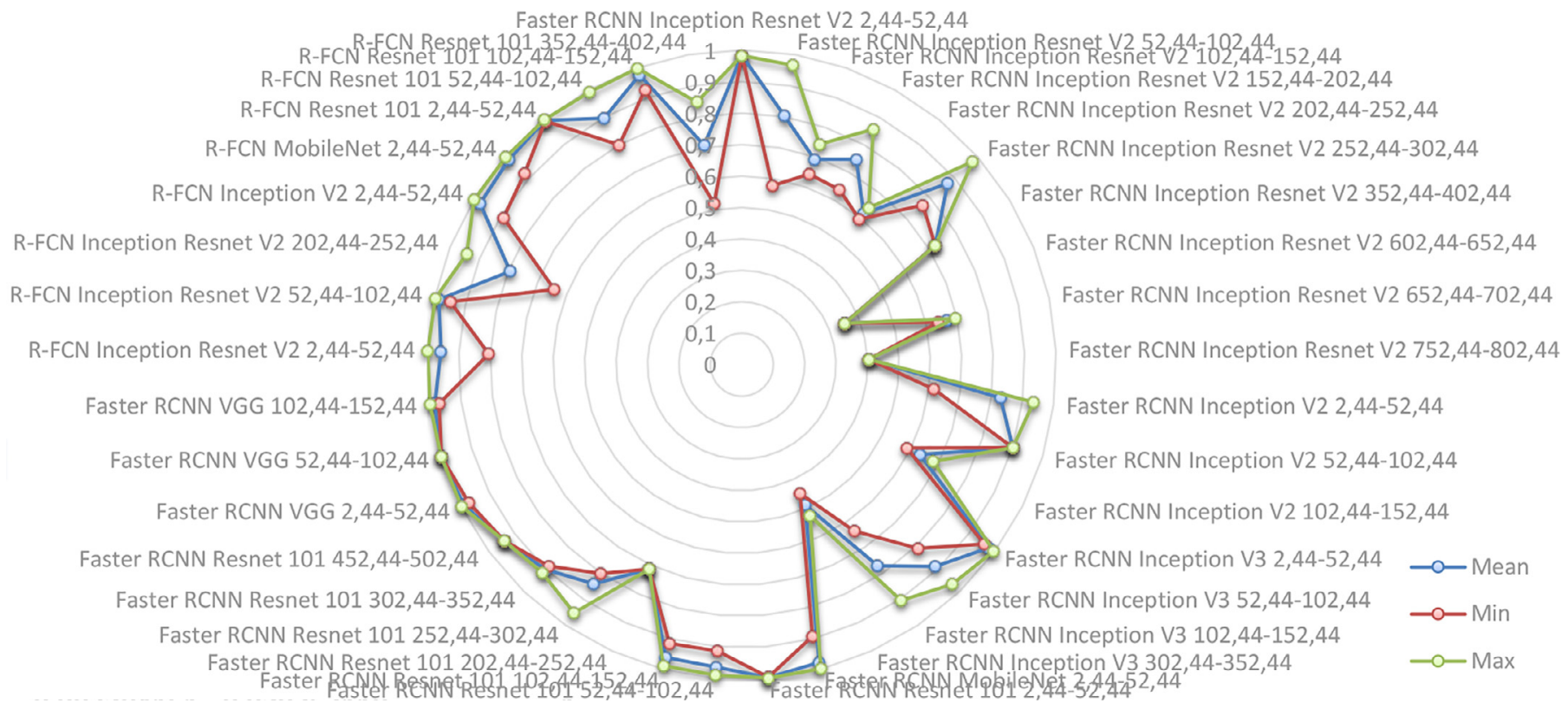

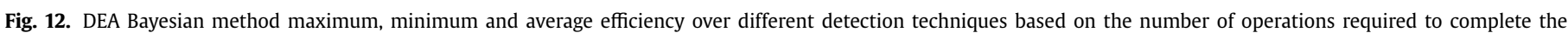
whole process.

so that the percentage of simulation that the selected unit must have to the reference unit is established so that the first one is considered efficient. This allows to establish criteria of change on the data, so that if we want a DMU to become efficient, it is possible to establish how much that DMU should be similar to the rest of the units to achieve that efficiency. The DEA analysis does not directly indicate which are the concrete attributes that must be transformed when establishing that direct equivalence with another DMU, but it is easy to establish this criterion given the optimisation data previously seen.

Finally, the whole set of architectures will be processed in order to identify which inputs and outputs should be optimised (increased in case of positive inputs or outputs and decreased otherwise) in order to transform the DMUs into efficient units through the aforementioned optimisation process. While it is true that the outputs can not be directly changed, this analysis allows 
Table 5

Corrections proposed for DMU \#35 (Faster RCNN inception V2).

\begin{tabular}{|c|c|c|c|c|c|}
\hline \multicolumn{6}{|c|}{$\begin{array}{l}\text { Results for DMU \#35 } \\
\text { Managerial efficiency }=0.6162 \\
\text { Projection summary: }\end{array}$} \\
\hline \multicolumn{2}{|l|}{ Variable } & \multirow{2}{*}{$\begin{array}{l}\begin{array}{l}\text { Original } \\
\text { value }\end{array} \\
300\end{array}$} & \multirow{2}{*}{$\begin{array}{l}\begin{array}{l}\text { Radial } \\
\text { movement }\end{array} \\
-8 \%\end{array}$} & \multirow{2}{*}{$\begin{array}{l}\begin{array}{l}\text { Slack } \\
\text { movement }\end{array} \\
-24.82\end{array}$} & \multirow{2}{*}{$\begin{array}{l}\begin{array}{l}\text { Projected } \\
\text { value }\end{array} \\
275.18\end{array}$} \\
\hline Input & Resolution & & & & \\
\hline Input & \# Proposals & 100 & $-181 \%$ & -181.28 & -81.28 \\
\hline Output good & Overall mAP & 15.3 & $+21 \%$ & +3.19 & 18.49 \\
\hline Output good & mAP large & 27.3 & $+1 \%$ & 0.30 & 27.60 \\
\hline Output good & mAP medium & 7.4 & $+0.5 \%$ & 0.04 & 7.44 \\
\hline Output good & mAP small & 0.5 & $+2.5 \%$ & 0.01 & 0.51 \\
\hline Output good & mAP @. 75IOU & 14.7 & $+0.8 \%$ & 0.12 & 14.82 \\
\hline Output good & mAP @. 50IOU & 18.5 & $+0.8 \%$ & 0.22 & 28.72 \\
\hline Output bad & \# parameters & 13.31 & $-2.9 \%$ & -0.39 & 13.70 \\
\hline Output bad & Memory & 861 & $-100 \%$ & -861.17 & -0.17 \\
\hline Output bad & Memory std & 51 & $-13 \%$ & -6.63 & 44.37 \\
\hline Output bad & $\mathrm{CPU}$ & 535 & $-81.6 \%$ & -436.62 & 98.38 \\
\hline Output bad & CPU std & 7 & $-5.9 \%$ & -0.42 & 7.42 \\
\hline Output bad & GPU & 118 & $-21.8 \%$ & -25.67 & 143.67 \\
\hline Output bad & GPU std & 15 & $-0.5 \%$ & -0.08 & 15.08 \\
\hline Output bad & Flops & 41.95 & $-15.3 \%$ & -6.40 & 35.55 \\
\hline \multicolumn{6}{|c|}{ Listing of peers: } \\
\hline & Peer & \multicolumn{3}{|c|}{ Lambda weight (\%) } & \\
\hline & \#72 & \multicolumn{3}{|c|}{13} & \\
\hline & \#73 & \multicolumn{3}{|c|}{9} & \\
\hline & \#131 & \multicolumn{3}{|c|}{11} & \\
\hline & \#132 & \multicolumn{3}{|c|}{15} & \\
\hline & \#133 & \multicolumn{3}{|c|}{10} & \\
\hline
\end{tabular}

Table 6

Equivalence percentage between 16 selected DMUs (columns) and the rest of the DMUs considered (rows) to transform the selected DMU into an efficient unit.

\begin{tabular}{|c|c|c|c|c|c|c|c|c|c|c|c|c|c|c|c|}
\hline & \#11 (\%) & \#31 (\%) & \#34 (\%) & \#35 (\%) & \#36 (\%) & \#41 (\%) & \#45 (\%) & \#46 (\%) & \#51 (\%) & \#61 (\%) & \#62 (\%) & \#65 (\%) & \#66 (\%) & \#99 (\%) & \#100 (\%) \\
\hline$\# 9$ & 0 & 0 & 0 & 0 & 0 & 0 & 0 & 0 & 0 & 0 & 0 & 0 & 1 & 0 & 0 \\
\hline \#12 & 1 & 0 & 2 & 1 & 0 & 4 & 8 & 1 & 6 & 1 & 17 & 9 & 0 & 0 & 2 \\
\hline$\# 16$ & 0 & 0 & 0 & 0 & 0 & 0 & 0 & 0 & 0 & 0 & 0 & 0 & 0 & 0 & 0 \\
\hline \#19 & 1 & 0 & 0 & 0 & 0 & 0 & 0 & 4 & 0 & 6 & 0 & 2 & 3 & 21 & 15 \\
\hline \#23 & 2 & 0 & 0 & 0 & 0 & 3 & 2 & 1 & 2 & 2 & 1 & 6 & 0 & 1 & 2 \\
\hline \#24 & 2 & 0 & 0 & 0 & 2 & 3 & 1 & 1 & 4 & 2 & 1 & 10 & 1 & 1 & 2 \\
\hline \#26 & 0 & 0 & 0 & 0 & 0 & 0 & 0 & 0 & 0 & 0 & 0 & 0 & 0 & 0 & 0 \\
\hline \#31 & 0 & 13 & 0 & 0 & 0 & 0 & 0 & 0 & 0 & 0 & 0 & 0 & 0 & 0 & 0 \\
\hline \#50 & 0 & 0 & 0 & 0 & 0 & 0 & 0 & 0 & 0 & 0 & 0 & 0 & 0 & 0 & 0 \\
\hline \#56 & 0 & 0 & 0 & 0 & 0 & 0 & 0 & 0 & 0 & 0 & 0 & 0 & 0 & 0 & 0 \\
\hline \#72 & 1 & 4 & 17 & 13 & 0 & 3 & 6 & 0 & 2 & 1 & 5 & 2 & 0 & 0 & 1 \\
\hline \#73 & 1 & 3 & 12 & 9 & 0 & 3 & 5 & 0 & 2 & 0 & 4 & 1 & 0 & 0 & 1 \\
\hline \#78 & 2 & 3 & 3 & 5 & 6 & 4 & 5 & 6 & 9 & 3 & 9 & 11 & 3 & 2 & 6 \\
\hline \#81 & 44 & 6 & 1 & 2 & 31 & 35 & 10 & 30 & 31 & 40 & 11 & 11 & 4 & 0 & 2 \\
\hline \#82 & 8 & 1 & 1 & 1 & 2 & 7 & 14 & 6 & 4 & 10 & 9 & 9 & 1 & 2 & 6 \\
\hline \#83 & 5 & 0 & 1 & 1 & 1 & 5 & 14 & 3 & 2 & 6 & 9 & 7 & 1 & 1 & 6 \\
\hline \#84 & 4 & 1 & 1 & 1 & 0 & 3 & 12 & 1 & 0 & 4 & 4 & 2 & 0 & 1 & 4 \\
\hline \#86 & 4 & 0 & 0 & 0 & 6 & 0 & 0 & 18 & 2 & 10 & 0 & 1 & 38 & 7 & 2 \\
\hline \#87 & 1 & 0 & 0 & 0 & 3 & 0 & 0 & 22 & 11 & 6 & 1 & 12 & 72 & 28 & 15 \\
\hline \#88 & 2 & 0 & 0 & 0 & 1 & 0 & 0 & 5 & 0 & 9 & 0 & 2 & 9 & 43 & 28 \\
\hline \#91 & 4 & 0 & 0 & 0 & 7 & 3 & 0 & 5 & 15 & 5 & 0 & 2 & 8 & 0 & 0 \\
\hline \#101 & 6 & 24 & 3 & 7 & 4 & 12 & 3 & 2 & 6 & 5 & 1 & 1 & 1 & 0 & 0 \\
\hline \#106 & 4 & 4 & 0 & 1 & 11 & 5 & 1 & 8 & 12 & 4 & 1 & 1 & 2 & 0 & 0 \\
\hline \#116 & 5 & 0 & 0 & 0 & 3 & 1 & 0 & 4 & 0 & 7 & 1 & 1 & 4 & 0 & 0 \\
\hline \#117 & 2 & 0 & 0 & 0 & 2 & 1 & 0 & 8 & 9 & 3 & 4 & 10 & 19 & 4 & 3 \\
\hline \#119 & 0 & 0 & 0 & 0 & 0 & 0 & 0 & 2 & 12 & 1 & 4 & 9 & 7 & 2 & 2 \\
\hline \#131 & 8 & 36 & 5 & 11 & 8 & 17 & 4 & 3 & 11 & 5 & 1 & 1 & 1 & 0 & 0 \\
\hline \#132 & 2 & 8 & 13 & 15 & 1 & 6 & 8 & 1 & 4 & 2 & 3 & 2 & 0 & 0 & 1 \\
\hline \#133 & 0 & 3 & 11 & 10 & 0 & 3 & 4 & 0 & 2 & 1 & 2 & 1 & 0 & 0 & 0 \\
\hline
\end{tabular}

the establishment of an objective criterion which improves the efficiency of the considered architectures.

\section{Conclusions and future work}

In this paper we propose a low-computing-demanding model based on DEA for the measurement of the efficiency (performance) of different object-detection architectures, and to provide a set of tools for the improvement of different configurations of neural networks based on the most relevant input and output parameters. Specifically, 139 configurations of neural networks used for object detection in images have been processed based on 16 parameters: 3 inputs and 13 outputs. Three different techniques for the computation of efficiency have been presented for this analysis in order to increase the robustness: Managerial DEA, Probabilistic DEA and Bayesian DEA. The three techniques have been applied on 


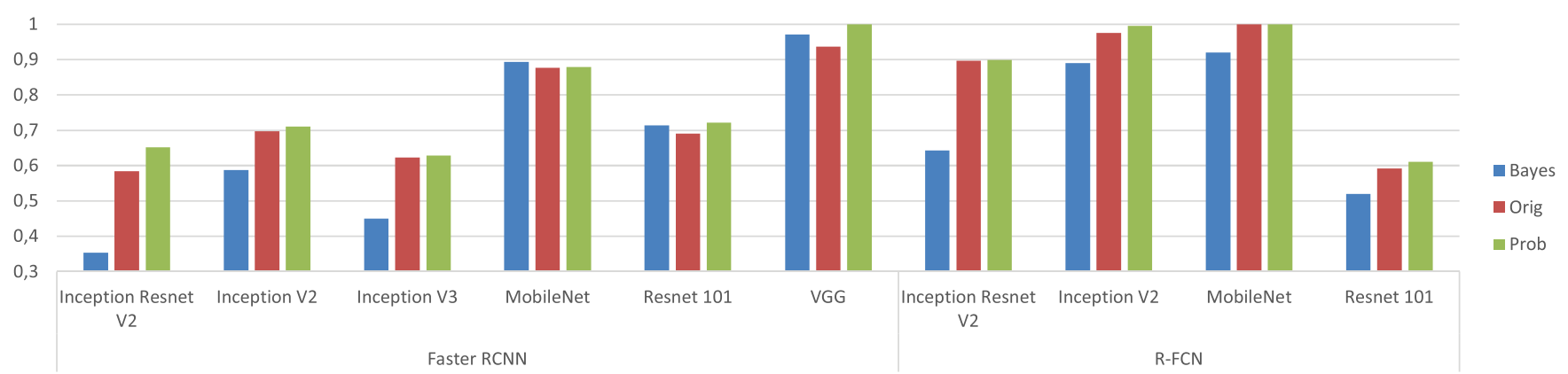

Fig. 13. Average efficiency values for each of the detection techniques grouped by the type of applied DEA algorithm (Managerial, probabilistic or Bayesian).

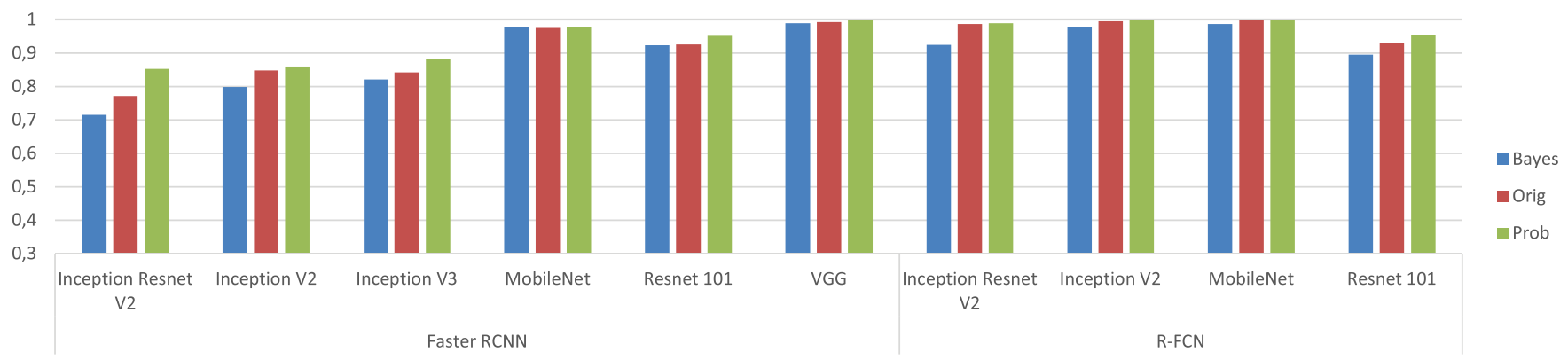

Fig. 14. Minimum efficiency values for each of the detection techniques grouped by the type of applied DEA algorithm (Managerial, probabilistic or Bayesian).

several neural-network configurations based on R-FCN Inception V2, R-FCN MobileNet, R-FCN Resnet and R-FCN Inception Resnet which places them at the optimization frontier for inputs and outputs values presented. It is also demonstrated after the application of these efficiency calculation and projection techniques that, as could be expected, R-FCN Inception Resnet generally obtains the highest values in terms of efficiency. Regarding the different DEA techniques introduced in this paper, it should be noticed that the probabilistic DEA, in general, tends to maximise the number of DMUs present in the efficiency frontier, unlike the Bayesian DEA, which reduces the number of units in such frontier. The latter allows, therefore, the selection of those DMUs that belong to the efficiency set in a more restrictive way, and, in consequence, to point out the optimum efficiency of the rest of the units. This can be seen in Figs. 13 and 14. As mentioned in Section 3, one of the original DEA limitations is that since it is an exclusively mathematical technique, random noise effects over data source are not considered in this DEA approach. In the probabilistic and Bayesian DEA, a statistical approach is adopted and therefore these factors are taken into account, when considering the observed data as collection of random variables. As we can see, the results obtained when applying the three techniques are similar, which is an indication of robustness, that is, the results are not sensitive to random variation effects. On the other hand, in the results obtained after the application of the algorithms, we verify that the standard errors of the Bayesian estimated efficiencies, in general, are small enough compared to the estimated values (that is, the estimations are very accurate), which means also that the results obtained are robust against possible random effects. Finally, this novel comparison methodology for units based on neural-network architectures, allows to determine whether a given architecture belongs to the most-efficient architectures set or may be improved based on the values of its inputs and outputs. In this way, this methodology also enables the determination of the input values to be improved or the values of the outputs that must be reached in order to make efficient a non-efficient unit. It should be noted that the calculation of the efficiency frontier using the presented DEA techniques is computationally undemanding, so it is possible to feed back the data set and launch the process of computing the frontier without major inconveniences. In contrast, the computational and temporal cost of training a neural network based on the trial-error method is extremely high, hence the usefulness of this exposed methodology. New meta-architectures [47-49] may employ this method to determine their relative efficiency and check which input can be improved to achieve the frontier results. In this way, a specific architecture could be chosen based on the characteristics of the proposed inputs, giving a fairly clear approximation of how the selected architecture will behave based on the features of the given inputs.

As future work, this paper sets a milestone for a new research track which may be expanded in the following three main directions:

- Application of DEA models to more recent object detection architectures so that the results and conclusions of this work can be confirmed or contrasted;

- Development of models which may employ DEA models to improve the efficiency for the training of neural networks; and

- Development of expert systems equipped with low-computingdemanding DEA models for the dynamic application of object detection architectures depending on the scenario.

\section{Intellectual property}

We confirm that we have given due consideration to the protection of intellectual property associated with this work and that there are no impediments to publication, including the timing of publication, with respect to intellectual property. In so doing we confirm that we have followed the regulations of our institutions concerning intellectual property.

\section{Research ethics}

We further confirm that any aspect of the work covered in this manuscript that has involved human patients has been conducted with the ethical approval of all relevant bodies and that such approvals are acknowledged within the manuscript. 


\section{Authorship}

We confirm that the order of authors listed in the manuscript has been approved by all named authors. We confirm that the order of authors listed in the manuscript has been approved by all named authors.

\section{Declaration of Competing Interest}

None.

\section{Acknowledgment}

This research is funded by the Spanish Ministry of Economy and Competitiveness through the projects Vision and Crowdsensing Technology for an Optimal Response in Physical-Security (TIN2017-82113-C2-1-R)

\section{Appendix A. Data envelopment analysis formal description}

\section{A.1. Natural and managerial disposability}

In model (A.1), each $j$ th DMU $j=1, \ldots, n$, uses inputs $X_{j}=$ $\left(x_{1 j}, \ldots, x_{m j}\right)^{T}$ to produce desirable outputs $G_{j}=\left(g_{1 j}, \ldots, g_{s j}\right)^{T}$ and undesirable outputs $B_{j}=\left(b_{1 j}, \ldots, b_{h j}\right)^{T}$. Furthermore, $d_{i}^{x}, i=1, \ldots, m, d_{r}^{g}, r=1, \ldots, s$ and $d_{f}^{b}, f=1, \ldots, h$ are all slack variables related to inputs, desirable outputs and undesirable outputs, respectively. $\lambda=\left(\lambda_{1}, \ldots, \lambda_{n}\right)^{T}$ are structural or intensity variables, which are unknown and are used for connecting the input and output vectors by a convex combination. $R$ is the range resolute through the upper and lower bounds of inputs, desirable outputs and undesirable outputs, expressed by:

$R_{i}^{X}=(m+s+h)^{-1}\left(\max \left\{x_{i j} / j=1, \ldots, n\right\}-\min \left\{x_{i j} / j=1, \ldots, n\right\}\right)$

$R_{r}^{g}=(m+s+h)^{-1}\left(\max \left\{g_{r j} / j=1, \ldots, n\right\}-\min \left\{g_{r j} / j=1, \ldots, n\right\}\right)$

and

$R_{f}^{b}=(m+s+h)^{-1}\left(\max \left\{b_{f j} / j=1, \ldots, n\right\}-\min \left\{b_{f j} / j=1, \ldots, n\right\}\right)$

The natural efficiency of the $k$ th policy is evaluated by the following CRS and VRS radial model (see [50] for a better understanding):

$$
\begin{aligned}
& \max \xi+\epsilon\left(\sum_{i=1}^{m} R_{i}^{x} d_{i}^{x}+\sum_{r=1}^{s} R_{r}^{g} d_{i}^{g}+\sum_{f=1}^{h} R_{f}^{b} d_{i}^{b}\right) \\
& \text { s.t. } \quad \sum_{j=1}^{n} x_{i j} \lambda_{j}+d_{i}^{x}=x_{i k}, i=1, \ldots, m, \\
& \sum_{j=1}^{n} g_{r j} \lambda_{j}-d_{r}^{g}-\xi g_{r k}=g_{r k}, r=1, \ldots, s, \\
& \sum_{j=1}^{n} b_{f j} \lambda_{j}+d_{f}^{b}+\xi b_{f k}=b_{f k}, f=1, \ldots, h, \\
& d_{i}^{x} \geq 0, i=1, \ldots, m, \\
& d_{r}^{g} \geq 0, r=1, \ldots, s, \\
& d_{f}^{b} \geq 0, f=1, \ldots, h, \\
& \xi \text { Unrestricted }
\end{aligned}
$$

where: $\xi$, which is an unrestricted parameter, represents an unknown inefficiency score indicating a distance between the efficiency frontier and a particular observed vector of desirable and undesirable outputs; In our case, $\epsilon$ takes a value of 0.0001 to reduce the influence of slack variables for computation reasons. If the restriction $\sum_{j=1}^{n} \lambda_{j}=1$ is added to the model (A.1), then the VRS model is obtained (Model A.1*).

The first restriction in systems (A.1) and (A.1*) seeks values of $\lambda_{j}$ to construct a composite unit, with inputs such that $\sum_{j=1}^{n} x_{i j} \lambda_{j}=-d_{i}^{x}+x_{i k}, i=1, \ldots, m$. The positive slack variables $d_{i}^{x}$ indicate that further decreases in inputs may be performed, which necessarily alter the proportions used, and hence they show the existence of inefficiencies.

Similarly, the second restriction, $\sum_{j=1}^{n} g_{r j} \lambda_{j}=d_{r}^{g}+\xi g_{r k}+$ $g_{r k}, r=1, \ldots, s$, tells us that the desirable outputs may be increased or at least maintained by performing a radial expansion $\xi g_{r k}$ and an increase in the slack variable $d_{r}^{g}$.

Analogously, the third restriction, $\sum_{j=1}^{n} b_{f j} \lambda_{j}=-d_{f}^{b}-\xi b_{f k}+$ $b_{f k}, f=1, \ldots, h$, indicates that we have decreased the inputs, and then we reduce the undesirable outputs both radially and in their slack variables.

The objective function implies that two sources of inefficiency may be identified. Ak-policy is efficient if and only if the following two conditions are satisfied: (a) $\xi=0$; (b) $d_{i}^{x}=0, d_{r}^{g}=0, d_{f}^{b}=0$. In this efficient case, $k$-policy belongs to the efficiency frontier. It fulfils restrictions in equation systems (A.1) and (A.1*), and therefore the objective function is 0 . Otherwise, if it is not efficient, the value of the objective function is greater than 0 , due to possible radial movement and possible displacements in the slack variables.

The natural efficiency is then measured as follows:

$\theta^{*}=1-\left[\xi^{*}+\epsilon\left(\sum_{i=1}^{m} R_{i}^{X} d_{i}^{x^{*}}+\sum_{r=1}^{s} R_{r}^{g} d_{i}^{g^{*}}+\sum_{f=1}^{h} R_{f}^{b} d_{i}^{b^{*}}\right)\right]$

This metric of unified efficiency score takes values between 0 and 1 . If the $k$-policy is efficient, then the objective function of equation systems (A.1) and (A.1*) is 0 , and hence the efficiency score equals $\theta^{*}=1$. All slack variables obtained in the optimality of models (A.1) and (A.1*) indicate the level of inefficiency [50].

The Managerial efficiency of the $k$ th policy is evaluated by the following CRS and VRS radial models [50]:

$$
\begin{aligned}
\text { s.t. } \quad \sum_{j=1}^{n} x_{i j} \lambda_{j}-d_{i}^{x} & =x_{i k}, i=1, \ldots, m, \\
\sum_{j=1}^{n} g_{r j} \lambda_{j}-d_{r}^{g}-\xi g_{r k} & =g_{r k}, r=1, \ldots, s, \\
\sum_{j=1}^{n} b_{f j} \lambda_{j}+d_{f}^{b}+\xi b_{f k} & =b_{f k}, f=1, \ldots, h, \\
d_{i}^{x} & \geq 0, i=1, \ldots, m, \\
d_{r}^{g} & \geq 0, r=1, \ldots, s, \\
d_{f}^{b} & \geq 0, f=1, \ldots, h, \\
\xi & \text { Unrestricted }
\end{aligned}
$$

Similarly, if the restriction $\sum_{j=1}^{n} \lambda_{j}=1$ is added to $\operatorname{Model}$ (A.2), then we obtain a VRS Model (A.2*). VRS models can provide with the returns to scale (RTS) and damage to scale (DTS) metrics (see [50] for a better understanding). It is clear that for the natural efficiency the returns to scale has to be increased and for managerial efficiency the damages to scale has to be decreased. Otherwise the technical units are not working well and should correct the imbalances, using the information of the efficient units to which they have to be similar (peers).

\section{A.2. Chance-constrained DEA.}

In the formulation of chance-constrained DEA we consider the more general case in which both inputs and outputs are random 
variables. Then the problem associated to Natural DEA becomes:

$\max \xi$

s.t.

$$
\begin{gathered}
P\left(\sum_{j=1}^{n} x_{i j} \lambda_{j} \leq x_{i k}\right) \geq 1-\alpha, \quad i=1, \ldots, m \\
P\left(\sum_{j=1}^{n} g_{r j} \lambda_{j} \geq(1+\xi) g_{r k}\right) \geq 1-\alpha, \quad r=1, \ldots, s \\
P\left(\sum_{j=1}^{n} b_{f j} \lambda_{j} \leq(1-\xi) b_{f k}\right) \geq 1-\alpha, \quad f=1, \ldots, h
\end{gathered}
$$

\section{$\xi$ Unrestricted}

where $1-\alpha$ denotes the minimum probability with which the constraints are satisfied. The most common choice is $\alpha=0.05$ (other common values are $\alpha=0.01$ or $\alpha=0.1$ ). It is also possible to take different $\alpha$ values for each constraint.

Some inputs may be deterministic in some particular environments. In such a case, we just have to remove the probability term in the corresponding restrictions. In fact, many applications of chance-constrained DEA consider that the inputs are deterministic and only the outputs are stochastic.

We assume the usual hypothesis that both inputs and outputs follows a multivariate normal distribution, and we use the notation $\mu_{i j}^{x}$ and $\sigma_{i j}^{x}$ for the expected value and the standard deviation of $x_{i j}$, that is, $x_{i j} \sim N\left(\mu_{i j}^{x},\left(\sigma_{i j}^{x}\right)^{2}\right)$. Then, the restrictions of problem (A.3) are converted to certainty equivalents $[45,46,51]$, as follows.

Letśs consider the input restrictions $P\left(\sum_{j=1}^{n} x_{i j} \lambda_{j} \leq x_{i k}\right) \geq$ $1-\alpha, \quad i=1, \ldots, m$. Using the notation $s_{i k}=\sum_{j=1}^{n} x_{i j} \lambda_{j}-x_{i k}$ and $\phi(\cdot)$ for the cumulative distribution function of the standard normal distribution, the $i$ th restriction may be denoted as:

$$
\begin{aligned}
P\left(s_{i k} \leq 0\right) & \geq 1-\alpha \Leftrightarrow P\left(\frac{s_{i k}-E\left[s_{i k}\right]}{\sqrt{\operatorname{var}\left[s_{i k}\right]}} \leq \frac{-E\left[s_{i k}\right]}{\sqrt{\operatorname{var}\left[s_{i k}\right]}}\right) \geq 1-\alpha \\
& \Leftrightarrow \phi\left(\frac{-E\left[s_{i k}\right]}{\sqrt{\operatorname{var}\left[s_{i k}\right]}}\right) \geq 1-\alpha \Leftrightarrow \frac{-E\left[s_{i k}\right]}{\sqrt{\operatorname{var}\left[s_{i k}\right]}} \geq \phi^{-1}(1-\alpha) \\
& \Leftrightarrow E\left[s_{i k}\right]+\phi^{-1}(1-\alpha) \sqrt{\operatorname{var}\left[s_{i k}\right]} \leq 0
\end{aligned}
$$

Because of $E\left[s_{i k}\right]=\sum_{j=1}^{n} E\left[x_{i j}\right] \lambda_{j}-E\left[x_{i k}\right]$, the input restrictions become:

$\sum_{j=1}^{n} E\left[x_{i j}\right] \lambda_{j}+\phi^{-1}(1-\alpha) \sqrt{\operatorname{var}\left[s_{i k}\right]} \leq E\left[x_{i k}\right], \quad i=1, \ldots, m$.

Under the general multivariate normal assumption, a non-linear deterministic equivalent is obtained due to the term $\sqrt{\operatorname{var}\left[s_{i k}\right]}$. The computing time necessary to solve the problem increases considerably. Moreover, simulation techniques are needed for the application of the Bayesian methodology and the problem has to be solved thousands of times. Therefore, the computing time is crucial in practice. We assume the single factor disturbance model of [51] to face this challenge. More explicitly:

$x_{i j}=\mu_{i j}^{x}+\sigma_{i j}^{x} \eta^{x}, i=1, \ldots, m, \quad j=1, \ldots, n$ where $\eta^{x}$ is the only factor related to the inputs, and follows a standard normal distribution. From this hypothesis it is clear that the mean and standard deviation of $x_{i j}$ are $\mu_{i j}^{x}$ and $\sigma_{i j}^{x}$, respectively. Under the single factor disturbance hypothesis, the deterministic equivalent is expressed in terms on absolute value. Specifically:

$$
\begin{aligned}
\operatorname{var}\left[s_{i k}\right] & =\operatorname{var}\left[\left(-\sigma_{i k}^{x}+\sum_{j=1}^{n} \sigma_{i j}^{x} \lambda_{j}\right) \eta^{x}\right]=\left(-\sigma_{i k}^{x}+\sum_{j=1}^{n} \sigma_{i j}^{x} \lambda_{j}\right)^{2} \\
& \Rightarrow \operatorname{var} \sqrt{\left[s_{i k}\right]}=\left|-\sigma_{i k}^{x}+\sum_{j=1}^{n} \sigma_{i j}^{x} \lambda_{j}\right|
\end{aligned}
$$

Therefore, the input constraints may be expressed as:

$\sum_{j=1}^{n} \mu_{i j}^{x} \lambda_{j}+\phi^{-1}(1-\alpha)\left|-\sigma_{i k}^{x}+\sum_{j=1}^{n} \sigma_{i j}^{x} \lambda_{j}\right| \leq \mu_{i k}^{x}, \quad i=1, \ldots, m$

This problem can be transformed into an ordinal linear programming problem [51,52]. First, we may consider:

$$
\begin{aligned}
& q_{1 i}^{X}=\left\{\begin{array}{cc}
-\sigma_{i k}^{X}+\sum_{j=1}^{n} \sigma_{i j}^{X} \lambda_{j} & \text { if }-\sigma_{i k}^{X}+\sum_{j=1}^{n} \sigma_{i j}^{x} \lambda_{j} \geq 0 \\
0 & \text { if }-\sigma_{i k}^{X}+\sum_{j=1}^{n} \sigma_{i j}^{x} \lambda_{j}<0
\end{array}, i=1, \ldots, m\right.
\end{aligned}
$$

Hence, $\left|-\sigma_{i k}^{x}+\sum_{j=1}^{n} \sigma_{i j}^{x} \lambda_{i}\right|$ can be expressed by $q_{1 i}^{x}+q_{2 i}^{x}$, where $q_{1 i}^{x}$ and $q_{2 i}^{x}$ satisfy $q_{1 i}^{x}-q_{2 i}^{x}=-\sigma_{i k}^{x}+\sum_{j=1}^{n} \sigma_{i j}^{x} \lambda_{i}$ and $q_{1 i}^{x} q_{2 i}^{x}=0$; moreover, $q_{1 i}^{X} \geq 0, q_{2 i}^{X} \geq 0$. Thus, the input constraints have to be replaced by:

$$
\left.\begin{array}{l}
\sum_{j=1}^{n} \mu_{i j}^{x} \lambda_{j}+\phi^{-1}(1-\alpha)\left(q_{1 i}^{x}+q_{2 i}^{x}\right) \leq \mu_{i k}^{x} \\
-\sigma_{i k}^{x}+\sum_{j=1}^{n} \sigma_{i j}^{x} \lambda_{j}=q_{1 i}^{x}-q_{2 i}^{x} \\
q_{1 i}^{x} \geq 0, \quad q_{2 i}^{x} \geq 0
\end{array}\right\} i=1, \ldots, m
$$

and then we may include $-\varepsilon\left(q_{1 i}^{x}+q_{2 i}^{x}\right)$ to the objective function to guarantee $q_{1 i}^{x} q_{2 i}^{x}=0$ without modifying the optimal solution (where $\varepsilon$ is the "non-Archimedean" infinitesimal quantity).

The application of the same procedure to good and bad outputs (for good outputs there is a minor difference because the constraint is of type greater than or equal, but the procedure is very similar), the deterministic linear equivalent problem to be solved is:

$\operatorname{Max} \xi-\varepsilon\left(\sum_{i=1}^{m}\left(q_{1 i}^{x}+q_{2 i}^{x}\right)+\sum_{r=1}^{s}\left(q_{1 r}^{g}+q_{2 r}^{g}\right)+\sum_{f=1}^{h}\left(q_{1 f}^{b}+q_{2 f}^{b}\right)\right)$

s.t.

$\sum_{j=1}^{n} \mu_{i j}^{x} \lambda_{j}+\phi^{-1}(1-\alpha)\left(q_{1 i}^{x}+q_{2 i}^{x}\right) \leq \mu_{i k}^{x}$

$-\sigma_{i k}^{x}+\sum_{j=1}^{n} \sigma_{i j}^{x} \lambda_{j}=q_{1 i}^{x}-q_{2 i}^{x}$ $i=1, \ldots, m$

$q_{1 i}^{x} \geq 0, \quad q_{2 i}^{x} \geq 0$

$\sum_{j=1}^{n} \mu_{r j}^{g} \lambda_{j}-\phi^{-1}(1-\alpha)\left(q_{1 r}^{g}+q_{2 r}^{g}\right) \geq(1+\xi) \mu_{r k}^{g}$

$-(1+\xi) \sigma_{r k}^{g}+\sum_{j=1}^{n} \sigma_{r j}^{g} \lambda_{j}=q_{1 r}^{g}-q_{2 r}^{g}$ $r=1, \ldots, s$

$q_{1 r}^{g} \geq 0, \quad q_{2 r}^{g} \geq 0$

$\sum_{j=1}^{n} \mu_{f j}^{b} \lambda_{j}+\phi^{-1}(1-\alpha)\left(q_{1 f}^{b}+q_{2 f}^{b}\right) \leq(1-\xi) \mu_{f k}^{b}$

$-(1-\xi) \sigma_{f k}^{b}+\sum_{j=1}^{n} \sigma_{f j}^{b} \lambda_{j}=q_{1 f}^{b}-q_{2 f}^{b}$

$q_{1 f}^{b} \geq 0, \quad q_{2 f}^{b} \geq 0$

\section{$\xi$ Unrestricted}

The deterministic equivalent problem depends on $\mu_{i j}^{x}, \sigma_{i j}^{x}, \mu_{r j}^{g}$, $\sigma_{r j}^{g}, \mu_{f j}^{b}, \sigma_{f j}^{b}$. We may estimate these quantities in the unusual 
case in which we are able to replicate the data in similar conditions, and supposing that the frontier remains constant for the different replications. Notwithstanding, we usually have only one replication and then we have to select values for the unknown parameters. The natural hypothesis for means is $\mu_{i j}^{x}=x_{i j}, \mu_{r j}^{g}=g_{r j}$, $\mu_{f j}^{b}=b_{f j}$. The standard deviations must be fixed by experts taking into account the possible magnitude of random variations in the data under consideration [46].

For Managerial DEA, the procedure is similar. Only the input restrictions are modified and become:

$\left.\begin{array}{l}\sum_{j=1}^{n} \mu_{i j}^{x} \lambda_{j}-\phi^{-1}(1-\alpha)\left(q_{1 i}^{x}+q_{2 i}^{x}\right) \geq \mu_{i k}^{x} \\ -\sigma_{i k}^{x}+\sum_{j=1}^{n} \sigma_{i j}^{x} \lambda_{j}=q_{1 i}^{x}-q_{2 i}^{x} \\ q_{1 i}^{x} \geq 0, \quad q_{2 i}^{x} \geq 0\end{array}\right\} i=1, \ldots, m$

\section{A.3. Bayesian DEA.}

In Bayesian inference, the uncertainty related to unknown parameters in a statistical model is handled by the assumption of an existing distribution for it. By combining this prior function with the likelihood function via the Bayes theorem, we obtain the posterior distribution of the unknown parameters (conditional on the observed data), and this posterior distribution allows the obtention of inferences about both the unknown parameters and any function of them.

Usually, the posterior distribution is highly complex and analytical evaluation is not available. Therefore, the application of simulation-based approaches, such as the Gibbs sampling algorithm, [53-55] becomes necessary. It is important to remark that the Bayesian approach does not require the utilisation of the hypothetical large-sample constructs used in sampling-theory treatments, such as Bootstrap approach. The results of Bayesian inference are exact for finite samples, apart from simulation error.

We consider the inputs (for outputs the described process is similar) in order to specify the prior function and to obtain the posterior distribution. For simplicity in the notation, we will eliminate the upper script " $x$ ". As aforementioned, our deterministic-equivalent problem depends on $\mu_{i j}$ and $\sigma_{i j}$, but not on $x_{i j}$. Hence, the assumption of the hypothesis of inputs having a common mean is very restrictive, because in this case all the DMUs would have the same values of inputs in the deterministic equivalent problem. Consequently, we will consider $E\left[x_{i j}\right]=\mu_{i j}$.

If replicated data is available, $x_{i j} \sim N\left(\mu_{i j}, \sigma_{i j}^{2}\right)$ may be assumed, which enables us to apply the Bayesian framework for Normal distribution using a non-informative prior distribution [56]. In the usual case (not replicated data), the introduction of prior information about the standard deviations is required, since there is not such information available in the data.

First, we may assume a common variance for each input, that is, $x_{i j} \sim N\left(\mu_{i j}, \sigma_{i}^{2}\right)$, and second, we suppose a uniform distribution for the variance. The necessary information for $\sigma_{i}^{2}$ is not a single value, but a range of values representing variations of it.
Specifically, the prior distribution is $\sigma_{i}^{2} \sim U\left(\sigma_{i 0}^{2}, \sigma_{i 1}^{2}\right)$, where $U(a$, $b$ ) represents a uniform distribution in the interval $(a, b) . \sigma_{i 0}$ and $\sigma_{i 1}$ are to be fixed by experts which take into account the possible magnitude of random variations in the data. For the means, we assume the usual non-informative Jeffreys prior distribution $\pi\left(\mu_{i j}\right) \propto 1$ [57] and the prior distribution becomes:

$\pi\left(\mu_{i j}, \sigma_{i}^{2}\right)=\frac{1}{\sigma_{i 1}^{2}-\sigma_{i 0}^{2}}, \quad \sigma_{i 0}^{2}<\sigma_{i}^{2}<\sigma_{i 1}^{2}$

Taking into account that the Normal likelihood function is

$L\left(x_{i 1}, \ldots, x_{i n} \mid \mu_{i j}, \sigma_{i}^{2}\right) \propto \frac{1}{\sigma_{i}^{n}} \exp \left(-\frac{1}{2 \sigma_{i}^{2}} \sum_{j=1}^{n}\left(x_{i j}-\mu_{i j}\right)^{2}\right)$

where the symbol $\propto$ means "proportional to", the obtention of the posterior distribution is straightforward:

$\pi\left(\mu_{i j}, \sigma_{i}^{2} \mid x_{i j}\right) \propto \frac{1}{\sigma_{i}^{n}} \exp \left(-\frac{1}{2 \sigma_{i}^{2}} \sum_{j=1}^{n}\left(x_{i j}-\mu_{i j}\right)^{2}\right), \quad \sigma_{i 0}^{2}<\sigma_{i}^{2}<\sigma_{i 1}^{2}$

From this posterior distribution, we may apply the Gibbs algorithm by obtaining samples from the one-dimensional conditional distributions, that is, from $\sigma_{i}^{2} \mid \mu_{i j} x_{i j}$ and $\mu_{i j} \mid \mu_{i(j)}, \sigma_{i}^{2}, x_{i j}$ where $\mu_{i(j)}=\left(\mu_{i 1}, \ldots, \mu_{i j-1}, \mu_{i j+1}, \ldots, \mu_{i n}\right)$, because we obtain in both cases well-known distributions models and we are able to obtain simulated samples from them. For the variance, the posterior conditional distribution is:

$\pi\left(\sigma_{i}^{2} \mid \mu_{i j}, x_{i j}\right) \propto\left(\frac{1}{\sigma_{i}^{2}}\right)^{n / 2} \exp \left(-\frac{1}{2 \sigma_{i}^{2}} \sum_{j=1}^{n}\left(x_{i j}-\mu_{i j}\right)^{2}\right)$,

$\sigma_{i 0}^{2}<\sigma_{i}^{2}<\sigma_{i 1}^{2}$

and therefore $\sigma_{i}^{2} \mid \mu_{i j}, x_{i j} \sim \operatorname{IG}\left(\frac{n-2}{2}, \frac{1}{2} \sum_{j=1}^{n}\left(x_{i j}-\mu_{i j}\right)^{2}\right)$ truncated at the interval $\sigma_{i 0}^{2}<\sigma_{i}^{2}<\sigma_{i 1}^{2}$ where $X \sim \operatorname{IG}(\alpha, \beta)$ represents that $\mathrm{X}$ follows an Inverse-Gamma distribution with shape parameter $\alpha$ and scale parameter $\beta$ whose density function is:

$f(x)=\frac{\exp \left(-\frac{\beta}{x}\right)\left(\frac{\beta}{x}\right)^{\alpha}}{x \Gamma(\alpha)} \propto \exp \left(-\frac{\beta}{x}\right)\left(\frac{1}{x}\right)^{\alpha+1}$

For the unidimensional mean, the posterior conditional distribution is:

$\mu_{i j} \mid \mu_{i(j)}, \sigma_{i}^{2}, x_{i j} \sim N\left(x_{i j}, \sigma_{i}^{2}\right)$.

Applying the described Gibbs algorithm to inputs, good outputs and bad outputs, we obtain a large sample from the posterior distributions of $\mu_{i j}^{x}, \sigma_{i j}^{x}, \mu_{i j}^{g}, \sigma_{i j}^{g}, \mu_{i j}^{b}, \sigma_{i j}^{b}$. Solving the deterministic equivalent problems, we obtain a large sample from the posterior distributions of the efficiencies of the DMUs and then we can estimate the efficiencies and the Monte Carlo Methods may be applied to retrieve the inferences about them.

\section{Appendix B. RAW DEA results}




\begin{tabular}{|c|c|c|c|c|c|c|c|c|c|c|c|c|c|c|c|c|c|c|c|}
\hline & Architecture & Extractor & Resolution & $\begin{array}{l}\# \\
\text { Proposals }\end{array}$ & $\mathrm{mAP}$ & $\begin{array}{l}\text { mAP } \\
\text { (large) }\end{array}$ & $\begin{array}{l}\text { mAP } \\
\text { (medium) }\end{array}$ & $\begin{array}{l}\mathrm{mAP} \\
\text { (small) }\end{array}$ & $\begin{array}{l}\text { mAP @. } \\
75 I O U\end{array}$ & $\begin{array}{l}\text { mAP @. } \\
50 I O U\end{array}$ & $\begin{array}{l}\text { \# model } \\
\text { params }\end{array}$ & Memory & $\begin{array}{l}\text { Memory } \\
\text { std }\end{array}$ & CPU & $\begin{array}{l}\text { CPU } \\
\text { std }\end{array}$ & GPU & $\begin{array}{l}\text { GPU } \\
\text { std }\end{array}$ & FLOPS & DEA result \\
\hline 1 & Faster RCNN & VGG & 300 & 300 & 22,9 & 37,7 & 16,4 & 2,4 & 22,9 & 41,1 & 13,851 & 1699 & 81 & 1286 & 27 & 201 & 16 & 6432 & $0,992,863,189$ \\
\hline 2 & Faster RCNN & VGG & 300 & 10 & 19,6 & 32,8 & 12,7 & 1,7 & 20,4 & 33,4 & 13,851 & 1703 & 30 & 1322 & 41 & 203 & 16 & 2912 & 1 \\
\hline 3 & Faster RCNN & VGG & 300 & 20 & 21,5 & 35,7 & 14,8 & 2,1 & 21,9 & 37,6 & 13,851 & 1870 & 183 & 1261 & 5 & 204 & 15 & 3033 & 1 \\
\hline 4 & Faster RCNN & VGG & 300 & 50 & 22,5 & 37 & 16,1 & 2,3 & 22,5 & 40,2 & 13,851 & 1842 & 258 & 1280 & 41 & 202 & 15 & 3397 & $0,994,721,065$ \\
\hline 5 & Faster RCNN & VGG & 300 & 100 & 22,9 & 37,5 & 16,4 & 2,5 & 22,8 & 41 & 13,851 & 1713 & 152 & 1272 & 8 & 201 & 17 & 4004 & 1 \\
\hline 6 & Faster RCNN & VGG & 600 & 300 & 25,7 & 37,2 & 23 & 5,6 & 25,7 & 46 & 13,851 & 2048 & 210 & 1668 & 71 & 247 & 14 & 14,959 & $0,936,694,152$ \\
\hline 7 & Faster RCNN & VGG & 600 & 10 & 19 & 28,3 & 15,6 & 3,7 & 20,1 & 32,2 & 13,851 & 1853 & 388 & 1272 & 7 & 206 & 15 & 11,439 & 1 \\
\hline 8 & Faster RCNN & VGG & 600 & 20 & 22,3 & 32,8 & 19,3 & 5 & 23,1 & 38,7 & 13,851 & 1984 & 182 & 1282 & 5 & 205 & 16 & 1156 & 1 \\
\hline 9 & Faster RCNN & VGG & 600 & 50 & 24,8 & 35,6 & 22,2 & 5,9 & 25,3 & 44 & 13,851 & 1822 & 29 & 1327 & 6 & 209 & 16 & 11,925 & 1 \\
\hline 10 & Faster RCNN & VGG & 600 & 100 & 25,6 & 36,5 & 22,9 & 6,2 & 25,8 & 45,6 & 13,851 & 1948 & 210 & 1384 & 11 & 217 & 15 & 12,532 & 1 \\
\hline 11 & Faster RCNN & Resnet 101 & 300 & 300 & 26,5 & 43,5 & 19 & 3 & 27,2 & 44,6 & 6315 & 3236 & 190 & 2484 & 28 & 206 & 14 & 23,942 & $0,691,020,311$ \\
\hline 12 & Faster RCNN & Resnet 101 & 300 & 10 & 23,1 & 39,3 & 14,8 & 2,3 & 24,6 & 37,1 & 6315 & 560 & 19 & 355 & 13 & 92 & 13 & 2682 & 1 \\
\hline 13 & Faster RCNN & Resnet 101 & 300 & 20 & 24,8 & 41,6 & 17,1 & 2,6 & 26,1 & 40,9 & 6315 & 664 & 40 & 427 & 19 & 102 & 15 & 3415 & $0,990,104,601$ \\
\hline 14 & Faster RCNN & Resnet 101 & 300 & 50 & 25,8 & 42,7 & 18,4 & 2,9 & 26,7 & 43,4 & 6315 & 1021 & 10 & 634 & 15 & 112 & 17 & 5614 & 1 \\
\hline 15 & Faster RCNN & Resnet 101 & 300 & 100 & 26,3 & 43,2 & 18,8 & 3,1 & 27 & 44,3 & 6315 & 1459 & 33 & 1029 & 12 & 136 & 14 & 92,8 & $0,902,364,702$ \\
\hline 16 & Faster RCNN & Resnet 101 & 600 & 300 & 32,2 & 47,1 & 28,7 & 7,5 & 33,9 & 52,6 & 6315 & 4681 & 209 & 3127 & 17 & 248 & 14 & 29,661 & 1 \\
\hline 17 & Faster RCNN & Resnet 101 & 600 & 10 & 26,3 & 40 & 21,6 & 4,6 & 29,1 & 40,6 & 6315 & 1884 & 132 & 967 & 6 & 115 & 15 & 8402 & 1 \\
\hline 18 & Faster RCNN & Resnet 101 & 600 & 20 & 29,4 & 44,3 & 25,3 & 6,2 & 31,8 & 46,4 & 6315 & 1985 & 81 & 1085 & 93 & 126 & 16 & 9135 & 1 \\
\hline 19 & Faster RCNN & Resnet 101 & 600 & 50 & 31,3 & 46,1 & 27,6 & 7,2 & 33,4 & 50,6 & 6315 & 2267 & 106 & 1287 & 46 & 140 & 17 & 11,334 & 1 \\
\hline 20 & Faster RCNN & Resnet 101 & 600 & 100 & 32 & 46,7 & 28,5 & 7,5 & 33,9 & 52 & 6315 & 2756 & 138 & 1652 & 47 & 164 & 17 & 150 & $0,945,580,267$ \\
\hline 21 & Faster RCNN & Resnet 101 & 300 & 300 & 29,5 & 46,1 & 23,4 & 5,2 & 31,1 & 48,3 & 6315 & 4271 & 262 & 2930 & 23 & 233 & 15 & 28,999 & $0,821,391,556$ \\
\hline 22 & Faster RCNN & Resnet 101 & 300 & 10 & 24,5 & 41,1 & 16,6 & 3,5 & 27 & 37,9 & 6315 & 1378 & 44 & 789 & 23 & 108 & 13 & 7739 & $0,975,277,232$ \\
\hline 23 & Faster RCNN & Resnet 101 & 300 & 20 & 26,9 & 43,9 & 19,9 & 4,3 & 29,1 & 42,7 & 6315 & 1464 & 49 & 875 & 10 & 120 & 16 & 8472 & 1 \\
\hline 24 & Faster RCNN & Resnet 101 & 300 & 50 & 28,7 & 45,4 & 22,3 & 4,9 & 30,6 & 46,5 & 6315 & 1703 & 42 & 1098 & 8 & 132 & 18 & 10,671 & 1 \\
\hline 25 & Faster RCNN & Resnet 101 & 300 & 100 & 29,2 & 45,8 & 23,1 & 5,2 & 30,9 & 47,8 & 6315 & 2218 & 162 & 1502 & 12 & 161 & 15 & 14,337 & $0,937,373,734$ \\
\hline 26 & Faster RCNN & Resnet 101 & 600 & 300 & 34,4 & 47,7 & 31,8 & 10,7 & 37,1 & 54,6 & 6315 & 8043 & 301 & 4859 & 37 & 390 & 15 & 49,247 & $0,950,404,967$ \\
\hline 27 & Faster RCNN & Resnet 101 & 600 & 10 & 26,7 & 40,7 & 22,2 & 5,1 & 29,6 & 40,2 & 6315 & 5056 & 35 & 2672 & 34 & 196 & 16 & 27,987 & $0,792,525,311$ \\
\hline 28 & Faster RCNN & Resnet 101 & 600 & 20 & 30 & 44,6 & 26,8 & 6,9 & 33 & 46,2 & 6315 & 5146 & 143 & 2729 & 44 & 214 & 15 & 28,721 & $0,806,356,447$ \\
\hline 29 & Faster RCNN & Resnet 101 & 600 & 50 & 32,7 & 46,6 & 29,9 & 8,9 & 35,5 & 51,2 & 6315 & 5375 & 79 & 3047 & 113 & 234 & 16 & 3092 & $0,834,281,018$ \\
\hline 30 & Faster RCNN & Resnet 101 & 600 & 100 & 33,7 & 47,4 & 31,1 & 9,9 & 36,5 & 53,4 & 6315 & 5518 & 135 & 3384 & 65 & 267 & 16 & 34,585 & $0,861,698,229$ \\
\hline 31 & Faster RCNN & Inception V2 & 300 & 300 & 15,4 & 27,4 & 7,7 & 0,5 & 14,8 & 29 & 1331 & 2370 & 51 & 1388 & 5 & 178 & 15 & 11,822 & $0,873,408,819$ \\
\hline 32 & Faster RCNN & Inception V2 & 300 & 10 & 13,3 & 24,3 & 5,2 & 0,3 & 13,4 & 23,7 & 1331 & 211 & 6 & 153 & 7 & 79 & 13 & 7,63 & $0,952,521,136$ \\
\hline 33 & Faster RCNN & Inception V2 & 300 & 20 & 14,3 & 26 & 6,3 & 0,3 & 14,1 & 26,3 & 1331 & 280 & 11 & 198 & 6 & 90 & 13 & 1144 & $0,831,436,862$ \\
\hline 34 & Faster RCNN & Inception V2 & 300 & 50 & 15 & 26,9 & 7,2 & 0,5 & 14,5 & 28 & 1331 & 495 & 21 & 327 & 7 & 99 & 17 & 2288 & $0,764,144,374$ \\
\hline 35 & Faster RCNN & Inception V2 & 300 & 100 & 15,3 & 27,3 & 7,4 & 0,5 & 14,7 & 28,5 & 1331 & 861 & 51 & 535 & 7 & 118 & 15 & 4195 & $0,709,397,299$ \\
\hline 36 & Faster RCNN & Inception V2 & 600 & 300 & 21,9 & 34,4 & 17,6 & 3,1 & 21,8 & 39,5 & 1331 & 2596 & 63 & 1611 & 21 & 206 & 15 & 12,957 & $0,697,367,529$ \\
\hline 37 & Faster RCNN & Inception V2 & 600 & 10 & 16,4 & 27,3 & 10,7 & 1,9 & 17,2 & 28 & 1331 & 572 & 11 & 383 & 7 & 82 & 12 & 1898 & $0,925,119,313$ \\
\hline 38 & Faster RCNN & Inception V2 & 600 & 20 & 19 & 31,4 & 13,6 & 2,2 & 19,6 & 33,3 & 1331 & 669 & 16 & 446 & 22 & 98 & 13 & 2279 & $088,348,797$ \\
\hline 39 & Faster RCNN & Inception V2 & 600 & 50 & 20,8 & 33,5 & 16,1 & 2,5 & 21 & 37,2 & 1331 & 871 & 17 & 574 & 22 & 116 & 15 & 3423 & $0,840,390,039$ \\
\hline 40 & Faster RCNN & Inception V2 & 600 & 100 & 21,5 & 34 & 16,9 & 2,8 & 21,5 & 38,7 & 1331 & 1152 & 42 & 769 & 16 & 104 & 4 & 53,3 & 1 \\
\hline 41 & Faster RCNN & Inception V3 & 300 & 300 & 23,6 & 40,6 & 15,1 & 1,6 & 23,5 & 41,4 & 2627 & 5016 & 128 & 3214 & 18 & 353 & 14 & 3066 & $0,622,920,627$ \\
\hline 42 & Faster RCNN & Inception V3 & 300 & 10 & 20,2 & 36,1 & 11,1 & 1,3 & 21,3 & 33,7 & 2627 & 337 & 5 & 252 & 9 & 101 & 13 & 1577 & 1 \\
\hline 43 & Faster RCNN & Inception V3 & 300 & 20 & 21,9 & 38,4 & 12,9 & 1,6 & 22,6 & 37,3 & 2627 & 487 & 13 & 395 & 30 & 109 & 13 & 25,8 & $0,892,216,604$ \\
\hline 44 & Faster RCNN & Inception V3 & 300 & 50 & 23 & 39,8 & 14,4 & 1,7 & 23,2 & 40,1 & 2627 & 1017 & 40 & 669 & 11 & 137 & 13 & 5589 & $0,809,913,591$ \\
\hline 45 & Faster RCNN & Inception V3 & 300 & 100 & 23,4 & 40,3 & 14,9 & 1,7 & 23,4 & 41 & 2627 & 1734 & 106 & 1188 & 22 & 181 & 14 & 10,603 & $0,670,253,151$ \\
\hline 46 & Faster RCNN & Inception V3 & 600 & 300 & 29,6 & 44,9 & 25 & 5,3 & 30,6 & 50,2 & 2627 & 5386 & 143 & 3662 & 153 & 385 & 13 & 32,509 & $0,628,050,424$ \\
\hline 47 & Faster RCNN & Inception V3 & 600 & 10 & 23,9 & 38,5 & 17,4 & 3,2 & 25,9 & 38,1 & 2627 & 799 & 71 & 525 & 18 & 116 & 12 & 3426 & $0,936,410,729$ \\
\hline 48 & Faster RCNN & Inception V3 & 600 & 20 & 26,6 & 41,9 & 21 & 4,2 & 28,3 & 43,6 & 2627 & 1014 & 21 & 613 & 11 & 130 & 13 & 4429 & $0,959,575,307$ \\
\hline 49 & Faster RCNN & Inception V3 & 600 & 50 & 28,6 & 44 & 23,5 & 4,9 & 29,9 & 47,9 & 2627 & 1522 & 14 & 1048 & 60 & 153 & 16 & 7437 & $0,901,848,556$ \\
\hline 50 & Faster RCNN & Inception V3 & 600 & 100 & 29,2 & 44,6 & 24,3 & 5,4 & 30,2 & 49,4 & 2627 & 2293 & 81 & 1482 & 5 & 206 & 13 & 12,451 & 1 \\
\hline 51 & Faster RCNN & Inception Resnet V2 & 300 & 300 & 28,4 & 47 & 20,5 & 3,6 & 29,3 & 47,1 & 6002 & 14,769 & 103 & 7118 & 104 & 625 & 15 & 63,998 & $0,584,122,913$ \\
\hline 52 & Faster RCNN & Inception Resnet V2 & 300 & 10 & 24,7 & 42,7 & 15,6 & 2,8 & 26,6 & 38,8 & 6002 & 903 & 14 & 518 & 14 & 130 & 14 & 3345 & 1 \\
\hline
\end{tabular}




\begin{tabular}{|c|c|c|c|c|c|c|c|c|c|c|c|c|c|c|c|c|c|c|c|}
\hline & Architecture & Extractor & Resolution & $\begin{array}{l}\# \\
\text { Proposals }\end{array}$ & mAP & $\begin{array}{l}\mathrm{mAP} \\
\text { (large) }\end{array}$ & $\begin{array}{l}\text { mAP } \\
\text { (medium) }\end{array}$ & $\begin{array}{l}\text { mAP } \\
\text { (small) }\end{array}$ & $\begin{array}{l}\text { mAP @. } \\
75 I O U\end{array}$ & $\begin{array}{l}\text { mAP @. } \\
50 I O U\end{array}$ & $\begin{array}{l}\text { \# model } \\
\text { params }\end{array}$ & Memory & $\begin{array}{l}\text { Memory } \\
\text { std }\end{array}$ & $\mathrm{CPU}$ & $\begin{array}{l}\text { CPU } \\
\text { std }\end{array}$ & GPU & $\begin{array}{l}\text { GPU } \\
\text { std }\end{array}$ & FLOPS & DEA result \\
\hline 53 & Faster RCNN & Inception Resnet V2 & 300 & 20 & 26,6 & 45 & 18,1 & 3,4 & 28,1 & 43 & 6002 & 1295 & 36 & 756 & 19 & 147 & 13 & 5436 & $08,609,229$ \\
\hline 54 & Faster RCNN & Inception Resnet V2 & 300 & 50 & 27,9 & 46,3 & 19,9 & 3,7 & 29 & 45,9 & 6002 & 2622 & 69 & 1467 & 23 & 197 & 13 & 11,711 & $0,767,276,493$ \\
\hline 55 & Faster RCNN & Inception Resnet V2 & 300 & 100 & 28,2 & 46,7 & 20,3 & 3,7 & 28,2 & 46,7 & 6002 & 4908 & 212 & 2670 & 16 & 283 & 13 & 22,168 & $0,731,036,561$ \\
\hline 56 & Faster RCNN & Inception Resnet V2 & 600 & 300 & 35,4 & 52,8 & 31,1 & 8,3 & 37,5 & 56,3 & 6002 & 15,799 & 431 & 7652 & 31 & 671 & 13 & 68,043 & $0,920,287,958$ \\
\hline 57 & Faster RCNN & Inception Resnet V2 & 600 & 10 & 29 & 46,1 & 22,9 & 5,4 & 31,8 & 43,5 & 6002 & 2173 & 57 & 1138 & 28 & 157 & 14 & 73,9 & 09,074,608 \\
\hline 58 & Faster RCNN & Inception Resnet V2 & 600 & 20 & 32,2 & 49,6 & 27,1 & 6,7 & 34,9 & 49,6 & 6002 & 2651 & 50 & 1297 & 33 & 177 & 14 & 9481 & $0,923,089,298$ \\
\hline 59 & Faster RCNN & Inception Resnet V2 & 600 & 50 & 34,2 & 51,5 & 29,7 & 7,8 & 36,6 & 54,1 & 6002 & 4172 & 119 & 2058 & 62 & 232 & 13 & 15,756 & $0,816,011,426$ \\
\hline 60 & Faster RCNN & Inception Resnet V2 & 600 & 100 & 34,9 & 52,4 & 30,5 & 8,1 & 37,1 & 55,4 & 6002 & 6222 & 257 & 3390 & 47 & 319 & 14 & 26,213 & $0,792,351,318$ \\
\hline 61 & Faster RCNN & Inception Resnet V2 & 300 & 300 & 29,8 & 48,4 & 22,8 & 4,5 & 30,9 & 49,2 & 6002 & 6053 & 267 & 2905 & 66 & 272 & 19 & 66,545 & $0,638,583,584$ \\
\hline 62 & Faster RCNN & Inception Resnet V2 & 300 & 10 & 25,1 & 43,5 & 16,2 & 2,7 & 27,2 & 39 & 6002 & 6207 & 79 & 2937 & 31 & 287 & 15 & 5892 & $0,627,859,672$ \\
\hline 63 & Faster RCNN & Inception Resnet V2 & 300 & 20 & 27,4 & 46,2 & 19,3 & 3,8 & 29,4 & 43,7 & 6002 & 6138 & 109 & 2917 & 24 & 286 & 16 & 7984 & $0,663,382,971$ \\
\hline 64 & Faster RCNN & Inception Resnet V2 & 300 & 50 & 28,9 & 47,7 & 21,3 & 4,4 & 30,4 & 47,1 & 6002 & 5920 & 177 & 3036 & 15 & 283 & 16 & 14,258 & $0,726,514,381$ \\
\hline 65 & Faster RCNN & Inception Resnet V2 & 300 & 100 & 29,5 & 48,1 & 22,1 & 4,5 & 30,7 & 48,5 & 6002 & 6141 & 81 & 2945 & 52 & 281 & 17 & 24,715 & $0,629,430,321$ \\
\hline 66 & Faster RCNN & Inception Resnet V2 & 600 & 300 & 35,7 & 52,5 & 32 & 8,9 & 38 & 56,5 & 6002 & 19,467 & 284 & 10,018 & 491 & 855 & 14 & 78,899 & $0,704,720,088$ \\
\hline 67 & Faster RCNN & Inception Resnet V2 & 600 & 10 & 29,1 & 46,5 & 23,6 & 4,9 & 31,9 & 43,3 & 6002 & 6008 & 111 & 2889 & 34 & 285 & 15 & 18,245 & $0,664,052,088$ \\
\hline 68 & Faster RCNN & Inception Resnet V2 & 600 & 20 & 32,2 & 49,8 & 27,5 & 6,6 & 34,9 & 49,2 & 6002 & 6536 & 186 & 3230 & 92 & 308 & 13 & 20,337 & $0,674,861,576$ \\
\hline 69 & Faster RCNN & Inception Resnet V2 & 600 & 50 & 34,4 & 51,6 & 30,4 & 7,9 & 36,9 & 53,8 & 6002 & 7854 & 19 & 3849 & 29 & 367 & 15 & 26,611 & $0,992,603,286$ \\
\hline 70 & Faster RCNN & Inception Resnet V2 & 600 & 100 & 35,3 & 52,1 & 31,5 & 8,5 & 37,6 & 55,6 & 6002 & 10,341 & 296 & 5132 & 38 & 465 & 14 & 37,069 & $080,771,235$ \\
\hline 71 & Faster RCNN & MobileNet & 300 & 300 & 16,4 & 27,8 & 9,9 & 1 & 15,5 & 31,5 & 6,06 & 1147 & 28 & 427 & 22 & 94 & 17 & 2523 & $0,876,872,748$ \\
\hline 72 & Faster RCNN & MobileNet & 300 & 10 & 14,4 & 25,3 & 7,3 & 0,8 & 14,2 & 26,5 & 6,06 & 143 & 5 & 100 & 5 & 54 & 7 & 2,55 & 1 \\
\hline 73 & Faster RCNN & MobileNet & 300 & 20 & 15,5 & 27 & 8,6 & 0,9 & 15 & 29,3 & 6,06 & 172 & 6 & 123 & 4 & 61 & 9 & 3,33 & 1 \\
\hline 74 & Faster RCNN & MobileNet & 300 & 50 & 16,2 & 27,7 & 9,5 & 1 & 15,3 & 31 & 6,06 & 284 & 4 & 146 & 4 & 72 & 14 & 5,67 & 1 \\
\hline 75 & Faster RCNN & MobileNet & 300 & 100 & 16,4 & 27,9 & 9,9 & 0,9 & 15,5 & 31,5 & 6,06 & 447 & 17 & 203 & 9 & 81 & 14 & 9,59 & $0,876,768,764$ \\
\hline 76 & Faster RCNN & MobileNet & 600 & 300 & 19,8 & 29,3 & 17,5 & 3,8 & 18,3 & 38,5 & 6,06 & 1412 & 68 & 610 & 8 & 121 & 16 & 30,5 & 1 \\
\hline 77 & Faster RCNN & MobileNet & 600 & 10 & 15,1 & 23,7 & 11,8 & 2,4 & 14,8 & 28 & 6,06 & 487 & 7 & 273 & 12 & 63 & 6 & 7,82 & 1 \\
\hline 78 & Faster RCNN & MobileNet & 600 & 20 & 17,4 & 26,8 & 14,4 & 2,8 & 16,4 & 33,2 & 6,06 & 507 & 4 & 292 & 2 & 69 & 9 & 8,6 & 1 \\
\hline 79 & Faster RCNN & MobileNet & 600 & 50 & 19,1 & 28,6 & 16,7 & 3,5 & 17,8 & 37 & 6,06 & 572 & 25 & 349 & 11 & 79 & 14 & 1094 & 1 \\
\hline 80 & Faster RCNN & MobileNet & 600 & 100 & 19,7 & 29,2 & 17,4 & 3,7 & 18,1 & 38,3 & 6,06 & 768 & 10 & 376 & 18 & 98 & 14 & 1485 & 1 \\
\hline 81 & R-FCN & Resnet 101 & 300 & 300 & 25,2 & 41,1 & 18,7 & 2,5 & 25,9 & 43,4 & 6848 & 992 & 30 & 444 & 15 & 103 & 6 & 2714 & 1 \\
\hline 82 & R-FCN & Resnet 101 & 300 & 100 & 25,2 & 41,2 & 18,6 & 2,6 & 26 & 43,5 & 6848 & 861 & 47 & 437 & 25 & 94 & 5 & 2714 & 1 \\
\hline 83 & R-FCN & Resnet 101 & 300 & 50 & 24,9 & 40,9 & 1821 & 2,5 & 25,8 & 42,7 & 6848 & 798 & 51 & 425 & 20 & 92 & 6 & 2714 & 1 \\
\hline 84 & R-FCN & Resnet 101 & 300 & 20 & 23,8 & 39,7 & 16,8 & 2,1 & 25,2 & 40,1 & 6848 & 713 & 79 & 413 & 10 & 90 & 6 & 2714 & 1 \\
\hline 85 & R-FCN & Resnet 101 & 300 & 10 & 22,1 & 37,6 & 14,4 & 1,8 & 23,9 & 36,3 & 6848 & 708 & 25 & 428 & 31 & 89 & 6 & 2714 & 1 \\
\hline 86 & R-FCN & Resnet 101 & 600 & 300 & 30,4 & 43,3 & 28,3 & 7,2 & 32 & 51,4 & 6848 & 2878 & 75 & 1536 & 42 & 161 & 8 & 1073 & 1 \\
\hline 87 & R-FCN & Resnet 101 & 600 & 100 & 30,5 & 43,4 & 28,5 & 7,4 & 32,1 & 51,6 & 6848 & 2651 & 19 & 1465 & 62 & 141 & 9 & 1073 & 1 \\
\hline 88 & R-FCN & Resnet 101 & 600 & 50 & 30,1 & 43,3 & 27,9 & 7,3 & 31,8 & 50,4 & 6848 & 2591 & 100 & 1419 & 40 & 134 & 8 & 1073 & 1 \\
\hline 89 & R-FCN & Resnet 101 & 600 & 20 & 28,3 & 41,3 & 25,7 & 6,2 & 30,4 & 46,5 & 6848 & 2600 & 69 & 1503 & 103 & 131 & 8 & 1073 & 1 \\
\hline 90 & R-FCN & Resnet 101 & 600 & 10 & 25,3 & 37,9 & 21,9 & 4,9 & 27,7 & 40,5 & 6848 & 2530 & 34 & 1438 & 64 & 131 & 8 & 1073 & $0,998,772,809$ \\
\hline 91 & R-FCN & Resnet 101 & 300 & 300 & 27,6 & 43,3 & 22,7 & 4,4 & 29 & 46,1 & 6848 & 2190 & 26 & 1330 & 25 & 147 & 7 & 10,067 & 1 \\
\hline 92 & R-FCN & Resnet 101 & 300 & 100 & 27,4 & 43,5 & 22,3 & 4,2 & 28,9 & 45,7 & 6848 & 2101 & 97 & 1314 & 21 & 133 & 6 & 10,067 & $0,970,523,273$ \\
\hline 93 & R-FCN & Resnet 101 & 300 & 50 & 27 & 43,3 & 21,6 & 3,7 & 28,7 & 44,5 & 6848 & 2072 & 86 & 1325 & 57 & 128 & 6 & 10,067 & $0,947,762,286$ \\
\hline 94 & R-FCN & Resnet 101 & 300 & 20 & 25,2 & 42 & 18,6 & 3 & 27,4 & 40,7 & 6848 & 2088 & 86 & 1303 & 23 & 127 & 6 & 10,067 & $0,927,504,965$ \\
\hline 95 & R-FCN & Resnet 101 & 300 & 10 & 23,1 & 39,4 & 15,7 & 2,4 & 25,5 & 36,5 & 6848 & 1943 & 93 & 1326 & 57 & 126 & 6 & 10,067 & $0,885,004,763$ \\
\hline 96 & R-FCN & Resnet 101 & 600 & 300 & 31,9 & 43 & 30,8 & 10,4 & 33,7 & 52,4 & 6848 & 7746 & 394 & 4823 & 145 & 389 & 14 & 39,178 & $084,314,076$ \\
\hline 97 & R-FCN & Resnet 101 & 600 & 100 & 31,7 & 43,2 & 30,7 & 10 & 33,8 & 52,2 & 6848 & 7143 & 97 & 4747 & 125 & 327 & 13 & 39,178 & $085,856,585$ \\
\hline 98 & R-FCN & Resnet 101 & 600 & 50 & 31,1 & 42,9 & 30,1 & 9,3 & 33,3 & 50,8 & 6848 & 7842 & 163 & 4769 & 108 & 313 & 12 & 39,178 & $0,849,016,854$ \\
\hline 99 & R-FCN & Resnet 101 & 600 & 20 & 28,8 & 41,7 & 26,9 & 7,2 & 31,5 & 46,1 & 6848 & 7562 & 251 & 4801 & 134 & 304 & 13 & 39,178 & $0,704,610,835$ \\
\hline 100 & R-FCN & Resnet 101 & 600 & 10 & 25,6 & 38,8 & 22,6 & 5,4 & 28,3 & 40,1 & 6848 & 7553 & 244 & 4685 & 113 & 302 & 13 & 39,178 & $0,591,162,527$ \\
\hline 101 & R-FCN & Inception V2 & 300 & 300 & 15,4 & 27,8 & 7 & 0 & 14,5 & 29,5 & 1806 & 286 & 14 & 144 & 7 & 72 & 5 & 5,09 & 1 \\
\hline 102 & R-FCN & Inception V2 & 300 & 100 & 15,5 & 27,9 & 7,2 & 1 & 14,6 & 29,9 & 1806 & 208 & 11 & 125 & 6 & 69 & 6 & 5,09 & 1 \\
\hline 103 & R-FCN & Inception V2 & 300 & 50 & 15,3 & 27,7 & 7,1 & 1 & 14,5 & 29,6 & 1806 & 202 & 13 & 125 & 6 & 66 & 6 & 5,09 & 1 \\
\hline
\end{tabular}




\begin{tabular}{|c|c|c|c|c|c|c|c|c|c|c|c|c|c|c|c|c|c|c|c|}
\hline & Architecture & Extractor & Resolution & $\begin{array}{l}\# \\
\text { Proposals }\end{array}$ & $\mathrm{mAP}$ & $\begin{array}{l}\mathrm{mAP} \\
\text { (large) }\end{array}$ & $\begin{array}{l}\mathrm{mAP} \\
\text { (medium) }\end{array}$ & $\begin{array}{l}\mathrm{mAP} \\
\text { (small) }\end{array}$ & $\begin{array}{l}\text { mAP @. } \\
75 I O U\end{array}$ & $\begin{array}{l}\text { mAP @. } \\
50 I O U\end{array}$ & $\begin{array}{l}\# \text { model } \\
\text { params }\end{array}$ & Memory & $\begin{array}{l}\text { Memory } \\
\text { std }\end{array}$ & $\mathrm{CPU}$ & $\begin{array}{l}\text { CPU } \\
\text { std }\end{array}$ & GPU & $\begin{array}{l}\text { GPU } \\
\text { std }\end{array}$ & FLOPS & DEA result \\
\hline 104 & R-FCN & Inception V2 & 300 & 20 & 14,7 & 26,8 & 6,5 & 0 & 14,1 & 27,9 & 1806 & 177 & 10 & 123 & 8 & 64 & 6 & 5,09 & 1 \\
\hline 105 & R-FCN & Inception V2 & 300 & 10 & 13,8 & 25,2 & 5,7 & 0 & 13,6 & 25,6 & 1806 & 164 & 12 & 121 & 7 & 66 & 6 & 5,09 & 1 \\
\hline 106 & R-FCN & Inception V2 & 600 & 300 & 19,8 & 31,8 & 15,2 & 1,8 & 18,2 & 38,3 & 1806 & 715 & 6 & 445 & 16 & 101 & 6 & 1985 & 1 \\
\hline 107 & R-FCN & Inception V2 & 600 & 100 & 20,1 & 31,9 & 15,6 & 2 & 18,5 & 39,1 & 1806 & 647 & 19 & 394 & 15 & 87 & 6 & 1985 & 1 \\
\hline 108 & R-FCN & Inception V2 & 600 & 50 & 19,7 & 31,6 & 15 & 2 & 18,3 & 38,1 & 1806 & 624 & 25 & 391 & 19 & 84 & 7 & 1985 & $0,974,865,874$ \\
\hline 109 & R-FCN & Inception V2 & 600 & 20 & 18,3 & 30,1 & 13,2 & 1,8 & 17,4 & 34,7 & 1806 & 609 & 9 & 378 & 5 & 81 & 6 & 1985 & 1 \\
\hline 110 & R-FCN & Inception V2 & 600 & 10 & 15,9 & 26,5 & 10,6 & 1,6 & 15,6 & 29,5 & 1806 & 581 & 12 & 376 & 8 & 79 & 7 & 1985 & $0,977,454,298$ \\
\hline 111 & R-FCN & Inception Resnet V2 & 300 & 300 & 22,5 & 39,6 & 13,2 & 1 & 22,7 & 39,8 & 6506 & 696 & 108 & 339 & 10 & 108 & 6 & 1499 & 1 \\
\hline 112 & R-FCN & Inception Resnet V2 & 300 & 100 & 22,8 & 39,8 & 13,8 & 1,1 & 23 & 40,7 & 6506 & 642 & 71 & 315 & 13 & 100 & 7 & 1499 & 1 \\
\hline 113 & R-FCN & Inception Resnet V2 & 300 & 50 & 22,8 & 39,6 & 14 & 1,2 & 23 & 40,6 & 6506 & 679 & 33 & 314 & 7 & 101 & 6 & 1499 & 1 \\
\hline 114 & R-FCN & Inception Resnet V2 & 300 & 20 & 22,3 & 38,9 & 13,5 & 1 & 22,8 & 39,1 & 6506 & 611 & 42 & 312 & 13 & 99 & 6 & 1499 & 1 \\
\hline 115 & R-FCN & Inception Resnet V2 & 300 & 10 & 21,1 & 37,5 & 12,1 & 1,1 & 22,1 & 36 & 6506 & 637 & 96 & 324 & 10 & 98 & 7 & 1499 & 1 \\
\hline 116 & R-FCN & Inception Resnet V2 & 600 & 300 & 30,7 & 46,2 & 26,8 & 5,6 & 31,7 & 52,3 & 6506 & 2173 & 108 & 1048 & 24 & 165 & 6 & 6396 & 1 \\
\hline 117 & R-FCN & Inception Resnet V2 & 600 & 100 & 30,9 & 46,4 & 27 & 6,1 & 31,9 & 52,8 & 6506 & 2121 & 36 & 993 & 26 & 150 & 6 & 6396 & 1 \\
\hline 118 & R-FCN & Inception Resnet V2 & 600 & 50 & 30,7 & 46,1 & 26,8 & 6,2 & 32 & 52,3 & 6506 & 2150 & 83 & 1060 & 41 & 146 & 6 & 6396 & 1 \\
\hline 119 & R-FCN & Inception Resnet V2 & 600 & 20 & 29,3 & 44,9 & 25 & 5,4 & 31,1 & 48,8 & 6506 & 2051 & 12 & 1007 & 30 & 144 & 6 & 6396 & 1 \\
\hline 120 & R-FCN & Inception Resnet V2 & 600 & 10 & 27,1 & 42,7 & 22,1 & 4,4 & 29,3 & 43,9 & 6506 & 2151 & 62 & 1034 & 12 & 141 & 5 & 6396 & 1 \\
\hline 121 & R-FCN & Inception Resnet V2 & 300 & 300 & 26 & 43,5 & 18,7 & 2 & 26,4 & 45,3 & 6506 & 1609 & 37 & 827 & 14 & 142 & 7 & 4774 & $0,967,072,717$ \\
\hline 122 & R-FCN & Inception Resnet V2 & 300 & 100 & 26 & 43,5 & 18,6 & 2,1 & 26,4 & 45,2 & 6506 & 1557 & 28 & 841 & 10 & 134 & 6 & 4774 & 1 \\
\hline 123 & R-FCN & Inception Resnet V2 & 300 & 50 & 25,6 & 43,2 & 17,9 & 1,9 & 26,1 & 44,3 & 6506 & 1564 & 14 & 808 & 5 & 133 & 7 & 4774 & 1 \\
\hline 124 & R-FCN & Inception Resnet V2 & 300 & 20 & 24,4 & 42,4 & 15,9 & 1,7 & 25,4 & 41,3 & 6506 & 1499 & 83 & 796 & 3 & 128 & 8 & 4774 & 1 \\
\hline 125 & R-FCN & Inception Resnet V2 & 300 & 10 & 22,5 & 40,1 & 13,5 & 1,5 & 23,9 & 37,4 & 6506 & 1501 & 102 & 791 & 14 & 131 & 6 & 4774 & $0,931,535,439$ \\
\hline 126 & R-FCN & Inception Resnet V2 & 600 & 300 & 32,3 & 46,9 & 29,3 & 8 & 33,8 & 53,9 & 6506 & 7289 & 46 & 3220 & 60 & 368 & 8 & 20,545 & 1 \\
\hline 127 & R-FCN & Inception Resnet V2 & 600 & 100 & 32,1 & 46,8 & 29,3 & 8 & 33,7 & 53,7 & 6506 & 6653 & 53 & 3319 & 70 & 328 & 6 & 20,545 & 1 \\
\hline 128 & R-FCN & Inception Resnet V2 & 600 & 50 & 31,5 & 46,4 & 28,6 & 7,6 & 33,3 & 52,2 & 6506 & 6771 & 258 & 3315 & 32 & 313 & 7 & 20,545 & 1 \\
\hline 129 & R-FCN & Inception Resnet V2 & 600 & 20 & 29,7 & 45,2 & 26,4 & 6,7 & 32 & 48,3 & 6506 & 6958 & 137 & 3318 & 100 & 302 & 7 & 20,545 & $0,897,209,934$ \\
\hline 130 & R-FCN & Inception Resnet V2 & 600 & 10 & 27,1 & 42,7 & 22,7 & 4,8 & 29,7 & 42,9 & 6506 & 7001 & 40 & 3320 & 21 & 299 & 7 & 20,545 & $0,934,269,357$ \\
\hline 131 & R-FCN & MobileNet & 300 & 300 & 15 & 26,3 & 7,6 & 0 & 13,7 & 29,4 & 10,8 & 246 & 4 & 114 & 4 & 66 & 5 & 2,44 & 1 \\
\hline 132 & R-FCN & MobileNet & 300 & 100 & 15,2 & 26,5 & 7,9 & 1 & 13,8 & 30 & 10,8 & 170 & 10 & 94 & 8 & 57 & 7 & 2,44 & 1 \\
\hline 133 & R-FCN & MobileNet & 300 & 50 & 15,2 & 26,5 & 7,8 & 1 & 13,8 & 30 & 10,8 & 159 & 10 & 92 & 8 & 60 & 5 & 2,44 & 1 \\
\hline 134 & R-FCN & MobileNet & 300 & 20 & 14,7 & 25,9 & 7,3 & 1 & 13,6 & 28,7 & 10,8 & 150 & 10 & 96 & 7 & 59 & 5 & 2,44 & 1 \\
\hline 135 & R-FCN & MobileNet & 300 & 10 & 13,8 & 24,4 & 6,5 & 1 & 13,1 & 26,4 & 10,8 & 137 & 11 & 97 & 5 & 58 & 5 & 2,44 & 1 \\
\hline 136 & R-FCN & MobileNet & 600 & 300 & 16,5 & 25,8 & 13,2 & 1,5 & 14 & 33,8 & 10,8 & 607 & 17 & 341 & 25 & 86 & 5 & 9,49 & 1 \\
\hline 137 & R-FCN & MobileNet & 600 & 100 & 17 & 26,2 & 13,8 & 1,8 & 14,3 & 35,1 & 10,8 & 537 & 6 & 296 & 8 & 73 & 6 & 9,49 & 1 \\
\hline 138 & R-FCN & MobileNet & 600 & 50 & 16,9 & 26 & 13,7 & 2 & 14,2 & 34,8 & 10,8 & 514 & 2 & 283 & 8 & 70 & 6 & 9,49 & 1 \\
\hline 139 & R-FCN & MobileNet & 600 & 20 & 15,9 & 24,9 & 12,6 & 1,8 & 13,7 & 32,3 & 10,8 & 511 & 15 & 285 & 8 & 69 & 5 & 9,49 & 1 \\
\hline 140 & R-FCN & MobileNet & 600 & 10 & 14,1 & 22,2 & 10,6 & 1,3 & 12,5 & 27,9 & 10,8 & 507 & 20 & 280 & 9 & 67 & 5 & 9,49 & 1 \\
\hline
\end{tabular}




\begin{tabular}{|c|c|c|c|c|c|c|c|c|c|c|c|c|c|c|c|c|c|c|c|}
\hline & Architecture & Extractor & Resolution & $\begin{array}{l}\# \\
\text { Proposals }\end{array}$ & mAP & $\begin{array}{l}\mathrm{mAP} \\
\text { (large) }\end{array}$ & $\begin{array}{l}\text { mAP } \\
\text { (medium) }\end{array}$ & $\begin{array}{l}\mathrm{mAP} \\
\text { (small) }\end{array}$ & $\begin{array}{l}\text { mAP @. } \\
75 I O U\end{array}$ & $\begin{array}{l}\text { mAP @. } \\
50 I O U\end{array}$ & $\begin{array}{l}\text { \# model } \\
\text { params }\end{array}$ & Memory & $\begin{array}{l}\text { Memory } \\
\text { std }\end{array}$ & CPU & $\begin{array}{l}\mathrm{CPU} \\
\text { std }\end{array}$ & GPU & $\begin{array}{l}\text { GPU } \\
\text { std }\end{array}$ & FLOPS & DEA result \\
\hline 1 & Faster RCNN & VGG & 300 & 300 & 22,9 & 37,7 & 16,4 & 2,4 & 22,9 & 41,1 & 13,851 & 1699 & 81 & 1286 & 27 & 201 & 16 & 6432 & 1 \\
\hline 2 & Faster RCNN & VGG & 300 & 10 & 19,6 & 32,8 & 12,7 & 1,7 & 20,4 & 33,4 & 13,851 & 1703 & 30 & 1322 & 41 & 203 & 16 & 2912 & 1 \\
\hline 3 & Faster RCNN & VGG & 300 & 20 & 21,5 & 35,7 & 14,8 & 2,1 & 21,9 & 37,6 & 13,851 & 1870 & 183 & 1261 & 5 & 204 & 15 & 3033 & 1 \\
\hline 4 & Faster RCNN & VGG & 300 & 50 & 22,5 & 37 & 16,1 & 2,3 & 22,5 & 40,2 & 13,851 & 1842 & 258 & 1280 & 41 & 202 & 15 & 3397 & 1 \\
\hline 5 & Faster RCNN & VGG & 300 & 100 & 22,9 & 37,5 & 16,4 & 2,5 & 22,8 & 41 & 13,851 & 1713 & 152 & 1272 & 8 & 201 & 17 & 4004 & 1 \\
\hline 6 & Faster RCNN & VGG & 600 & 300 & 25,7 & 37,2 & 23 & 5,6 & 25,7 & 46 & 13,851 & 2048 & 210 & 1668 & 71 & 247 & 14 & 14,959 & 1 \\
\hline 7 & Faster RCNN & VGG & 600 & 10 & 19 & 28,3 & 15,6 & 3,7 & 20,1 & 32,2 & 13,851 & 1853 & 388 & 1272 & 7 & 206 & 15 & 11,439 & 1 \\
\hline 8 & Faster RCNN & VGG & 600 & 20 & 22,3 & 32,8 & 19,3 & 5 & 23,1 & 38,7 & 13,851 & 1984 & 182 & 1282 & 5 & 205 & 16 & 1156 & 1 \\
\hline 9 & Faster RCNN & VGG & 600 & 50 & 24,8 & 35,6 & 22,2 & 5,9 & 25,3 & 44 & 13,851 & 1822 & 29 & 1327 & 6 & 209 & 16 & 11,925 & 1 \\
\hline 10 & Faster RCNN & VGG & 600 & 100 & 25,6 & 36,5 & 22,9 & 6,2 & 25,8 & 45,6 & 13,851 & 1948 & 210 & 1384 & 11 & 217 & 15 & 12,532 & 1 \\
\hline 11 & Faster RCNN & Resnet 101 & 300 & 300 & 26,5 & 43,5 & 19 & 3 & 27,2 & 44,6 & 6315 & 3236 & 190 & 2484 & 28 & 206 & 14 & 23,942 & $0,721,635,676$ \\
\hline 12 & Faster RCNN & Resnet 101 & 300 & 10 & 23,1 & 39,3 & 14,8 & 2,3 & 24,6 & 37,1 & 6315 & 560 & 19 & 355 & 13 & 92 & 13 & 2682 & 1 \\
\hline 13 & Faster RCNN & Resnet 101 & 300 & 20 & 24,8 & 41,6 & 17,1 & 2,6 & 26,1 & 40,9 & 6315 & 664 & 40 & 427 & 19 & 102 & 15 & 3415 & 1 \\
\hline 14 & Faster RCNN & Resnet 101 & 300 & 50 & 25,8 & 42,7 & 18,4 & 2,9 & 26,7 & 43,4 & 6315 & 1021 & 10 & 634 & 15 & 112 & 17 & 5614 & 1 \\
\hline 15 & Faster RCNN & Resnet 101 & 300 & 100 & 26,3 & 43,2 & 18,8 & 3,1 & 27 & 44,3 & 6315 & 1459 & 33 & 1029 & 12 & 136 & 14 & 92,8 & $0,932,975,719$ \\
\hline 16 & Faster RCNN & Resnet 101 & 600 & 300 & 32,2 & 47,1 & 28,7 & 7,5 & 33,9 & 52,6 & 6315 & 4681 & 209 & 3127 & 17 & 248 & 14 & 29,661 & 1 \\
\hline 17 & Faster RCNN & Resnet 101 & 600 & 10 & 26,3 & 40 & 21,6 & 4,6 & 29,1 & 40,6 & 6315 & 1884 & 132 & 967 & 6 & 115 & 15 & 8402 & 1 \\
\hline 18 & Faster RCNN & Resnet 101 & 600 & 20 & 29,4 & 44,3 & 25,3 & 6,2 & 31,8 & 46,4 & 6315 & 1985 & 81 & 1085 & 93 & 126 & 16 & 9135 & 1 \\
\hline 19 & Faster RCNN & Resnet 101 & 600 & 50 & 31,3 & 46,1 & 27,6 & 7,2 & 33,4 & 50,6 & 6315 & 2267 & 106 & 1287 & 46 & 140 & 17 & 11,334 & 1 \\
\hline 20 & Faster RCNN & Resnet 101 & 600 & 100 & 32 & 46,7 & 28,5 & 7,5 & 33,9 & 52 & 6315 & 2756 & 138 & 1652 & 47 & 164 & 17 & 150 & $097,035,316$ \\
\hline 21 & Faster RCNN & Resnet 101 & 300 & 300 & 29,5 & 46,1 & 23,4 & 5,2 & 31,1 & 48,3 & 6315 & 4271 & 262 & 2930 & 23 & 233 & 15 & 28,999 & $0,856,038,972$ \\
\hline 22 & Faster RCNN & Resnet 101 & 300 & 10 & 24,5 & 41,1 & 16,6 & 3,5 & 27 & 37,9 & 6315 & 1378 & 44 & 789 & 23 & 108 & 13 & 7739 & $0,979,869,385$ \\
\hline 23 & Faster RCNN & Resnet 101 & 300 & 20 & 26,9 & 43,9 & 19,9 & 4,3 & 29,1 & 42,7 & 6315 & 1464 & 49 & 875 & 10 & 120 & 16 & 8472 & 1 \\
\hline 24 & Faster RCNN & Resnet 101 & 300 & 50 & 28,7 & 45,4 & 22,3 & 4,9 & 30,6 & 46,5 & 6315 & 1703 & 42 & 1098 & 8 & 132 & 18 & 10,671 & 1 \\
\hline 25 & Faster RCNN & Resnet 101 & 300 & 100 & 29,2 & 45,8 & 23,1 & 5,2 & 30,9 & 47,8 & 6315 & 2218 & 162 & 1502 & 12 & 161 & 15 & 14,337 & $097,948,059$ \\
\hline 26 & Faster RCNN & Resnet 101 & 600 & 300 & 34,4 & 47,7 & 31,8 & 10,7 & 37,1 & 54,6 & 6315 & 8043 & 301 & 4859 & 37 & 390 & 15 & 49,247 & 1 \\
\hline 27 & Faster RCNN & Resnet 101 & 600 & 10 & 26,7 & 40,7 & 22,2 & 5,1 & 29,6 & 40,2 & 6315 & 5056 & 35 & 2672 & 34 & 196 & 16 & 27,987 & $0,804,383,004$ \\
\hline 28 & Faster RCNN & Resnet 101 & 600 & 20 & 30 & 44,6 & 26,8 & 6,9 & 33 & 46,2 & 6315 & 5146 & 143 & 2729 & 44 & 214 & 15 & 28,721 & $0,837,371,399$ \\
\hline 29 & Faster RCNN & Resnet 101 & 600 & 50 & 32,7 & 46,6 & 29,9 & 8,9 & 35,5 & 51,2 & 6315 & 5375 & 79 & 3047 & 113 & 234 & 16 & 3092 & $0,940,473,942$ \\
\hline 30 & Faster RCNN & Resnet 101 & 600 & 100 & 33,7 & 47,4 & 31,1 & 9,9 & 36,5 & 53,4 & 6315 & 5518 & 135 & 3384 & 65 & 267 & 16 & 34,585 & 1 \\
\hline 31 & Faster RCNN & Inception V2 & 300 & 300 & 15,4 & 27,4 & 7,7 & 0,5 & 14,8 & 29 & 1331 & 2370 & 51 & 1388 & 5 & 178 & 15 & 11,822 & $0,878,056,912$ \\
\hline 32 & Faster RCNN & Inception V2 & 300 & 10 & 13,3 & 24,3 & 5,2 & 0,3 & 13,4 & 23,7 & 1331 & 211 & 6 & 153 & 7 & 79 & 13 & 7,63 & $0,969,136,168$ \\
\hline 33 & Faster RCNN & Inception V2 & 300 & 20 & 14,3 & 26 & 6,3 & 0,3 & 14,1 & 26,3 & 1331 & 280 & 11 & 198 & 6 & 90 & 13 & 1144 & $0,849,455,864$ \\
\hline 34 & Faster RCNN & Inception V2 & 300 & 50 & 15 & 26,9 & 7,2 & 0,5 & 14,5 & 28 & 1331 & 495 & 21 & 327 & 7 & 99 & 17 & 2288 & $0,770,753,102$ \\
\hline 35 & Faster RCNN & Inception V2 & 300 & 100 & 15,3 & 27,3 & 7,4 & 0,5 & 14,7 & 28,5 & 1331 & 861 & 51 & 535 & 7 & 118 & 15 & 4195 & $0,711,132,149$ \\
\hline 36 & Faster RCNN & Inception V2 & 600 & 300 & 21,9 & 34,4 & 17,6 & 3,1 & 21,8 & 39,5 & 1331 & 2596 & 63 & 1611 & 21 & 206 & 15 & 12,957 & $0,714,178,401$ \\
\hline 37 & Faster RCNN & Inception V2 & 600 & 10 & 16,4 & 27,3 & 10,7 & 1,9 & 17,2 & 28 & 1331 & 572 & 11 & 383 & 7 & 82 & 12 & 1898 & $0,930,786,594$ \\
\hline 38 & Faster RCNN & Inception V2 & 600 & 20 & 19 & 31,4 & 13,6 & 2,2 & 19,6 & 33,3 & 1331 & 669 & 16 & 446 & 22 & 98 & 13 & 2279 & $0,918,079,095$ \\
\hline 39 & Faster RCNN & Inception V2 & 600 & 50 & 20,8 & 33,5 & 16,1 & 2,5 & 21 & 37,2 & 1331 & 871 & 17 & 574 & 22 & 116 & 15 & 3423 & $0,856,791,909$ \\
\hline 40 & Faster RCNN & Inception V2 & 600 & 100 & 21,5 & 34 & 16,9 & 2,8 & 21,5 & 38,7 & 1331 & 1152 & 42 & 769 & 16 & 104 & 4 & 53,3 & 1 \\
\hline 41 & Faster RCNN & Inception V3 & 300 & 300 & 23,6 & 40,6 & 15,1 & 1,6 & 23,5 & 41,4 & 2627 & 5016 & 128 & 3214 & 18 & 353 & 14 & 3066 & $0,653,800,701$ \\
\hline 42 & Faster RCNN & Inception V3 & 300 & 10 & 20,2 & 36,1 & 11,1 & 1,3 & 21,3 & 33,7 & 2627 & 337 & 5 & 252 & 9 & 101 & 13 & 1577 & 1 \\
\hline 43 & Faster RCNN & Inception V3 & 300 & 20 & 21,9 & 38,4 & 12,9 & 1,6 & 22,6 & 37,3 & 2627 & 487 & 13 & 395 & 30 & 109 & 13 & 25,8 & $0,997,415,181$ \\
\hline 44 & Faster RCNN & Inception V3 & 300 & 50 & 23 & 39,8 & 14,4 & 1,7 & 23,2 & 40,1 & 2627 & 1017 & 40 & 669 & 11 & 137 & 13 & 5589 & $0,851,708,909$ \\
\hline 45 & Faster RCNN & Inception V3 & 300 & 100 & 23,4 & 40,3 & 14,9 & 1,7 & 23,4 & 41 & 2627 & 1734 & 106 & 1188 & 22 & 181 & 14 & 10,603 & $0,690,446,077$ \\
\hline 46 & Faster RCNN & Inception V3 & 600 & 300 & 29,6 & 44,9 & 25 & 5,3 & 30,6 & 50,2 & 2627 & 5386 & 143 & 3662 & 153 & 385 & 13 & 32,509 & $0,628,652,862$ \\
\hline 47 & Faster RCNN & Inception V3 & 600 & 10 & 23,9 & 38,5 & 17,4 & 3,2 & 25,9 & 38,1 & 2627 & 799 & 71 & 525 & 18 & 116 & 12 & 3426 & 1 \\
\hline 48 & Faster RCNN & Inception V3 & 600 & 20 & 26,6 & 41,9 & 21 & 4,2 & 28,3 & 43,6 & 2627 & 1014 & 21 & 613 & 11 & 130 & 13 & 4429 & 1 \\
\hline 49 & Faster RCNN & Inception V3 & 600 & 50 & 28,6 & 44 & 23,5 & 4,9 & 29,9 & 47,9 & 2627 & 1522 & 14 & 1048 & 60 & 153 & 16 & 7437 & 1 \\
\hline 50 & Faster RCNN & Inception V3 & 600 & 100 & 29,2 & 44,6 & 24,3 & 5,4 & 30,2 & 49,4 & 2627 & 2293 & 81 & 1482 & 5 & 206 & 13 & 12,451 & 1 \\
\hline 51 & Faster RCNN & Inception Resnet V2 & 300 & 300 & 28,4 & 47 & 20,5 & 3,6 & 29,3 & 47,1 & 6002 & 14,769 & 103 & 7118 & 104 & 625 & 15 & 63,998 & $0,652,455,535$ \\
\hline 52 & Faster RCNN & Inception Resnet V2 & 300 & 10 & 24,7 & 42,7 & 15,6 & 2,8 & 26,6 & 38,8 & 6002 & 903 & 14 & 518 & 14 & 130 & 14 & 3345 & 1 \\
\hline
\end{tabular}




\begin{tabular}{|c|c|c|c|c|c|c|c|c|c|c|c|c|c|c|c|c|c|c|c|}
\hline & Architecture & Extractor & Resolution & $\begin{array}{l}\# \\
\text { Proposals }\end{array}$ & $\mathrm{mAP}$ & $\begin{array}{l}\mathrm{mAP} \\
\text { (large) }\end{array}$ & $\begin{array}{l}\text { mAP } \\
\text { (medium) }\end{array}$ & $\begin{array}{l}\mathrm{mAP} \\
\text { (small) }\end{array}$ & $\begin{array}{l}\text { mAP @. } \\
75 I O U\end{array}$ & $\begin{array}{l}\text { mAP @. } \\
50 I O U\end{array}$ & $\begin{array}{l}\text { \# model } \\
\text { params }\end{array}$ & Memory & $\begin{array}{l}\text { Memory } \\
\text { std }\end{array}$ & $\mathrm{CPU}$ & $\begin{array}{l}\text { CPU } \\
\text { std }\end{array}$ & GPU & $\begin{array}{l}\text { GPU } \\
\text { std }\end{array}$ & FLOPS & DEA result \\
\hline 53 & Faster RCNN & Inception Resnet V2 & 300 & 20 & 26,6 & 45 & 18,1 & 3,4 & 28,1 & 43 & 6002 & 1295 & 36 & 756 & 19 & 147 & 13 & 5436 & $0,932,407,582$ \\
\hline 54 & Faster RCNN & Inception Resnet V2 & 300 & 50 & 27,9 & 46,3 & 19,9 & 3,7 & 29 & 45,9 & 6002 & 2622 & 69 & 1467 & 23 & 197 & 13 & 11,711 & $0,820,250,506$ \\
\hline 55 & Faster RCNN & Inception Resnet V2 & 300 & 100 & 28,2 & 46,7 & 20,3 & 3,7 & 28,2 & 46,7 & 6002 & 4908 & 212 & 2670 & 16 & 283 & 13 & 22,168 & $0,842,905,455$ \\
\hline 56 & Faster RCNN & Inception Resnet V2 & 600 & 300 & 35,4 & 52,8 & 31,1 & 8,3 & 37,5 & 56,3 & 6002 & 15,799 & 431 & 7652 & 31 & 671 & 13 & 68,043 & 1 \\
\hline 57 & Faster RCNN & Inception Resnet V2 & 600 & 10 & 29 & 46,1 & 22,9 & 5,4 & 31,8 & 43,5 & 6002 & 2173 & 57 & 1138 & 28 & 157 & 14 & 73,9 & $0,947,403,184$ \\
\hline 58 & Faster RCNN & Inception Resnet V2 & 600 & 20 & 32,2 & 49,6 & 27,1 & 6,7 & 34,9 & 49,6 & 6002 & 2651 & 50 & 1297 & 33 & 177 & 14 & 9481 & $0,971,769,717$ \\
\hline 59 & Faster RCNN & Inception Resnet V2 & 600 & 50 & 34,2 & 51,5 & 29,7 & 7,8 & 36,6 & 54,1 & 6002 & 4172 & 119 & 2058 & 62 & 232 & 13 & 15,756 & $0,938,547,812$ \\
\hline 60 & Faster RCNN & Inception Resnet V2 & 600 & 100 & 34,9 & 52,4 & 30,5 & 8,1 & 37,1 & 55,4 & 6002 & 6222 & 257 & 3390 & 47 & 319 & 14 & 26,213 & $0,916,369,468$ \\
\hline 61 & Faster RCNN & Inception Resnet V2 & 300 & 300 & 29,8 & 48,4 & 22,8 & 4,5 & 30,9 & 49,2 & 6002 & 6053 & 267 & 2905 & 66 & 272 & 19 & 66,545 & $0,680,231,761$ \\
\hline 62 & Faster RCNN & Inception Resnet V2 & 300 & 10 & 25,1 & 43,5 & 16,2 & 2,7 & 27,2 & 39 & 6002 & 6207 & 79 & 2937 & 31 & 287 & 15 & 5892 & $0,690,971,126$ \\
\hline 63 & Faster RCNN & Inception Resnet V2 & 300 & 20 & 27,4 & 46,2 & 19,3 & 3,8 & 29,4 & 43,7 & 6002 & 6138 & 109 & 2917 & 24 & 286 & 16 & 7984 & $0,781,504,982$ \\
\hline 64 & Faster RCNN & Inception Resnet V2 & 300 & 50 & 28,9 & 47,7 & 21,3 & 4,4 & 30,4 & 47,1 & 6002 & 5920 & 177 & 3036 & 15 & 283 & 16 & 14,258 & $0,838,422,855$ \\
\hline 65 & Faster RCNN & Inception Resnet V2 & 300 & 100 & 29,5 & 48,1 & 22,1 & 4,5 & 30,7 & 48,5 & 6002 & 6141 & 81 & 2945 & 52 & 281 & 17 & 24,715 & $0,673,962,641$ \\
\hline 66 & Faster RCNN & Inception Resnet V2 & 600 & 300 & 35,7 & 52,5 & 32 & 8,9 & 38 & 56,5 & 6002 & 19,467 & 284 & 10,018 & 491 & 855 & 14 & 78,899 & $0,912,968,467$ \\
\hline 67 & Faster RCNN & Inception Resnet V2 & 600 & 10 & 29,1 & 46,5 & 23,6 & 4,9 & 31,9 & 43,3 & 6002 & 6008 & 111 & 2889 & 34 & 285 & 15 & 18,245 & $0,696,804,143$ \\
\hline 68 & Faster RCNN & Inception Resnet V2 & 600 & 20 & 32,2 & 49,8 & 27,5 & 6,6 & 34,9 & 49,2 & 6002 & 6536 & 186 & 3230 & 92 & 308 & 13 & 20,337 & $0,794,358,534$ \\
\hline 69 & Faster RCNN & Inception Resnet V2 & 600 & 50 & 34,4 & 51,6 & 30,4 & 7,9 & 36,9 & 53,8 & 6002 & 7854 & 19 & 3849 & 29 & 367 & 15 & 26,611 & 1 \\
\hline 70 & Faster RCNN & Inception Resnet V2 & 600 & 100 & 35,3 & 52,1 & 31,5 & 8,5 & 37,6 & 55,6 & 6002 & 10,341 & 296 & 5132 & 38 & 465 & 14 & 37,069 & $0,960,959,275$ \\
\hline 71 & Faster RCNN & MobileNet & 300 & 300 & 16,4 & 27,8 & 9,9 & 1 & 15,5 & 31,5 & 6,06 & 1147 & 28 & 427 & 22 & 94 & 17 & 2523 & $0,889,689,454$ \\
\hline 72 & Faster RCNN & MobileNet & 300 & 10 & 14,4 & 25,3 & 7,3 & 0,8 & 14,2 & 26,5 & 6,06 & 143 & 5 & 100 & 5 & 54 & 7 & 2,55 & 1 \\
\hline 73 & Faster RCNN & MobileNet & 300 & 20 & 15,5 & 27 & 8,6 & 0,9 & 15 & 29,3 & 6,06 & 172 & 6 & 123 & 4 & 61 & 9 & 3,33 & 1 \\
\hline 74 & Faster RCNN & MobileNet & 300 & 50 & 16,2 & 27,7 & 9,5 & 1 & 15,3 & 31 & 6,06 & 284 & 4 & 146 & 4 & 72 & 14 & 5,67 & 1 \\
\hline 75 & Faster RCNN & MobileNet & 300 & 100 & 16,4 & 27,9 & 9,9 & 0,9 & 15,5 & 31,5 & 6,06 & 447 & 17 & 203 & 9 & 81 & 14 & 9,59 & $087,861,171$ \\
\hline 76 & Faster RCNN & MobileNet & 600 & 300 & 19,8 & 29,3 & 17,5 & 3,8 & 18,3 & 38,5 & 6,06 & 1412 & 68 & 610 & 8 & 121 & 16 & 30,5 & 1 \\
\hline 77 & Faster RCNN & MobileNet & 600 & 10 & 15,1 & 23,7 & 11,8 & 2,4 & 14,8 & 28 & 6,06 & 487 & 7 & 273 & 12 & 63 & 6 & 7,82 & 1 \\
\hline 78 & Faster RCNN & MobileNet & 600 & 20 & 17,4 & 26,8 & 14,4 & 2,8 & 16,4 & 33,2 & 6,06 & 507 & 4 & 292 & 2 & 69 & 9 & 8,6 & 1 \\
\hline 79 & Faster RCNN & MobileNet & 600 & 50 & 19,1 & 28,6 & 16,7 & 3,5 & 17,8 & 37 & 6,06 & 572 & 25 & 349 & 11 & 79 & 14 & 1094 & 1 \\
\hline 80 & Faster RCNN & MobileNet & 600 & 100 & 19,7 & 29,2 & 17,4 & 3,7 & 18,1 & 38,3 & 6,06 & 768 & 10 & 376 & 18 & 98 & 14 & 1485 & 1 \\
\hline 81 & R-FCN & Resnet 101 & 300 & 300 & 25,2 & 41,1 & 18,7 & 2,5 & 25,9 & 43,4 & 6848 & 992 & 30 & 444 & 15 & 103 & 6 & 2714 & 1 \\
\hline 82 & R-FCN & Resnet 101 & 300 & 100 & 25,2 & 41,2 & 18,6 & 2,6 & 26 & 43,5 & 6848 & 861 & 47 & 437 & 25 & 94 & 5 & 2714 & 1 \\
\hline 83 & R-FCN & Resnet 101 & 300 & 50 & 24,9 & 40,9 & 1821 & 2,5 & 25,8 & 42,7 & 6848 & 798 & 51 & 425 & 20 & 92 & 6 & 2714 & 1 \\
\hline 84 & R-FCN & Resnet 101 & 300 & 20 & 23,8 & 39,7 & 16,8 & 2,1 & 25,2 & 40,1 & 6848 & 713 & 79 & 413 & 10 & 90 & 6 & 2714 & 1 \\
\hline 85 & R-FCN & Resnet 101 & 300 & 10 & 22,1 & 37,6 & 14,4 & 1,8 & 23,9 & 36,3 & 6848 & 708 & 25 & 428 & 31 & 89 & 6 & 2714 & 1 \\
\hline 86 & R-FCN & Resnet 101 & 600 & 300 & 30,4 & 43,3 & 28,3 & 7,2 & 32 & 51,4 & 6848 & 2878 & 75 & 1536 & 42 & 161 & 8 & 1073 & 1 \\
\hline 87 & R-FCN & Resnet 101 & 600 & 100 & 30,5 & 43,4 & 28,5 & 7,4 & 32,1 & 51,6 & 6848 & 2651 & 19 & 1465 & 62 & 141 & 9 & 1073 & 1 \\
\hline 88 & R-FCN & Resnet 101 & 600 & 50 & 30,1 & 43,3 & 27,9 & 7,3 & 31,8 & 50,4 & 6848 & 2591 & 100 & 1419 & 40 & 134 & 8 & 1073 & 1 \\
\hline 89 & R-FCN & Resnet 101 & 600 & 20 & 28,3 & 41,3 & 25,7 & 6,2 & 30,4 & 46,5 & 6848 & 2600 & 69 & 1503 & 103 & 131 & 8 & 1073 & 1 \\
\hline 90 & R-FCN & Resnet 101 & 600 & 10 & 25,3 & 37,9 & 21,9 & 4,9 & 27,7 & 40,5 & 6848 & 2530 & 34 & 1438 & 64 & 131 & 8 & 1073 & 1 \\
\hline 91 & R-FCN & Resnet 101 & 300 & 300 & 27,6 & 43,3 & 22,7 & 4,4 & 29 & 46,1 & 6848 & 2190 & 26 & 1330 & 25 & 147 & 7 & 10,067 & 1 \\
\hline 92 & R-FCN & Resnet 101 & 300 & 100 & 27,4 & 43,5 & 22,3 & 4,2 & 28,9 & 45,7 & 6848 & 2101 & 97 & 1314 & 21 & 133 & 6 & 10,067 & $0,976,361,082$ \\
\hline 93 & R-FCN & Resnet 101 & 300 & 50 & 27 & 43,3 & 21,6 & 3,7 & 28,7 & 44,5 & 6848 & 2072 & 86 & 1325 & 57 & 128 & 6 & 10,067 & $0,955,884,039$ \\
\hline 94 & R-FCN & Resnet 101 & 300 & 20 & 25,2 & 42 & 18,6 & 3 & 27,4 & 40,7 & 6848 & 2088 & 86 & 1303 & 23 & 127 & 6 & 10,067 & $0,934,326,435$ \\
\hline 95 & R-FCN & Resnet 101 & 300 & 10 & 23,1 & 39,4 & 15,7 & 2,4 & 25,5 & 36,5 & 6848 & 1943 & 93 & 1326 & 57 & 126 & 6 & 10,067 & $0,897,568,909$ \\
\hline 96 & R-FCN & Resnet 101 & 600 & 300 & 31,9 & 43 & 30,8 & 10,4 & 33,7 & 52,4 & 6848 & 7746 & 394 & 4823 & 145 & 389 & 14 & 39,178 & 1 \\
\hline 97 & R-FCN & Resnet 101 & 600 & 100 & 31,7 & 43,2 & 30,7 & 10 & 33,8 & 52,2 & 6848 & 7143 & 97 & 4747 & 125 & 327 & 13 & 39,178 & 1 \\
\hline 98 & R-FCN & Resnet 101 & 600 & 50 & 31,1 & 42,9 & 30,1 & 9,3 & 33,3 & 50,8 & 6848 & 7842 & 163 & 4769 & 108 & 313 & 12 & 39,178 & $0,971,004,451$ \\
\hline 99 & R-FCN & Resnet 101 & 600 & 20 & 28,8 & 41,7 & 26,9 & 7,2 & 31,5 & 46,1 & 6848 & 7562 & 251 & 4801 & 134 & 304 & 13 & 39,178 & $0,716,476,904$ \\
\hline 100 & R-FCN & Resnet 101 & 600 & 10 & 25,6 & 38,8 & 22,6 & 5,4 & 28,3 & 40,1 & 6848 & 7553 & 244 & 4685 & 113 & 302 & 13 & 39,178 & $061,047,968$ \\
\hline 101 & R-FCN & Inception V2 & 300 & 300 & 15,4 & 27,8 & 7 & 0 & 14,5 & 29,5 & 1806 & 286 & 14 & 144 & 7 & 72 & 5 & 5,09 & 1 \\
\hline 102 & R-FCN & Inception V2 & 300 & 100 & 15,5 & 27,9 & 7,2 & 1 & 14,6 & 29,9 & 1806 & 208 & 11 & 125 & 6 & 69 & 6 & 5,09 & 1 \\
\hline 103 & R-FCN & Inception V2 & 300 & 50 & 15,3 & 27,7 & 7,1 & 1 & 14,5 & 29,6 & 1806 & 202 & 13 & 125 & 6 & 66 & 6 & 5,09 & 1 \\
\hline
\end{tabular}




\begin{tabular}{|c|c|c|c|c|c|c|c|c|c|c|c|c|c|c|c|c|c|c|c|}
\hline & Architecture & Extractor & Resolution & $\begin{array}{l}\# \\
\text { Proposals }\end{array}$ & $\mathrm{mAP}$ & $\begin{array}{l}\text { mAP } \\
\text { (large) }\end{array}$ & $\begin{array}{l}\mathrm{mAP} \\
\text { (medium) }\end{array}$ & $\begin{array}{l}\mathrm{mAP} \\
\text { (small) }\end{array}$ & $\begin{array}{l}\text { mAP @. } \\
75 I O U\end{array}$ & $\begin{array}{l}\text { mAP @. } \\
50 I O U\end{array}$ & $\begin{array}{l}\text { \# model } \\
\text { params }\end{array}$ & Memory & $\begin{array}{l}\text { Memory } \\
\text { std }\end{array}$ & CPU & $\begin{array}{l}\text { CPU } \\
\text { std }\end{array}$ & GPU & $\begin{array}{l}\text { GPU } \\
\text { std }\end{array}$ & FLOPS & DEA result \\
\hline 104 & R-FCN & Inception V2 & 300 & 20 & 14,7 & 26,8 & 6,5 & 0 & 14,1 & 27,9 & 1806 & 177 & 10 & 123 & 8 & 64 & 6 & 5,09 & 1 \\
\hline 105 & R-FCN & Inception V2 & 300 & 10 & 13,8 & 25,2 & 5,7 & 0 & 13,6 & 25,6 & 1806 & 164 & 12 & 121 & 7 & 66 & 6 & 5,09 & 1 \\
\hline 106 & R-FCN & Inception V2 & 600 & 300 & 19,8 & 31,8 & 15,2 & 1,8 & 18,2 & 38,3 & 1806 & 715 & 6 & 445 & 16 & 101 & 6 & 1985 & 1 \\
\hline 107 & R-FCN & Inception V2 & 600 & 100 & 20,1 & 31,9 & 15,6 & 2 & 18,5 & 39,1 & 1806 & 647 & 19 & 394 & 15 & 87 & 6 & 1985 & 1 \\
\hline 108 & R-FCN & Inception V2 & 600 & 50 & 19,7 & 31,6 & 15 & 2 & 18,3 & 38,1 & 1806 & 624 & 25 & 391 & 19 & 84 & 7 & 1985 & 1 \\
\hline 109 & R-FCN & Inception V2 & 600 & 20 & 18,3 & 30,1 & 13,2 & 1,8 & 17,4 & 34,7 & 1806 & 609 & 9 & 378 & 5 & 81 & 6 & 1985 & 1 \\
\hline 110 & R-FCN & Inception V2 & 600 & 10 & 15,9 & 26,5 & 10,6 & 1,6 & 15,6 & 29,5 & 1806 & 581 & 12 & 376 & 8 & 79 & 7 & 1985 & $0,994,897,448$ \\
\hline 111 & R-FCN & Inception Resnet V2 & 300 & 300 & 22,5 & 39,6 & 13,2 & 1 & 22,7 & 39,8 & 6506 & 696 & 108 & 339 & 10 & 108 & 6 & 1499 & 1 \\
\hline 112 & R-FCN & Inception Resnet V2 & 300 & 100 & 22,8 & 39,8 & 13,8 & 1,1 & 23 & 40,7 & 6506 & 642 & 71 & 315 & 13 & 100 & 7 & 1499 & 1 \\
\hline 113 & R-FCN & Inception Resnet V2 & 300 & 50 & 22,8 & 39,6 & 14 & 1,2 & 23 & 40,6 & 6506 & 679 & 33 & 314 & 7 & 101 & 6 & 1499 & 1 \\
\hline 114 & R-FCN & Inception Resnet V2 & 300 & 20 & 22,3 & 38,9 & 13,5 & 1 & 22,8 & 39,1 & 6506 & 611 & 42 & 312 & 13 & 99 & 6 & 1499 & 1 \\
\hline 115 & R-FCN & Inception Resnet V2 & 300 & 10 & 21,1 & 37,5 & 12,1 & 1,1 & 22,1 & 36 & 6506 & 637 & 96 & 324 & 10 & 98 & 7 & 1499 & 1 \\
\hline 116 & R-FCN & Inception Resnet V2 & 600 & 300 & 30,7 & 46,2 & 26,8 & 5,6 & 31,7 & 52,3 & 6506 & 2173 & 108 & 1048 & 24 & 165 & 6 & 6396 & 1 \\
\hline 117 & R-FCN & Inception Resnet V2 & 600 & 100 & 30,9 & 46,4 & 27 & 6,1 & 31,9 & 52,8 & 6506 & 2121 & 36 & 993 & 26 & 150 & 6 & 6396 & 1 \\
\hline 118 & $\mathrm{R}-\mathrm{FCN}$ & Inception Resnet V2 & 600 & 50 & 30,7 & 46,1 & 26,8 & 6,2 & 32 & 52,3 & 6506 & 2150 & 83 & 1060 & 41 & 146 & 6 & 6396 & 1 \\
\hline 119 & R-FCN & Inception Resnet V2 & 600 & 20 & 29,3 & 44,9 & 25 & 5,4 & 31,1 & 48,8 & 6506 & 2051 & 12 & 1007 & 30 & 144 & 6 & 6396 & 1 \\
\hline 120 & R-FCN & Inception Resnet V2 & 600 & 10 & 27,1 & 42,7 & 22,1 & 4,4 & 29,3 & 43,9 & 6506 & 2151 & 62 & 1034 & 12 & 141 & 5 & 6396 & 1 \\
\hline 121 & $\mathrm{R}-\mathrm{FCN}$ & Inception Resnet V2 & 300 & 300 & 26 & 43,5 & 18,7 & 2 & 26,4 & 45,3 & 6506 & 1609 & 37 & 827 & 14 & 142 & 7 & 4774 & 1 \\
\hline 122 & $\mathrm{R}-\mathrm{FCN}$ & Inception Resnet V2 & 300 & 100 & 26 & 43,5 & 18,6 & 2,1 & 26,4 & 45,2 & 6506 & 1557 & 28 & 841 & 10 & 134 & 6 & 4774 & 1 \\
\hline 123 & R-FCN & Inception Resnet V2 & 300 & 50 & 25,6 & 43,2 & 17,9 & 1,9 & 26,1 & 44,3 & 6506 & 1564 & 14 & 808 & 5 & 133 & 7 & 4774 & 1 \\
\hline 124 & R-FCN & Inception Resnet V2 & 300 & 20 & 24,4 & 42,4 & 15,9 & 1,7 & 25,4 & 41,3 & 6506 & 1499 & 83 & 796 & 3 & 128 & 8 & 4774 & 1 \\
\hline 125 & R-FCN & Inception Resnet V2 & 300 & 10 & 22,5 & 40,1 & 13,5 & 1,5 & 23,9 & 37,4 & 6506 & 1501 & 102 & 791 & 14 & 131 & 6 & 4774 & $0,937,080,879$ \\
\hline 126 & R-FCN & Inception Resnet V2 & 600 & 300 & 32,3 & 46,9 & 29,3 & 8 & 33,8 & 53,9 & 6506 & 7289 & 46 & 3220 & 60 & 368 & 8 & 20,545 & 1 \\
\hline 127 & R-FCN & Inception Resnet V2 & 600 & 100 & 32,1 & 46,8 & 29,3 & 8 & 33,7 & 53,7 & 6506 & 6653 & 53 & 3319 & 70 & 328 & 6 & 20,545 & 1 \\
\hline 128 & R-FCN & Inception Resnet V2 & 600 & 50 & 31,5 & 46,4 & 28,6 & 7,6 & 33,3 & 52,2 & 6506 & 6771 & 258 & 3315 & 32 & 313 & 7 & 20,545 & 1 \\
\hline 129 & R-FCN & Inception Resnet V2 & 600 & 20 & 29,7 & 45,2 & 26,4 & 6,7 & 32 & 48,3 & 6506 & 6958 & 137 & 3318 & 100 & 302 & 7 & 20,545 & $0,898,411,248$ \\
\hline 130 & R-FCN & Inception Resnet V2 & 600 & 10 & 27,1 & 42,7 & 22,7 & 4,8 & 29,7 & 42,9 & 6506 & 7001 & 40 & 3320 & 21 & 299 & 7 & 20,545 & $0,941,140,671$ \\
\hline 131 & R-FCN & MobileNet & 300 & 300 & 15 & 26,3 & 7,6 & 0 & 13,7 & 29,4 & 10,8 & 246 & 4 & 114 & 4 & 66 & 5 & 2,44 & 1 \\
\hline 132 & R-FCN & MobileNet & 300 & 100 & 15,2 & 26,5 & 7,9 & 1 & 13,8 & 30 & 10,8 & 170 & 10 & 94 & 8 & 57 & 7 & 2,44 & 1 \\
\hline 133 & R-FCN & MobileNet & 300 & 50 & 15,2 & 26,5 & 7,8 & 1 & 13,8 & 30 & 10,8 & 159 & 10 & 92 & 8 & 60 & 5 & 2,44 & 1 \\
\hline 134 & $\mathrm{R}-\mathrm{FCN}$ & MobileNet & 300 & 20 & 14,7 & 25,9 & 7,3 & 1 & 13,6 & 28,7 & 10,8 & 150 & 10 & 96 & 7 & 59 & 5 & 2,44 & 1 \\
\hline 135 & R-FCN & MobileNet & 300 & 10 & 13,8 & 24,4 & 6,5 & 1 & 13,1 & 26,4 & 10,8 & 137 & 11 & 97 & 5 & 58 & 5 & 2,44 & 1 \\
\hline 136 & R-FCN & MobileNet & 600 & 300 & 16,5 & 25,8 & 13,2 & 1,5 & 14 & 33,8 & 10,8 & 607 & 17 & 341 & 25 & 86 & 5 & 9,49 & 1 \\
\hline 137 & R-FCN & MobileNet & 600 & 100 & 17 & 26,2 & 13,8 & 1,8 & 14,3 & 35,1 & 10,8 & 537 & 6 & 296 & 8 & 73 & 6 & 9,49 & 1 \\
\hline 138 & R-FCN & MobileNet & 600 & 50 & 16,9 & 26 & 13,7 & 2 & 14,2 & 34,8 & 10,8 & 514 & 2 & 283 & 8 & 70 & 6 & 9,49 & 1 \\
\hline 139 & R-FCN & MobileNet & 600 & 20 & 15,9 & 24,9 & 12,6 & 1,8 & 13,7 & 32,3 & 10,8 & 511 & 15 & 285 & 8 & 69 & 5 & 9,49 & 1 \\
\hline 140 & R-FCN & MobileNet & 600 & 10 & 14,1 & 22,2 & 10,6 & 1,3 & 12,5 & 27,9 & 10,8 & 507 & 20 & 280 & 9 & 67 & 5 & 9,49 & 1 \\
\hline
\end{tabular}




\begin{tabular}{|c|c|c|c|c|c|c|c|c|c|c|c|c|c|c|c|c|c|c|c|}
\hline & Architecture & Extractor & Resolution & $\begin{array}{l}\# \\
\text { Proposals } \\
\end{array}$ & $\mathrm{mAP}$ & $\begin{array}{l}\text { mAP } \\
\text { (large) }\end{array}$ & $\begin{array}{l}\text { mAP } \\
\text { (medium) }\end{array}$ & $\begin{array}{l}\text { mAP } \\
\text { (small) }\end{array}$ & $\begin{array}{l}\text { mAP @. } \\
75 I O U\end{array}$ & $\begin{array}{l}\text { mAP @. } \\
50 I O U\end{array}$ & $\begin{array}{l}\text { \# model } \\
\text { params }\end{array}$ & Memory & $\begin{array}{l}\text { Memory } \\
\text { std }\end{array}$ & $\mathrm{CPU}$ & $\begin{array}{l}\mathrm{CPU} \\
\text { std }\end{array}$ & GPU & $\begin{array}{l}\text { GPU } \\
\text { std }\end{array}$ & FLOPS & DEA result \\
\hline 1 & Faster RCNN & VGG & 300 & 300 & 22,9 & 37,7 & 16,4 & 2,4 & 22,9 & 41,1 & 13,851 & 1699 & 81 & 1286 & 27 & 201 & 16 & 6432 & $099,989,176$ \\
\hline 2 & Faster RCNN & VGG & 300 & 10 & 19,6 & 32,8 & 12,7 & 1,7 & 20,4 & 33,4 & 13,851 & 1703 & 30 & 1322 & 41 & 203 & 16 & 2912 & $0,999,729,094$ \\
\hline 3 & Faster RCNN & VGG & 300 & 20 & 21,5 & 35,7 & 14,8 & 2,1 & 21,9 & 37,6 & 13,851 & 1870 & 183 & 1261 & 5 & 204 & 15 & 3033 & $0,993,706,578$ \\
\hline 4 & Faster RCNN & VGG & 300 & 50 & 22,5 & 37 & 16,1 & 2,3 & 22,5 & 40,2 & 13,851 & 1842 & 258 & 1280 & 41 & 202 & 15 & 3397 & $0,973,411,705$ \\
\hline 5 & Faster RCNN & VGG & 300 & 100 & 22,9 & 37,5 & 16,4 & 2,5 & 22,8 & 41 & 13,851 & 1713 & 152 & 1272 & 8 & 201 & 17 & 4004 & 0,997,057,668 \\
\hline 6 & Faster RCNN & VGG & 600 & 300 & 25,7 & 37,2 & 23 & 5,6 & 25,7 & 46 & 13,851 & 2048 & 210 & 1668 & 71 & 247 & 14 & 14,959 & $0,971,625,067$ \\
\hline 7 & Faster RCNN & VGG & 600 & 10 & 19 & 28,3 & 15,6 & 3,7 & 20,1 & 32,2 & 13,851 & 1853 & 388 & 1272 & 7 & 206 & 15 & 11,439 & $0,970,515,927$ \\
\hline 8 & Faster RCNN & VGG & 600 & 20 & 22,3 & 32,8 & 19,3 & 5 & 23,1 & 38,7 & 13,851 & 1984 & 182 & 1282 & 5 & 205 & 16 & 1156 & $0,987,513,333$ \\
\hline 9 & Faster RCNN & VGG & 600 & 50 & 24,8 & 35,6 & 22,2 & 5,9 & 25,3 & 44 & 13,851 & 1822 & 29 & 1327 & 6 & 209 & 16 & 11,925 & 1 \\
\hline 10 & Faster RCNN & VGG & 600 & 100 & 25,6 & 36,5 & 22,9 & 6,2 & 25,8 & 45,6 & 13,851 & 1948 & 210 & 1384 & 11 & 217 & 15 & 12,532 & $099,395,933$ \\
\hline 11 & Faster RCNN & Resnet 101 & 300 & 300 & 26,5 & 43,5 & 19 & 3 & 27,2 & 44,6 & 6315 & 3236 & 190 & 2484 & 28 & 206 & 14 & 23,942 & $0,713,567,647$ \\
\hline 12 & Faster RCNN & Resnet 101 & 300 & 10 & 23,1 & 39,3 & 14,8 & 2,3 & 24,6 & 37,1 & 6315 & 560 & 19 & 355 & 13 & 92 & 13 & 2682 & 1 \\
\hline 13 & Faster RCNN & Resnet 101 & 300 & 20 & 24,8 & 41,6 & 17,1 & 2,6 & 26,1 & 40,9 & 6315 & 664 & 40 & 427 & 19 & 102 & 15 & 3415 & 0,997,407,914 \\
\hline 14 & Faster RCNN & Resnet 101 & 300 & 50 & 25,8 & 42,7 & 18,4 & 2,9 & 26,7 & 43,4 & 6315 & 1021 & 10 & 634 & 15 & 112 & 17 & 5614 & $0,991,107,729$ \\
\hline 15 & Faster RCNN & Resnet 101 & 300 & 100 & 26,3 & 43,2 & 18,8 & 3,1 & 27 & 44,3 & 6315 & 1459 & 33 & 1029 & 12 & 136 & 14 & 92,8 & 0,914,321,099 \\
\hline 16 & Faster RCNN & Resnet 101 & 600 & 300 & 32,2 & 47,1 & 28,7 & 7,5 & 33,9 & 52,6 & 6315 & 4681 & 209 & 3127 & 17 & 248 & 14 & 29,661 & $0,952,743,925$ \\
\hline 17 & Faster RCNN & Resnet 101 & 600 & 10 & 26,3 & 40 & 21,6 & 4,6 & 29,1 & 40,6 & 6315 & 1884 & 132 & 967 & 6 & 115 & 15 & 8402 & $098,105,357$ \\
\hline 18 & Faster RCNN & Resnet 101 & 600 & 20 & 29,4 & 44,3 & 25,3 & 6,2 & 31,8 & 46,4 & 6315 & 1985 & 81 & 1085 & 93 & 126 & 16 & 9135 & $098,744,646$ \\
\hline 19 & Faster RCNN & Resnet 101 & 600 & 50 & 31,3 & 46,1 & 27,6 & 7,2 & 33,4 & 50,6 & 6315 & 2267 & 106 & 1287 & 46 & 140 & 17 & 11,334 & $0,989,963,525$ \\
\hline 20 & Faster RCNN & Resnet 101 & 600 & 100 & 32 & 46,7 & 28,5 & 7,5 & 33,9 & 52 & 6315 & 2756 & 138 & 1652 & 47 & 164 & 17 & 150 & $0,958,338,596$ \\
\hline 21 & Faster RCNN & Resnet 101 & 300 & 300 & 29,5 & 46,1 & 23,4 & 5,2 & 31,1 & 48,3 & 6315 & 4271 & 262 & 2930 & 23 & 233 & 15 & 28,999 & $0,801,307,984$ \\
\hline 22 & Faster RCNN & Resnet 101 & 300 & 10 & 24,5 & 41,1 & 16,6 & 3,5 & 27 & 37,9 & 6315 & 1378 & 44 & 789 & 23 & 108 & 13 & 7739 & $0,945,030,885$ \\
\hline 23 & Faster RCNN & Resnet 101 & 300 & 20 & 26,9 & 43,9 & 19,9 & 4,3 & 29,1 & 42,7 & 6315 & 1464 & 49 & 875 & 10 & 120 & 16 & 8472 & $0,975,020,567$ \\
\hline 24 & Faster RCNN & Resnet 101 & 300 & 50 & 28,7 & 45,4 & 22,3 & 4,9 & 30,6 & 46,5 & 6315 & 1703 & 42 & 1098 & 8 & 132 & 18 & 10,671 & $0,984,282,946$ \\
\hline 25 & Faster RCNN & Resnet 101 & 300 & 100 & 29,2 & 45,8 & 23,1 & 5,2 & 30,9 & 47,8 & 6315 & 2218 & 162 & 1502 & 12 & 161 & 15 & 14,337 & $0,916,242,977$ \\
\hline 26 & Faster RCNN & Resnet 101 & 600 & 300 & 34,4 & 47,7 & 31,8 & 10,7 & 37,1 & 54,6 & 6315 & 8043 & 301 & 4859 & 37 & 390 & 15 & 49,247 & $0,940,374,452$ \\
\hline 27 & Faster RCNN & Resnet 101 & 600 & 10 & 26,7 & 40,7 & 22,2 & 5,1 & 29,6 & 40,2 & 6315 & 5056 & 35 & 2672 & 34 & 196 & 16 & 27,987 & $0,800,751,143$ \\
\hline 28 & Faster RCNN & Resnet 101 & 600 & 20 & 30 & 44,6 & 26,8 & 6,9 & 33 & 46,2 & 6315 & 5146 & 143 & 2729 & 44 & 214 & 15 & 28,721 & $0,810,501,842$ \\
\hline 29 & Faster RCNN & Resnet 101 & 600 & 50 & 32,7 & 46,6 & 29,9 & 8,9 & 35,5 & 51,2 & 6315 & 5375 & 79 & 3047 & 113 & 234 & 16 & 3092 & $088,662,145$ \\
\hline 30 & Faster RCNN & Resnet 101 & 600 & 100 & 33,7 & 47,4 & 31,1 & 9,9 & 36,5 & 53,4 & 6315 & 5518 & 135 & 3384 & 65 & 267 & 16 & 34,585 & $0,917,106,319$ \\
\hline 31 & Faster RCNN & Inception V2 & 300 & 300 & 15,4 & 27,4 & 7,7 & 0,5 & 14,8 & 29 & 1331 & 2370 & 51 & 1388 & 5 & 178 & 15 & 11,822 & $0,587,621,848$ \\
\hline 32 & Faster RCNN & Inception V2 & 300 & 10 & 13,3 & 24,3 & 5,2 & 0,3 & 13,4 & 23,7 & 1331 & 211 & 6 & 153 & 7 & 79 & 13 & 7,63 & $0,935,649,345$ \\
\hline 33 & Faster RCNN & Inception V2 & 300 & 20 & 14,3 & 26 & 6,3 & 0,3 & 14,1 & 26,3 & 1331 & 280 & 11 & 198 & 6 & 90 & 13 & 1144 & $0,847,857,988$ \\
\hline 34 & Faster RCNN & Inception V2 & 300 & 50 & 15 & 26,9 & 7,2 & 0,5 & 14,5 & 28 & 1331 & 495 & 21 & 327 & 7 & 99 & 17 & 2288 & 0,719,987,109 \\
\hline 35 & Faster RCNN & Inception V2 & 300 & 100 & 15,3 & 27,3 & 7,4 & 0,5 & 14,7 & 28,5 & 1331 & 861 & 51 & 535 & 7 & 118 & 15 & 4195 & 0,616,208,002 \\
\hline 36 & Faster RCNN & Inception V2 & 600 & 300 & 21,9 & 34,4 & 17,6 & 3,1 & 21,8 & 39,5 & 1331 & 2596 & 63 & 1611 & 21 & 206 & 15 & 12,957 & $0,682,417,576$ \\
\hline 37 & Faster RCNN & Inception V2 & 600 & 10 & 16,4 & 27,3 & 10,7 & 1,9 & 17,2 & 28 & 1331 & 572 & 11 & 383 & 7 & 82 & 12 & 1898 & $0,909,673,555$ \\
\hline 38 & Faster RCNN & Inception V2 & 600 & 20 & 19 & 31,4 & 13,6 & 2,2 & 19,6 & 33,3 & 1331 & 669 & 16 & 446 & 22 & 98 & 13 & 2279 & $0,897,560,228$ \\
\hline 39 & Faster RCNN & Inception V2 & 600 & 50 & 20,8 & 33,5 & 16,1 & 2,5 & 21 & 37,2 & 1331 & 871 & 17 & 574 & 22 & 116 & 15 & 3423 & $0,879,550,227$ \\
\hline 40 & Faster RCNN & Inception V2 & 600 & 100 & 21,5 & 34 & 16,9 & 2,8 & 21,5 & 38,7 & 1331 & 1152 & 42 & 769 & 16 & 104 & 4 & 53,3 & $0,901,831,205$ \\
\hline 41 & Faster RCNN & Inception V3 & 300 & 300 & 23,6 & 40,6 & 15,1 & 1,6 & 23,5 & 41,4 & 2627 & 5016 & 128 & 3214 & 18 & 353 & 14 & 3066 & $044,984,218$ \\
\hline 42 & Faster RCNN & Inception V3 & 300 & 10 & 20,2 & 36,1 & 11,1 & 1,3 & 21,3 & 33,7 & 2627 & 337 & 5 & 252 & 9 & 101 & 13 & 1577 & $0,996,977,915$ \\
\hline 43 & Faster RCNN & Inception V3 & 300 & 20 & 21,9 & 38,4 & 12,9 & 1,6 & 22,6 & 37,3 & 2627 & 487 & 13 & 395 & 30 & 109 & 13 & 25,8 & 0,959,931,055 \\
\hline 44 & Faster RCNN & Inception V3 & 300 & 50 & 23 & 39,8 & 14,4 & 1,7 & 23,2 & 40,1 & 2627 & 1017 & 40 & 669 & 11 & 137 & 13 & 5589 & $0,809,622,795$ \\
\hline 45 & Faster RCNN & Inception V3 & 300 & 100 & 23,4 & 40,3 & 14,9 & 1,7 & 23,4 & 41 & 2627 & 1734 & 106 & 1188 & 22 & 181 & 14 & 10,603 & $0,638,689,263$ \\
\hline 46 & Faster RCNN & Inception V3 & 600 & 300 & 29,6 & 44,9 & 25 & 5,3 & 30,6 & 50,2 & 2627 & 5386 & 143 & 3662 & 153 & 385 & 13 & 32,509 & $0,528,034,572$ \\
\hline 47 & Faster RCNN & Inception V3 & 600 & 10 & 23,9 & 38,5 & 17,4 & 3,2 & 25,9 & 38,1 & 2627 & 799 & 71 & 525 & 18 & 116 & 12 & 3426 & $0,960,555,055$ \\
\hline 48 & Faster RCNN & Inception V3 & 600 & 20 & 26,6 & 41,9 & 21 & 4,2 & 28,3 & 43,6 & 2627 & 1014 & 21 & 613 & 11 & 130 & 13 & 4429 & 0,994,017,092 \\
\hline 49 & Faster RCNN & Inception V3 & 600 & 50 & 28,6 & 44 & 23,5 & 4,9 & 29,9 & 47,9 & 2627 & 1522 & 14 & 1048 & 60 & 153 & 16 & 7437 & $0,967,415,353$ \\
\hline 50 & Faster RCNN & Inception V3 & 600 & 100 & 29,2 & 44,6 & 24,3 & 5,4 & 30,2 & 49,4 & 2627 & 2293 & 81 & 1482 & 5 & 206 & 13 & 12,451 & $090,540,433$ \\
\hline 51 & Faster RCNN & Inception Resnet V2 & 300 & 300 & 28,4 & 47 & 20,5 & 3,6 & 29,3 & 47,1 & 6002 & 14,769 & 103 & 7118 & 104 & 625 & 15 & 63,998 & $0,353,609,455$ \\
\hline 52 & Faster RCNN & Inception Resnet V2 & 300 & 10 & 24,7 & 42,7 & 15,6 & 2,8 & 26,6 & 38,8 & 6002 & 903 & 14 & 518 & 14 & 130 & 14 & 3345 & $0,982,884,168$ \\
\hline
\end{tabular}




\begin{tabular}{|c|c|c|c|c|c|c|c|c|c|c|c|c|c|c|c|c|c|c|c|}
\hline & Architecture & Extractor & Resolution & $\begin{array}{l}\# \\
\text { Proposals }\end{array}$ & $\mathrm{mAP}$ & $\begin{array}{l}\mathrm{mAP} \\
\text { (large) }\end{array}$ & $\begin{array}{l}\text { mAP } \\
\text { (medium) }\end{array}$ & $\begin{array}{l}\text { mAP } \\
\text { (small) }\end{array}$ & $\begin{array}{l}\text { mAP @. } \\
75 I O U\end{array}$ & $\begin{array}{l}\text { mAP @. } \\
50 I O U\end{array}$ & $\begin{array}{l}\text { \# model } \\
\text { params }\end{array}$ & Memory & $\begin{array}{l}\text { Memory } \\
\text { std }\end{array}$ & CPU & $\begin{array}{l}\text { CPU } \\
\text { std }\end{array}$ & GPU & $\begin{array}{l}\text { GPU } \\
\text { std }\end{array}$ & FLOPS & DEA result \\
\hline 53 & Faster RCNN & Inception Resnet V2 & 300 & 20 & 26,6 & 45 & 18,1 & 3,4 & 28,1 & 43 & 6002 & 1295 & 36 & 756 & 19 & 147 & 13 & 5436 & $0,910,115,625$ \\
\hline 54 & Faster RCNN & Inception Resnet V2 & 300 & 50 & 27,9 & 46,3 & 19,9 & 3,7 & 29 & 45,9 & 6002 & 2622 & 69 & 1467 & 23 & 197 & 13 & 11,711 & $0,743,147,203$ \\
\hline 55 & Faster RCNN & Inception Resnet V2 & 300 & 100 & 28,2 & 46,7 & 20,3 & 3,7 & 28,2 & 46,7 & 6002 & 4908 & 212 & 2670 & 16 & 283 & 13 & 22,168 & $0,594,708,494$ \\
\hline 56 & Faster RCNN & Inception Resnet V2 & 600 & 300 & 35,4 & 52,8 & 31,1 & 8,3 & 37,5 & 56,3 & 6002 & 15,799 & 431 & 7652 & 31 & 671 & 13 & 68,043 & $0,694,609,731$ \\
\hline 57 & Faster RCNN & Inception Resnet V2 & 600 & 10 & 29 & 46,1 & 22,9 & 5,4 & 31,8 & 43,5 & 6002 & 2173 & 57 & 1138 & 28 & 157 & 14 & 73,9 & $0,934,164,306$ \\
\hline 58 & Faster RCNN & Inception Resnet V2 & 600 & 20 & 32,2 & 49,6 & 27,1 & 6,7 & 34,9 & 49,6 & 6002 & 2651 & 50 & 1297 & 33 & 177 & 14 & 9481 & $0,965,421,866$ \\
\hline 59 & Faster RCNN & Inception Resnet V2 & 600 & 50 & 34,2 & 51,5 & 29,7 & 7,8 & 36,6 & 54,1 & 6002 & 4172 & 119 & 2058 & 62 & 232 & 13 & 15,756 & $0,858,164,779$ \\
\hline 60 & Faster RCNN & Inception Resnet V2 & 600 & 100 & 34,9 & 52,4 & 30,5 & 8,1 & 37,1 & 55,4 & 6002 & 6222 & 257 & 3390 & 47 & 319 & 14 & 26,213 & $0,767,438,361$ \\
\hline 61 & Faster RCNN & Inception Resnet V2 & 300 & 300 & 29,8 & 48,4 & 22,8 & 4,5 & 30,9 & 49,2 & 6002 & 6053 & 267 & 2905 & 66 & 272 & 19 & 66,545 & $0,640,190,174$ \\
\hline 62 & Faster RCNN & Inception Resnet V2 & 300 & 10 & 25,1 & 43,5 & 16,2 & 2,7 & 27,2 & 39 & 6002 & 6207 & 79 & 2937 & 31 & 287 & 15 & 5892 & $0,577,541,319$ \\
\hline 63 & Faster RCNN & Inception Resnet V2 & 300 & 20 & 27,4 & 46,2 & 19,3 & 3,8 & 29,4 & 43,7 & 6002 & 6138 & 109 & 2917 & 24 & 286 & 16 & 7984 & $0,626,636,227$ \\
\hline 64 & Faster RCNN & Inception Resnet V2 & 300 & 50 & 28,9 & 47,7 & 21,3 & 4,4 & 30,4 & 47,1 & 6002 & 5920 & 177 & 3036 & 15 & 283 & 16 & 14,258 & $0,642,692,331$ \\
\hline 65 & Faster RCNN & Inception Resnet V2 & 300 & 100 & 29,5 & 48,1 & 22,1 & 4,5 & 30,7 & 48,5 & 6002 & 6141 & 81 & 2945 & 52 & 281 & 17 & 24,715 & $061,652,699$ \\
\hline 66 & Faster RCNN & Inception Resnet V2 & 600 & 300 & 35,7 & 52,5 & 32 & 8,9 & 38 & 56,5 & 6002 & 19,467 & 284 & 10,018 & 491 & 855 & 14 & 78,899 & $040,432,115$ \\
\hline 67 & Faster RCNN & Inception Resnet V2 & 600 & 10 & 29,1 & 46,5 & 23,6 & 4,9 & 31,9 & 43,3 & 6002 & 6008 & 111 & 2889 & 34 & 285 & 15 & 18,245 & $0,636,675,846$ \\
\hline 68 & Faster RCNN & Inception Resnet V2 & 600 & 20 & 32,2 & 49,8 & 27,5 & 6,6 & 34,9 & 49,2 & 6002 & 6536 & 186 & 3230 & 92 & 308 & 13 & 20,337 & $0,641,461,964$ \\
\hline 69 & Faster RCNN & Inception Resnet V2 & 600 & 50 & 34,4 & 51,6 & 30,4 & 7,9 & 36,9 & 53,8 & 6002 & 7854 & 19 & 3849 & 29 & 367 & 15 & 26,611 & $0,977,820,333$ \\
\hline 70 & Faster RCNN & Inception Resnet V2 & 600 & 100 & 35,3 & 52,1 & 31,5 & 8,5 & 37,6 & 55,6 & 6002 & 10,341 & 296 & 5132 & 38 & 465 & 14 & 37,069 & $0,721,956,602$ \\
\hline 71 & Faster RCNN & MobileNet & 300 & 300 & 16,4 & 27,8 & 9,9 & 1 & 15,5 & 31,5 & 6,06 & 1147 & 28 & 427 & 22 & 94 & 17 & 2523 & $0,893,221,145$ \\
\hline 72 & Faster RCNN & MobileNet & 300 & 10 & 14,4 & 25,3 & 7,3 & 0,8 & 14,2 & 26,5 & 6,06 & 143 & 5 & 100 & 5 & 54 & 7 & 2,55 & 099,989,099 \\
\hline 73 & Faster RCNN & MobileNet & 300 & 20 & 15,5 & 27 & 8,6 & 0,9 & 15 & 29,3 & 6,06 & 172 & 6 & 123 & 4 & 61 & 9 & 3,33 & $0,999,367,307$ \\
\hline 74 & Faster RCNN & MobileNet & 300 & 50 & 16,2 & 27,7 & 9,5 & 1 & 15,3 & 31 & 6,06 & 284 & 4 & 146 & 4 & 72 & 14 & 5,67 & $0,990,888,468$ \\
\hline 75 & Faster RCNN & MobileNet & 300 & 100 & 16,4 & 27,9 & 9,9 & 0,9 & 15,5 & 31,5 & 6,06 & 447 & 17 & 203 & 9 & 81 & 14 & 9,59 & $0,927,673,111$ \\
\hline 76 & Faster RCNN & MobileNet & 600 & 300 & 19,8 & 29,3 & 17,5 & 3,8 & 18,3 & 38,5 & 6,06 & 1412 & 68 & 610 & 8 & 121 & 16 & 30,5 & $0,992,400,523$ \\
\hline 77 & Faster RCNN & MobileNet & 600 & 10 & 15,1 & 23,7 & 11,8 & 2,4 & 14,8 & 28 & 6,06 & 487 & 7 & 273 & 12 & 63 & 6 & 7,82 & $0,983,078,006$ \\
\hline 78 & Faster RCNN & MobileNet & 600 & 20 & 17,4 & 26,8 & 14,4 & 2,8 & 16,4 & 33,2 & 6,06 & 507 & 4 & 292 & 2 & 69 & 9 & 8,6 & $0,999,377,036$ \\
\hline 79 & Faster RCNN & MobileNet & 600 & 50 & 19,1 & 28,6 & 16,7 & 3,5 & 17,8 & 37 & 6,06 & 572 & 25 & 349 & 11 & 79 & 14 & 1094 & $0,999,729,055$ \\
\hline 80 & Faster RCNN & MobileNet & 600 & 100 & 19,7 & 29,2 & 17,4 & 3,7 & 18,1 & 38,3 & 6,06 & 768 & 10 & 376 & 18 & 98 & 14 & 1485 & $0,998,202,182$ \\
\hline 81 & R-FCN & Resnet 101 & 300 & 300 & 25,2 & 41,1 & 18,7 & 2,5 & 25,9 & 43,4 & 6848 & 992 & 30 & 444 & 15 & 103 & 6 & 2714 & 1 \\
\hline 82 & R-FCN & Resnet 101 & 300 & 100 & 25,2 & 41,2 & 18,6 & 2,6 & 26 & 43,5 & 6848 & 861 & 47 & 437 & 25 & 94 & 5 & 2714 & $0,998,793,072$ \\
\hline 83 & R-FCN & Resnet 101 & 300 & 50 & 24,9 & 40,9 & 1821 & 2,5 & 25,8 & 42,7 & 6848 & 798 & 51 & 425 & 20 & 92 & 6 & 2714 & $0,999,097,611$ \\
\hline 84 & R-FCN & Resnet 101 & 300 & 20 & 23,8 & 39,7 & 16,8 & 2,1 & 25,2 & 40,1 & 6848 & 713 & 79 & 413 & 10 & 90 & 6 & 2714 & $0,998,732,677$ \\
\hline 85 & R-FCN & Resnet 101 & 300 & 10 & 22,1 & 37,6 & 14,4 & 1,8 & 23,9 & 36,3 & 6848 & 708 & 25 & 428 & 31 & 89 & 6 & 2714 & $0,995,053,005$ \\
\hline 86 & R-FCN & Resnet 101 & 600 & 300 & 30,4 & 43,3 & 28,3 & 7,2 & 32 & 51,4 & 6848 & 2878 & 75 & 1536 & 42 & 161 & 8 & 1073 & $0,998,702,076$ \\
\hline 87 & R-FCN & Resnet 101 & 600 & 100 & 30,5 & 43,4 & 28,5 & 7,4 & 32,1 & 51,6 & 6848 & 2651 & 19 & 1465 & 62 & 141 & 9 & 1073 & $0,999,719,894$ \\
\hline 88 & R-FCN & Resnet 101 & 600 & 50 & 30,1 & 43,3 & 27,9 & 7,3 & 31,8 & 50,4 & 6848 & 2591 & 100 & 1419 & 40 & 134 & 8 & 1073 & $0,995,150,449$ \\
\hline 89 & R-FCN & Resnet 101 & 600 & 20 & 28,3 & 41,3 & 25,7 & 6,2 & 30,4 & 46,5 & 6848 & 2600 & 69 & 1503 & 103 & 131 & 8 & 1073 & $0,965,018,477$ \\
\hline 90 & R-FCN & Resnet 101 & 600 & 10 & 25,3 & 37,9 & 21,9 & 4,9 & 27,7 & 40,5 & 6848 & 2530 & 34 & 1438 & 64 & 131 & 8 & 1073 & $0,926,073,406$ \\
\hline 91 & R-FCN & Resnet 101 & 300 & 300 & 27,6 & 43,3 & 22,7 & 4,4 & 29 & 46,1 & 6848 & 2190 & 26 & 1330 & 25 & 147 & 7 & 10,067 & $0,993,167,256$ \\
\hline 92 & R-FCN & Resnet 101 & 300 & 100 & 27,4 & 43,5 & 22,3 & 4,2 & 28,9 & 45,7 & 6848 & 2101 & 97 & 1314 & 21 & 133 & 6 & 10,067 & $0,928,019,446$ \\
\hline 93 & R-FCN & Resnet 101 & 300 & 50 & 27 & 43,3 & 21,6 & 3,7 & 28,7 & 44,5 & 6848 & 2072 & 86 & 1325 & 57 & 128 & 6 & 10,067 & $0,897,098,041$ \\
\hline 94 & R-FCN & Resnet 101 & 300 & 20 & 25,2 & 42 & 18,6 & 3 & 27,4 & 40,7 & 6848 & 2088 & 86 & 1303 & 23 & 127 & 6 & 10,067 & $0,871,237,262$ \\
\hline 95 & R-FCN & Resnet 101 & 300 & 10 & 23,1 & 39,4 & 15,7 & 2,4 & 25,5 & 36,5 & 6848 & 1943 & 93 & 1326 & 57 & 126 & 6 & 10,067 & $0,800,720,967$ \\
\hline 96 & R-FCN & Resnet 101 & 600 & 300 & 31,9 & 43 & 30,8 & 10,4 & 33,7 & 52,4 & 6848 & 7746 & 394 & 4823 & 145 & 389 & 14 & 39,178 & $0,772,787,428$ \\
\hline 97 & R-FCN & Resnet 101 & 600 & 100 & 31,7 & 43,2 & 30,7 & 10 & 33,8 & 52,2 & 6848 & 7143 & 97 & 4747 & 125 & 327 & 13 & 39,178 & $0,847,051,503$ \\
\hline 98 & R-FCN & Resnet 101 & 600 & 50 & 31,1 & 42,9 & 30,1 & 9,3 & 33,3 & 50,8 & 6848 & 7842 & 163 & 4769 & 108 & 313 & 12 & 39,178 & $0,779,429,498$ \\
\hline 99 & R-FCN & Resnet 101 & 600 & 20 & 28,8 & 41,7 & 26,9 & 7,2 & 31,5 & 46,1 & 6848 & 7562 & 251 & 4801 & 134 & 304 & 13 & 39,178 & $0,617,586,292$ \\
\hline 100 & R-FCN & Resnet 101 & 600 & 10 & 25,6 & 38,8 & 22,6 & 5,4 & 28,3 & 40,1 & 6848 & 7553 & 244 & 4685 & 113 & 302 & 13 & 39,178 & $051,970,279$ \\
\hline 101 & R-FCN & Inception V2 & 300 & 300 & 15,4 & 27,8 & 7 & 0 & 14,5 & 29,5 & 1806 & 286 & 14 & 144 & 7 & 72 & 5 & 5,09 & 1 \\
\hline 102 & $\mathrm{R}-\mathrm{FCN}$ & Inception V2 & 300 & 100 & 15,5 & 27,9 & 7,2 & 1 & 14,6 & 29,9 & 1806 & 208 & 11 & 125 & 6 & 69 & 6 & 5,09 & $0,999,535,091$ \\
\hline 103 & R-FCN & Inception V2 & 300 & 50 & 15,3 & 27,7 & 7,1 & 1 & 14,5 & 29,6 & 1806 & 202 & 13 & 125 & 6 & 66 & 6 & 5,09 & $0,998,471,992$ \\
\hline
\end{tabular}




\begin{tabular}{|c|c|c|c|c|c|c|c|c|c|c|c|c|c|c|c|c|c|c|c|}
\hline & Architecture & Extractor & Resolution & $\begin{array}{l}\# \\
\text { Proposals }\end{array}$ & $\mathrm{mAP}$ & $\begin{array}{l}\mathrm{mAP} \\
\text { (large) }\end{array}$ & $\begin{array}{l}\text { mAP } \\
\text { (medium) }\end{array}$ & $\begin{array}{l}\mathrm{mAP} \\
\text { (small) }\end{array}$ & $\begin{array}{l}\text { mAP @. } \\
75 I O U\end{array}$ & $\begin{array}{l}\text { mAP @. } \\
50 I O U\end{array}$ & $\begin{array}{l}\text { \# model } \\
\text { params }\end{array}$ & Memory & $\begin{array}{l}\text { Memory } \\
\text { std }\end{array}$ & $\mathrm{CPU}$ & $\begin{array}{l}\text { CPU } \\
\text { std }\end{array}$ & GPU & $\begin{array}{l}\text { GPU } \\
\text { std }\end{array}$ & FLOPS & DEA result \\
\hline 104 & R-FCN & Inception V2 & 300 & 20 & 14,7 & 26,8 & 6,5 & 0 & 14,1 & 27,9 & 1806 & 177 & 10 & 123 & 8 & 64 & 6 & 5,09 & $0,999,428,009$ \\
\hline 105 & R-FCN & Inception V2 & 300 & 10 & 13,8 & 25,2 & 5,7 & 0 & 13,6 & 25,6 & 1806 & 164 & 12 & 121 & 7 & 66 & 6 & 5,09 & $099,969,596$ \\
\hline 106 & R-FCN & Inception V2 & 600 & 300 & 19,8 & 31,8 & 15,2 & 1,8 & 18,2 & 38,3 & 1806 & 715 & 6 & 445 & 16 & 101 & 6 & 1985 & $0,997,671,385$ \\
\hline 107 & R-FCN & Inception V2 & 600 & 100 & 20,1 & 31,9 & 15,6 & 2 & 18,5 & 39,1 & 1806 & 647 & 19 & 394 & 15 & 87 & 6 & 1985 & $0,978,431,006$ \\
\hline 108 & R-FCN & Inception V2 & 600 & 50 & 19,7 & 31,6 & 15 & 2 & 18,3 & 38,1 & 1806 & 624 & 25 & 391 & 19 & 84 & 7 & 1985 & $0,961,580,826$ \\
\hline 109 & R-FCN & Inception V2 & 600 & 20 & 18,3 & 30,1 & 13,2 & 1,8 & 17,4 & 34,7 & 1806 & 609 & 9 & 378 & 5 & 81 & 6 & 1985 & $0,957,027,022$ \\
\hline 110 & R-FCN & Inception V2 & 600 & 10 & 15,9 & 26,5 & 10,6 & 1,6 & 15,6 & 29,5 & 1806 & 581 & 12 & 376 & 8 & 79 & 7 & 1985 & $0,890,098,397$ \\
\hline 111 & R-FCN & Inception Resnet V2 & 300 & 300 & 22,5 & 39,6 & 13,2 & 1 & 22,7 & 39,8 & 6506 & 696 & 108 & 339 & 10 & 108 & 6 & 1499 & 1 \\
\hline 112 & R-FCN & Inception Resnet V2 & 300 & 100 & 22,8 & 39,8 & 13,8 & 1,1 & 23 & 40,7 & 6506 & 642 & 71 & 315 & 13 & 100 & 7 & 1499 & $0,999,804,391$ \\
\hline 113 & R-FCN & Inception Resnet V2 & 300 & 50 & 22,8 & 39,6 & 14 & 1,2 & 23 & 40,6 & 6506 & 679 & 33 & 314 & 7 & 101 & 6 & 1499 & $0,999,906,686$ \\
\hline 114 & R-FCN & Inception Resnet V2 & 300 & 20 & 22,3 & 38,9 & 13,5 & 1 & 22,8 & 39,1 & 6506 & 611 & 42 & 312 & 13 & 99 & 6 & 1499 & $0,999,937,914$ \\
\hline 115 & R-FCN & Inception Resnet V2 & 300 & 10 & 21,1 & 37,5 & 12,1 & 1,1 & 22,1 & 36 & 6506 & 637 & 96 & 324 & 10 & 98 & 7 & 1499 & $0,997,406,826$ \\
\hline 116 & R-FCN & Inception Resnet V2 & 600 & 300 & 30,7 & 46,2 & 26,8 & 5,6 & 31,7 & 52,3 & 6506 & 2173 & 108 & 1048 & 24 & 165 & 6 & 6396 & $0,994,306,459$ \\
\hline 117 & R-FCN & Inception Resnet V2 & 600 & 100 & 30,9 & 46,4 & 27 & 6,1 & 31,9 & 52,8 & 6506 & 2121 & 36 & 993 & 26 & 150 & 6 & 6396 & $0,998,005,949$ \\
\hline 118 & R-FCN & Inception Resnet V2 & 600 & 50 & 30,7 & 46,1 & 26,8 & 6,2 & 32 & 52,3 & 6506 & 2150 & 83 & 1060 & 41 & 146 & 6 & 6396 & $0,987,753,468$ \\
\hline 119 & R-FCN & Inception Resnet V2 & 600 & 20 & 29,3 & 44,9 & 25 & 5,4 & 31,1 & 48,8 & 6506 & 2051 & 12 & 1007 & 30 & 144 & 6 & 6396 & $0,996,588,879$ \\
\hline 120 & R-FCN & Inception Resnet V2 & 600 & 10 & 27,1 & 42,7 & 22,1 & 4,4 & 29,3 & 43,9 & 6506 & 2151 & 62 & 1034 & 12 & 141 & 5 & 6396 & $0,949,050,948$ \\
\hline 121 & R-FCN & Inception Resnet V2 & 300 & 300 & 26 & 43,5 & 18,7 & 2 & 26,4 & 45,3 & 6506 & 1609 & 37 & 827 & 14 & 142 & 7 & 4774 & $0,964,248,588$ \\
\hline 122 & R-FCN & Inception Resnet V2 & 300 & 100 & 26 & 43,5 & 18,6 & 2,1 & 26,4 & 45,2 & 6506 & 1557 & 28 & 841 & 10 & 134 & 6 & 4774 & $0,943,934,531$ \\
\hline 123 & R-FCN & Inception Resnet V2 & 300 & 50 & 25,6 & 43,2 & 17,9 & 1,9 & 26,1 & 44,3 & 6506 & 1564 & 14 & 808 & 5 & 133 & 7 & 4774 & $0,973,978,512$ \\
\hline 124 & R-FCN & Inception Resnet V2 & 300 & 20 & 24,4 & 42,4 & 15,9 & 1,7 & 25,4 & 41,3 & 6506 & 1499 & 83 & 796 & 3 & 128 & 8 & 4774 & $0,892,191,711$ \\
\hline 125 & R-FCN & Inception Resnet V2 & 300 & 10 & 22,5 & 40,1 & 13,5 & 1,5 & 23,9 & 37,4 & 6506 & 1501 & 102 & 791 & 14 & 131 & 6 & 4774 & $0,805,923,613$ \\
\hline 126 & R-FCN & Inception Resnet V2 & 600 & 300 & 32,3 & 46,9 & 29,3 & 8 & 33,8 & 53,9 & 6506 & 7289 & 46 & 3220 & 60 & 368 & 8 & 20,545 & $094,355,266$ \\
\hline 127 & R-FCN & Inception Resnet V2 & 600 & 100 & 32,1 & 46,8 & 29,3 & 8 & 33,7 & 53,7 & 6506 & 6653 & 53 & 3319 & 70 & 328 & 6 & 20,545 & $0,850,951,265$ \\
\hline 128 & R-FCN & Inception Resnet V2 & 600 & 50 & 31,5 & 46,4 & 28,6 & 7,6 & 33,3 & 52,2 & 6506 & 6771 & 258 & 3315 & 32 & 313 & 7 & 20,545 & $0,805,416,577$ \\
\hline 129 & R-FCN & Inception Resnet V2 & 600 & 20 & 29,7 & 45,2 & 26,4 & 6,7 & 32 & 48,3 & 6506 & 6958 & 137 & 3318 & 100 & 302 & 7 & 20,545 & $0,642,626,419$ \\
\hline 130 & R-FCN & Inception Resnet V2 & 600 & 10 & 27,1 & 42,7 & 22,7 & 4,8 & 29,7 & 42,9 & 6506 & 7001 & 40 & 3320 & 21 & 299 & 7 & 20,545 & $0,728,464,914$ \\
\hline 131 & R-FCN & MobileNet & 300 & 300 & 15 & 26,3 & 7,6 & 0 & 13,7 & 29,4 & 10,8 & 246 & 4 & 114 & 4 & 66 & 5 & 2,44 & 1 \\
\hline 132 & R-FCN & MobileNet & 300 & 100 & 15,2 & 26,5 & 7,9 & 1 & 13,8 & 30 & 10,8 & 170 & 10 & 94 & 8 & 57 & 7 & 2,44 & $0,999,939,609$ \\
\hline 133 & R-FCN & MobileNet & 300 & 50 & 15,2 & 26,5 & 7,8 & 1 & 13,8 & 30 & 10,8 & 159 & 10 & 92 & 8 & 60 & 5 & 2,44 & $099,971,832$ \\
\hline 134 & R-FCN & MobileNet & 300 & 20 & 14,7 & 25,9 & 7,3 & 1 & 13,6 & 28,7 & 10,8 & 150 & 10 & 96 & 7 & 59 & 5 & 2,44 & $0,999,863,575$ \\
\hline 135 & R-FCN & MobileNet & 300 & 10 & 13,8 & 24,4 & 6,5 & 1 & 13,1 & 26,4 & 10,8 & 137 & 11 & 97 & 5 & 58 & 5 & 2,44 & $0,999,592,874$ \\
\hline 136 & R-FCN & MobileNet & 600 & 300 & 16,5 & 25,8 & 13,2 & 1,5 & 14 & 33,8 & 10,8 & 607 & 17 & 341 & 25 & 86 & 5 & 9,49 & $0,996,511,331$ \\
\hline 137 & R-FCN & MobileNet & 600 & 100 & 17 & 26,2 & 13,8 & 1,8 & 14,3 & 35,1 & 10,8 & 537 & 6 & 296 & 8 & 73 & 6 & 9,49 & $0,992,550,738$ \\
\hline 138 & R-FCN & MobileNet & 600 & 50 & 16,9 & 26 & 13,7 & 2 & 14,2 & 34,8 & 10,8 & 514 & 2 & 283 & 8 & 70 & 6 & 9,49 & $0,994,425,298$ \\
\hline 139 & R-FCN & MobileNet & 600 & 20 & 15,9 & 24,9 & 12,6 & 1,8 & 13,7 & 32,3 & 10,8 & 511 & 15 & 285 & 8 & 69 & 5 & 9,49 & $0,969,886,745$ \\
\hline 140 & R-FCN & MobileNet & 600 & 10 & 14,1 & 22,2 & 10,6 & 1,3 & 12,5 & 27,9 & 10,8 & 507 & 20 & 280 & 9 & 67 & 5 & 9,49 & 0,920353119 \\
\hline
\end{tabular}




\section{References}

[1] J. Huang, V. Rathod, C. Sun, M. Zhu, A. Korattikara, A. Fathi, I. Fischer, Z. Wojna, Y. Song, S. Guadarrama, et al., Speed/accuracy trade-offs for modern convolutional object detectors, in: Proceedings of the IEEE Conference on Computer Vision and Pattern Recognition, 2017, pp. 7310-7311

[2] W. Liu, Z. Wang, X. Liu, N. Zeng, Y. Liu, F.E. Alsaadi, A survey of deep neural network architectures and their applications, Neurocomputing 234 (2017) 1126, doi:10.1016/j.neucom.2016.12.038.

[3] D. Wu, S.-J. Zheng, X.-P. Zhang, C.-A. Yuan, F. Cheng, Y. Zhao, Y.-J. Lin, Z.Q. Zhao, Y.-L. Jiang, D.-S. Huang, Deep learning-based methods for person reidentification: a comprehensive review, Neurocomputing 337 (2019) 354-371, doi:10.1016/j.neucom.2019.01.079.

[4] Y. Yuan, G. Xun, Q. Suo, K. Jia, A. Zhang, Wave2vec: deep representation learning for clinical temporal data, Neurocomputing 324 (2019) 31-42. Deep Learning for Biological/Clinical Data, doi: 10.1016/j.neucom.2018.03.074.

[5] H. Cuayuitl, A data-efficient deep learning approach for deployable multimodal social robots, Neurocomputing (2019), doi:10.1016/j.neucom.2018.09.104.

[6] X.W. Gao, C. James-Reynolds, E. Currie, Analysis of tuberculosis severity levels from ct pulmonary images based on enhanced residual deep learning architecture, Neurocomputing (2019), doi:10.1016/j.neucom.2018.12.086.

[7] J. Deng, W. Dong, R. Socher, L.-J. Li, K. Li, L. Fei-Fei, Imagenet: A large-scale hierarchical image database, in: Proceedings of the IEEE Conference on Computer Vision and Pattern Recognition, 2009. CVPR 2009, IEEE, 2009, pp. 248-255.

[8] M. Everingham, L. Van Gool, C.K.I. Williams, J. Winn, A. Zisserman, The pascal visual object classes (VOC) challenge, Int. J. Comput. Vis. 88 (2) (2010) 303338, doi:10.1007/s11263-009-0275-4.

[9] T.Y. Lin, M. Maire, S. Belongie, J. Hays, P. Perona, D. Ramanan, P. Dollár, C.L. Zitnick, Microsoft COCO: common objects in context, in: Lecture Notes in Computer Science (including subseries Lecture Notes in Artificial Intelligence and Lecture Notes in Bioinformatics), in: LNCS, 8693, 2014, pp. 740-755, doi:10.1007/978-3-319-10602-1_48.

[10] A. Charnes, W.W. Cooper, E. Rhodes, Measuring the efficiency of decision making units, Eur. J. Oper. Res. 2 (6) (1978) 429-444.

[11] A. Fernandez-Montes, F. Velasco, J. Ortega, Evaluating decision-making performance in a grid-computing environment using dea, Expert Syst. Appl. 39 (15) (2012) 12061-12070.

[12] D. Fernández-Cerero, A. Fernández-Montes, F. Velasco, Productive efficiency of energy-aware data centers, Energies 11 (8) (2018), doi:10.3390/en11082053.

[13] A. Amirteimoori, A. Emrouznejad, Optimal input/output reduction in production processes, Decis Support Syst. 52 (3) (2012) 742-747.

[14] C. Kao, S.-N. Hwang, Efficiency measurement for network systems: it impact on firm performance, Decis Support Syst. 48 (3) (2010) 437-446.

[15] H. Eilat, B. Golany, A. Shtub, R\&d project evaluation: an integrated dea and balanced scorecard approach, Omega (Westport) 36 (5) (2008) 895-912.

[16] A. Emrouznejad, B.R. Parker, G. Tavares, Evaluation of research in efficiency and productivity: a survey and analysis of the first 30 years of scholarly literature in dea, Socioecon. Plann. Sci. 42 (3) (2008) 151-157.

[17] A. Expósito-García, F. Velasco-Morente, How efficient are universities at publishing research? a data envelopment analysis of spanish state universities, El profesional de la información 27 (5) (2018) 1108-1115.

[18] A. Afonso, L. Schuknecht, V. Tanzi, Public sector efficiency: evidence for new eu member states and emerging markets, Appl. Econ. 42 (17) (2010) 2147-2164.

[19] M.R. González-Rodríguez, F. Velasco-Morente, L. González-Abril, La eficiencia del sistema de protección social español en la reducción de la pobreza, Papeles de población 16 (64) (2010) 123-154.

[20] M. Campos, A. Fernandez-Montes, J. Gavilan, F. Velasco, Public resource usage in health systems: a data envelopment analysis of the efficiency of health systems of autonomous communities in spain, Public Health 138 (2016) $33-40$

[21] J. Fernández-Serrano, V. Berbegal, F. Velasco, A. Expósito, Efficient entrepreneurial culture: a cross-country analysis of developed countries, Int. Entrepr. Manag. J. 14 (1) (2018) 105-127.

[22] A. Exposito, F. Velasco, Municipal solid-waste recycling market and the european 2020 horizon strategy: a regional efficiency analysis in spain, J. Clean Prod. 172 (2018) 938-948.

[23] M.T. Sanz-Díaz, F. Velasco-Morente, R. Yñiguez, E. Díaz-Calleja, An analysis of spain's global and environmental efficiency from a european union perspective, Energy Policy 104 (2017) 183-193.

[24] J.-J. Moreno-Moreno, F.V. Morente, M.T.S. Díaz, Assessment of the operationa and environmental efficiency of agriculture in latin america and the caribbean, Agricult. Econ. 64 (2) (2018) 74-88.

[25] A. Expósito, J. Fernández-Serrano, F. Velasco, Crecimiento económico, pobreza y desigualdad: un análisis de eficiencia para américa latina en el siglo xxi, Revista de Economía Mundial (47) (2017) 117-138.

[26] J.-J. Moreno-Moreno, T. Sanz-Diaz, F. Velasco-Morente, C. Ludena, A dea-based evaluation of latin america and the caribbean agricultural environmental performance under the assumption of natural and managerial efficiency, Revista de Economía Mundial (47) (2017) 157-178.
[27] A. Charnes, W.W. Cooper, E. Rhodes, Evaluating program and manageria efficiency: an application of data envelopment analysis to program follow through, Manage Sci. 27 (6) (1981) 668-697.

[28] R.D. Banker, A. Charnes, W.W. Cooper, Some models for estimating technical and scale inefficiencies in data envelopment analysis, Manage Sci 30 (9) (1984) 1078-1092.

[29] R. Barkhi, Y.-C. Kao, Evaluating decision making performance in the GDSS environment using data envelopment analysis, Decis. Support Syst. 49 (2) (2010) $162-174$.

[30] R. Ranjan, V.M. Patel, R. Chellappa, Hyperface: a deep multi-task learning framework for face detection, landmark localization, pose estimation, and gender recognition, IEEE Trans. Pattern Anal. Mach. Intell. 41 (1) (2019) 121-135.

[31] R. Olmos, S. Tabik, F. Herrera, Automatic handgun detection alarm in videos using deep learning, Neurocomputing 275 (2018) 66-72.

[32] Á. Arcos-García, J.A. Álvarez-García, L.M. Soria-Morillo, Deep neural network for traffic sign recognition systems: an analysis of spatial transformers and stochastic optimisation methods, Neural Netw. 99 (2018) 158-165.

[33] S. Ren, K. He, R. Girshick, J. Sun, Faster r-cnn: Towards real-time object detection with region proposal networks, in: Proceedings of the Advances in Neural Information Processing Systems, 2015, pp. 91-99.

[34] J. Dai, Y. Li, K. He, J. Sun, R-fen: Object detection via region-based fully convolutional networks, in: Proceedings of the Advances in Neural Information Processing Systems, 2016, pp. 379-387.

[35] W. Liu, D. Anguelov, D. Erhan, C. Szegedy, S. Reed, C.Y. Fu, A.C. Berg, SSD: Single shot multibox detector, in: Lecture Notes in Computer Science (including subseries Lecture Notes in Artificial Intelligence and Lecture Notes in Bioinformatics), in: LNCS, 9905, 2016, pp. 21-37, doi:10.1007/978-3-319-46448-0_2.

[36] J. Redmon, A. Farhadi, Yolo9000: Better, faster, stronger, in: Proceedings of the 2017 IEEE Conference on Computer Vision and Pattern Recognition (CVPR), 2017, pp. 6517-6525, doi:10.1109/CVPR.2017.690.

[37] A. Arcos-Garcia, J.A. Álvarez-García, L.M. Soria-Morillo, Evaluation of deep neural networks for traffic sign detection systems, Neurocomputing 316 (2018) 332-344.

[38] K. Simonyan, A. Zisserman, Very deep convolutional networks for large-scale image recognition, arXiv:1409.1556 (2014).

[39] K. He, X. Zhang, S. Ren, J. Sun, Deep residual learning for image recognition, in Proceedings of the IEEE Conference on Computer Vision and Pattern Recognition, 2016, pp. 770-778.

[40] S. Ioffe, C. Szegedy, Batch normalization: accelerating deep network training by reducing internal covariate shift, arXiv:1502.03167 (2015).

[41] C. Szegedy, V. Vanhoucke, S. Ioffe, J. Shlens, Z. Wojna, Rethinking the inception architecture for computer vision, in: Proceedings of the IEEE conference on Computer Vision and pattern Recognition, 2016, pp. 2818-2826.

[42] C. Szegedy, S. Ioffe, V. Vanhoucke, A.A. Alemi, Inception-v4, inception-resnet and the impact of residual connections on learning., in: Proceeding of the AAAI, 2017, pp. 4278-4284.

[43] A.G. Howard, M. Zhu, B. Chen, D. Kalenichenko, W. Wang, T. Weyand, M. Andreetto, H. Adam, Mobilenets: efficient convolutional neural networks for mobile vision applications, arXiv:1704.04861 (2017)

[44] O.B. Olesen, N.C. Petersen, Stochastic data envelopment analysis a review, Eur J. Oper. Res. 251 (1) (2016) 2-21

[45] A. Charnes, W.W. Cooper, Chance-constrained programming, Manage Sci. 6 (1) (1959) $73-79$

[46] K.C. Land, C.K. Lovell, S. Thore, Chance-constrained data envelopment analysis Manag. Decision Econ. 14 (6) (1993) 541-554.

[47] T.-Y. Lin, P. Dollár, R. Girshick, K. He, B. Hariharan, S. Belongie, Feature pyramid networks for object detection, in: Proceedings of the IEEE Conference on Computer Vision and Pattern Recognition, 2017, pp. 2117-2125.

[48] K. He, G. Gkioxari, P. Dollár, R. Girshick, Mask r-cnn, in: Proceedings of the IEEE International Conference on Computer Vision, 2017, pp. 2961-2969.

[49] M. Najibi, M. Rastegari, L.S. Davis, G-cnn: an iterative grid based object detector, in: Proceedings of the IEEE Conference on Computer Vision and Pattern recognition, 2016, pp. 2369-2377.

[50] T. Sueyoshi, M. Goto, Dea radial measurement for environmental assessment: a comparative study between japanese chemical and pharmaceutical firms, Appl. Energy 115 (2014) 502-513.

[51] Z. Huang, S.X. Li, Stochastic dea models with different types of input-output disturbances, J. Product. Anal. 15 (2) (2001) 95-113.

[52] A. Charnes, W.W. Cooper, Management models and industrial applications of linear programming, Manage. Sci. 4 (1) (1957) 38-91.

[53] A.E. Gelfand, A.F. Smith, Sampling-based approaches to calculating marginal densities, J. Am. Stat. Assoc. 85 (410) (1990) 398-409.

[54] M.-H. Chen, O.-M. Shao, J.G. Ibrahim, Monte Carlo Methods in Bayesian Computation, Springer Science \& Business Media, 2012.

[55] F.J. Ortega, J.M. Gavilan, Bayesian estimation of the half-normal regression model with deterministic frontier, Comput. Stat. 31 (3) (2016) 1059-1078.

[56] G.E. Box, G.C. Tiao, Bayesian Inference in Statistical Analysis, Addison-Wesley, 1973. Reading, Massachussetts

[57] F.J. Ortega, J. Basulto, A generalization of Jeffreys' rule for non regular models, Commun. Stat. Theory Methods 45 (15) (2016) 4433-4444. 